\title{
Design of an Efficient Controller for Arterial Oxygen Saturation in Neonatal Infants
}

A Dissertation
Presented to
the Faculty of the Graduate School
at the University of Missouri - Columbia
In Partial Fulfillment
Dr. Roger Fales, Dissertation Supervisor
of the Requirement for the Degree
Doctor of Philosophy

December 2013 
The undersigned, appointed by the dean of the Graduate School, have examined the dissertation entitled

\section{Design of an Efficient Controller for Arterial Oxygen Saturation in}

\section{Neonatal Infants}

presented by Lafta Alkurawy

a candidate for the degree of doctor of philosophy

and herby certify that, in their opinion, it is worthy of acceptance

Professor Roger Fales

Professor Naz Islam

Professor Alina Zare

Professor Sherif El-Gizawy 


\section{ACKNOWLEDGMENTS}

I first would like to thank Dr. Roger Fales for becoming my advisor during my study and he agreed to my advisor in spite he is out of the faculty of my department. He taught me the how to conduct research and aided in my development as a student. Throughout the years of research that I have known him, he has been the first person a great wealth in my life.

I would also like to thank Dr. Naz Islam for his guidance and advices during my Ph.D studies.

I would also like to thank Dr. Alina Zare for her advice to me during my Ph.D studies.

I would also like to thank Dr. Sherif El-Gizawy for his advice to me during my Ph.D studies.

Finally, I would like to acknowledge my wife and my children. My wife has provided me with unending support over the years of my study. Without her helping to me, I couldn't achieve all that I have during this time of my life. 


\section{Table of Contents}

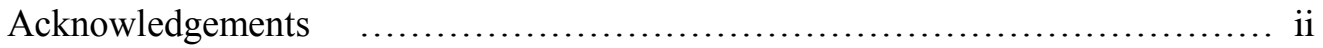

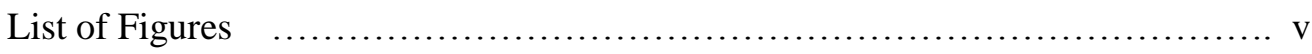

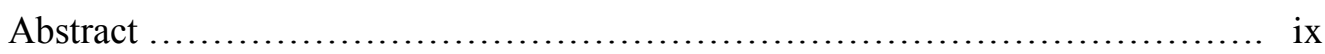

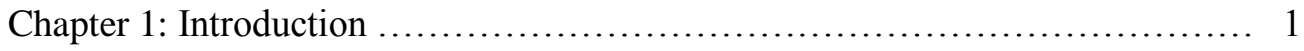

1.1. Background, Motivation, and Objectives ........................ 1

1.2. Literature Review ............................................ 5

1.2.1. Review of Respiratory System Model $\ldots \ldots \ldots \ldots \ldots \ldots \ldots \ldots \ldots \ldots \ldots \ldots$

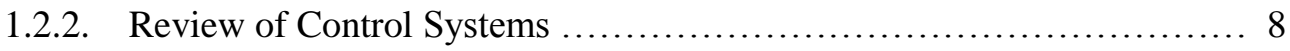

1.2.3. Review of PI Controllers ...................................... 13

1.2.4. Review of PID Controllers .................................... 14

1.2.5. Review of Model Predictive Control (MPC) ...................... 15

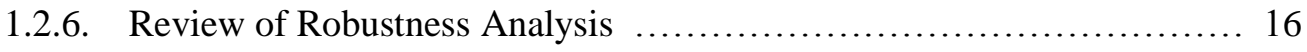

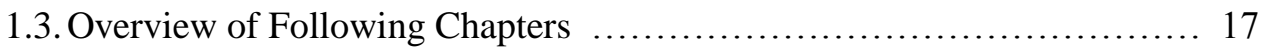

Chapter 2: Methodology: Modeling the Respiratory System Model ............. 19

2.1. Overview of the Respiratory System Model

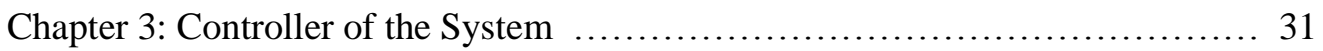

3.1. Proportional Integral (PI) Controller. ........................... 31

3.2. Proportional Integral Derivative (PID) Controller. .................. 39

3.3. Model Predictive Control (MPC). .............................. 47 
Chapter 4: Robustness

4.1. Robust Control Orientation Modelling. 65

4.1.1 Robust Control Design 67

4.2. Robust Control with MPC Controller. 75

4.2.1 Recursive Least Square (RLS) .75

4.2.2 Robustness with Model Predictive Control. 78

Chapter 5 Illustration of prototype 84

5.1 Overview of Device 84

Chapter 6: Conclusion and Signification 88

6.1. Conclusion of Data 88

Appendix A: Nomenclature 93

References 98

Vita 103 


\section{List of Figures}

Figure 1. Diagram of the respiratory control device

Figure 2. The System diagram of the three compartment lung model

Figure 3. The Graph of the oxygen dissociation curve

Figure 4. Graph showing the derivative of the oxygen dissociation curve

Figure 5. The block diagram of time constant $\tau$

Figure 6. Block diagram of gain, $G_{p}$

Figure 7. The block diagram of $\Delta \mathrm{SpO}_{2}$

Figure 8. Output $\mathrm{SpO} 2$ from the linear system models when supplying a $0.1 \%$ FiO2 step input

Figure 9. The Block diagram of PI Controller

Figure 10. $\mathbf{S p O 2}$ with $\mathrm{Kp}=\mathbf{0 . 5}$ and $\mathbf{K i}=5$

Figure 11. $\mathbf{S p O 2}$ with $\mathbf{K p}=\mathbf{0 . 0 5}$ and $\mathbf{K i}=\mathbf{1 0}$

Figure 12. with $\mathbf{K p}=\mathbf{0 . 0 0 0 5}$ and $\mathbf{K i}=\mathbf{2 0}$

Figure 13. with $\mathbf{K p}=\mathbf{0 . 0 0 0 5}$ and $\mathbf{K i}=\mathbf{2 5}$

Figure 14. with $\mathbf{K p}=\mathbf{0 . 0 0 0 5}$ and $\mathbf{K i}=\mathbf{3 0}$

Figure 15. with $\mathbf{K p}=\mathbf{0 . 0 0 0 5}$ and $\mathbf{K i}=\mathbf{4 0}$

Figure 16. with $\mathbf{K p}=\mathbf{0 . 0 0 0 5}$ and $\mathbf{K i}=\mathbf{5 0}$ 
Figure 17. with $\mathbf{K p}=\mathbf{0 . 0 5}$ and $\mathbf{K i}=\mathbf{5 5}$

Figure 18. with $\mathbf{K p}=\mathbf{0 . 0 5}$ and $\mathbf{K i}=\mathbf{1 0}$

Figure 19. with $\mathbf{K p}=\mathbf{0 . 0 5}$ and $\mathbf{K i}=\mathbf{2 . 2}$

Figure 20. The block diagram of PID controller

Figure 21. $\mathbf{S p O 2}$ with $\mathbf{K p}=\mathbf{0 . 1}, \mathbf{K i}=\mathbf{1 0 0}$, and $\mathbf{K d}=\mathbf{0 . 0 0 0 0 1}$

Figure 22. $\mathbf{S p O 2}$ with $\mathrm{Kp}=\mathbf{0 .} \mathbf{1}, \mathbf{K i}=\mathbf{5 0}$ and $\mathrm{Kd}=\mathbf{0 . 0 0 0 0 1}$

Figure 23. $\mathbf{S p O 2}$ with $\mathrm{Kp}=\mathbf{0 . 0 0 0 1}, \mathbf{K i}=\mathbf{1 0 0 0}$, and $\mathrm{Kd}=\mathbf{0 . 0 0 0 0 1}$

Figure 24. $\mathbf{S p O 2}$ with $\mathbf{K p}=\mathbf{0 . 0 0 0 0 1}, \mathbf{K i}=\mathbf{2 0 0 0}$, and $\mathbf{K d}=\mathbf{0 . 0 0 0 0 1}$

Figure 25. SpO2 with $\mathbf{K p}=\mathbf{0 . 0 0 0 0 1}, \mathbf{K i}=\mathbf{3 0 0 0}$, and $\mathbf{K d}=\mathbf{0 . 0 0 0 0 1}$

Figure 26. $\mathbf{S p O 2}$ with $\mathrm{Kp}=\mathbf{0 . 0 0 0 0 1}, \mathbf{K i}=\mathbf{4 0 0 0}$, and $\mathrm{Kd}=\mathbf{0 . 0 0 0 0 1}$

Figure 27. $\mathbf{S p O 2}$ with $\mathbf{K p}=\mathbf{0 . 0 0 0 0 1}, \mathbf{K i}=\mathbf{4 5 0 0}$, and $\mathbf{K d}=\mathbf{0 . 0 0 0 0 1}$

Figure 28. $\mathbf{S p O 2}$ with $\mathbf{K p}=\mathbf{0 . 0 0 0 0 1}, \mathbf{K i}=\mathbf{5 0 0 0}$, and $\mathbf{K d}=\mathbf{0 . 0 0 0 0 1}$

Figure 29. $\mathrm{SpO2}$ with $\mathrm{Kp}=\mathbf{0 . 1}, \mathrm{Ki}=\mathbf{1 0 0 0}$, and $\mathrm{Kd}=\mathbf{0 . 0 0 0 0 1}$

Figure 30. $\mathbf{S p O 2}$ with $\mathrm{Kp}=\mathbf{0 . 0 0 1}, \mathrm{Ki}=\mathbf{1 0 0 0}$, and $\mathrm{Kd}=\mathbf{0 . 0 0 1}$

Figure 31. $\mathrm{SpO2}$ with $\mathrm{Kp}=\mathbf{0 . 1}, \mathbf{K i}=\mathbf{3 0 0}$, and $\mathrm{Kd}=\mathbf{0 . 0 0 0 1}$

Figure 32. The Basic Structure of MPC

Figure 33. Receding Horizon strategy 
Figure 34. Output $\mathrm{SpO} 2$ with manipulated variable at $\Gamma_{y}=\mathbf{1}, \Gamma_{u}=\mathbf{1 0}$

Figure 35. Output $\mathrm{SpO} 2$ with manipulated variable at $\Gamma_{y}=1, \Gamma_{u}=20$

Figure 36. Output SpO2 with manipulated variable at $\Gamma_{y}=\mathbf{1}, \Gamma_{u}=\mathbf{5}$

Figure 37. Output $\mathrm{SpO2}$ with manipulated variable at $\Gamma_{y}=\mathbf{1}, \Gamma_{u}=2$

Figure 38. (a) Multiplicative uncertainty transfer function bounding the maximum error for the set parameter range

(b) Bode plot for transfer function of $w_{I}$.

Figure 39. Diagram of robust control model with multiplicative uncertainty.

Figure 40. Bode diagram of the $w_{P}$ performance weight.

Figure 41. Block diagram of the $\mathbf{P}$ matrix structure.

Figure 42. Block diagram of the $N-\Delta$ configuration.

Figure 43. The H-infinity norm of $\boldsymbol{N}_{22}$ is less than one for all frequencies.

Figure 44. The H-infinity norm of $N_{11}$ is less than one for all frequencies.

Figure 45. The maximum singular value and structured singular value of the $\mathrm{N}$ matrix is less than one for all frequencies.

Figure 46. The real control output with the identification by RLS.

Figure 47. Bode diagram of the $w_{P}$ performance weight.

Figure 48. The H-infinity norm of $\boldsymbol{N}_{22}$ is less than one for all frequencies 
Figure 49. The H-infinity norm of $\boldsymbol{N}_{\mathbf{1 1}}$ is less than one for all frequencies for the $\boldsymbol{\mu}$-synthesis controller .

Figure 50. Figure 35. The maximum singular value and structured singular value of the $\mathbf{N}$ matrix is less than one for all frequencies.

Figure 51. Block Diagram of the device and connections.

Figure 52. a) 24 V DC Motor with Encoder. b. Knob tuning device connected to the blend valve knob

Figure 53. The National Instruments sbRIO microcontroller and power supply. 


\section{Abstract}

A common problem for premature infants is respiratory distress syndrome (IRDS), also called neonatal respiratory distress syndrome, or respiratory distress syndrome of newborn. Due to IRDS, the infant requires intervention in the form of respiratory support to increase the inspired oxygen. Physicians must keep the range of the Arterial Oxygen Saturation $\left(\mathrm{SpO}_{2}\right)$ between $82-95 \%$ to help the premature infants to get oxygen enough while preventing other complications. If the blood oxygen saturation is more than $95 \%$ or less than $82 \%$, the infant is at risk for retinopathy of prematurity. The control is analyzed using PI, PID, Model Predictive Controller (MPC), Robust control wit PID and Robust control with MPC to ensure stability and minimum settling time to reach the accuracy of output $\mathrm{SpO}_{2}$ by applying the Fraction of Inspired Oxygen $\left(\mathrm{FiO}_{2}\right)$ as control action. MPC is an optimal control strategy based on numerical optimization by using a system model and optimizing at regular intervals. We can predict the future control inputs and future plant responses. An error model is created using the resulting ranges of system gains and time constant from [18]. The $\mu-$ synthesis controller is developed to control the oxygen percentage of inspired air and performance specifications are defined. The $H_{\infty}$ method is used to determine the robust stability and robust performance are achieved with the system uncertainty that described by the error model. A comparison among a static proportional integral, proportional integral derivative, the model predictive controller, the robust controller with PID controller, and the 
robust controller with MPC found that the robust controller with MPC displays the best performance for a system with large ranges of model parameters. 


\section{Chapter 1: Introduction}

\subsection{Background, Problem, and Objectives}

A wide spread problem for premature infants is respiratory distress syndrome (IRDS), also called neonatal respiratory distress syndrome, or respiratory distress syndrome of newborn, previously called hyaline membrane disease (HMD). IRDS is a syndrome in premature infants caused by developmental insufficiency of surfactant production and structural immaturity in the lungs. It can also result from a genetic problem with the production of surfactant associated proteins. IRDS affects about $1 \%$ of newborn infants and is the leading cause of death in preterm infants. The incidence decreases with advancing gestational age, from about $50 \%$ in babies born at $26-28$ weeks, to about $25 \%$ at $30-31$ weeks. The syndrome is more frequent in infants of diabetic mothers and in the second born of premature twins [1]. Respiratory distress syndrome (RDS) is a breathing disorder that affects newborns.

RDS rarely occurs in full-term infants. The disorder is more common in premature infants born about 6 weeks or more before their due dates. RDS is more common in premature infants because their lungs are not able to make enough surfactant. Surfactant is a liquid that coats the inside of the lungs. It helps keep them open so that infants can breathe in air once they are born. Without enough surfactant, the lungs collapse and the infant has to work hard to breathe. He or she might not be able to breathe in enough oxygen to support the body's 
organs. The lack of oxygen can damage the baby's brain and other organs if proper treatment isn't given [2]. Respiratory distress syndrome occurs in infants born prematurely and is a consequence of immature lung anatomy and physiology. In premature of stressed infants, atelectasis from the collapse of the terminal alveoli resulting from lack of surfactant appears after the first few hours of life. In premature infants, surfactant production is limited and stores are quickly depleted. Surfactant production may be further diminished by other unfavorable conditions such as high oxygen concentration, poor pulmonary drainage, or effects of respirator management [3].The arterial oxygen saturation $\left(\mathrm{SpO}_{2}\right)$ must be kept within a certain range which is usually $85-92 \%$. The clinics provided alarms to notify medical personal if the premature infant is outside of the range of safety of $\mathrm{SpO}_{2}$. If the $\mathrm{SpO}_{2}$ level is maintained above $92 \%$, a state of hypoxia could result in visual impairment or blindness. If the $\mathrm{SpO}_{2}$ level is maintained below $85 \%$, a state of hypoxia could result in tissue damage and brain injury.

Research has shown that the neonatal infants spend only $50 \%$ of the time within the acceptable ranges under manual control of the $\mathrm{FiO}_{2}$. The remaining $20 \%$ is spent below the acceptable $\mathrm{SpO}_{2}$ range and $30 \%$ above the acceptable $\mathrm{SpO}_{2}$ range. However it has been shown that the safety limits are often set outside the recommended ranges $[4,5]$. The $\mathrm{SpO}_{2}$ is measured using a noninvasive pulse oximeter and is regulated by increasing the fraction of inspired oxygen $\left(\mathrm{FiO}_{2}\right)$. The accuracy of pulse oximetry is limited when the readings decrease below $80 \%$, particularly in neonates with fetal hemoglobin. In adults, an $\mathrm{SpO}_{2}$ of $85 \%$ to $94 \%$ 
is associated with a $\mathrm{PaO}_{2}$ of 50 to $75 \mathrm{~mm} \mathrm{Hg}$. Comparable ranges of oxygen saturation measurements that account for fetal hemoglobin must be established for neonates [6].

The goal of this dissertation is to design a controller for the $\mathrm{FiO}_{2}$ to regulate the measured $\mathrm{SpO}_{2}$. It is very important to alleviate the workload of nurses in an intensive care unit when this controller is used to reduce the time and amount of harmful desaturation events. The controller depends on model predictive control (MPC) to control $\mathrm{FiO}_{2}$ to get the best value of $\mathrm{SpO}_{2}$. The main motive of MPC is to find the input signal that best corresponds to some criterion which predicts how the system will behave applying this signal. Model Predictive Control (MPC) is an optimal control strategy based on numerical optimization. By using a systems model and optimizing at regular intervals, we can predict the future control inputs and future plant responses. Several different controllers were designed and tested to see which performed the best. The controller selected an optimal $\mathrm{FiO}_{2}$ input to keep the infant at a safe range of $\mathrm{SpO}_{2}$. The controller also attempted to reject the effects the heart rate (HR) and respiratory rate (RR) have on the infants $\mathrm{SpO}_{2}$. MPC has been developed so that stability, optimality, and robustness properties are well defined. A diagram of the device in the clinical setting can be seen in Figure 1. 


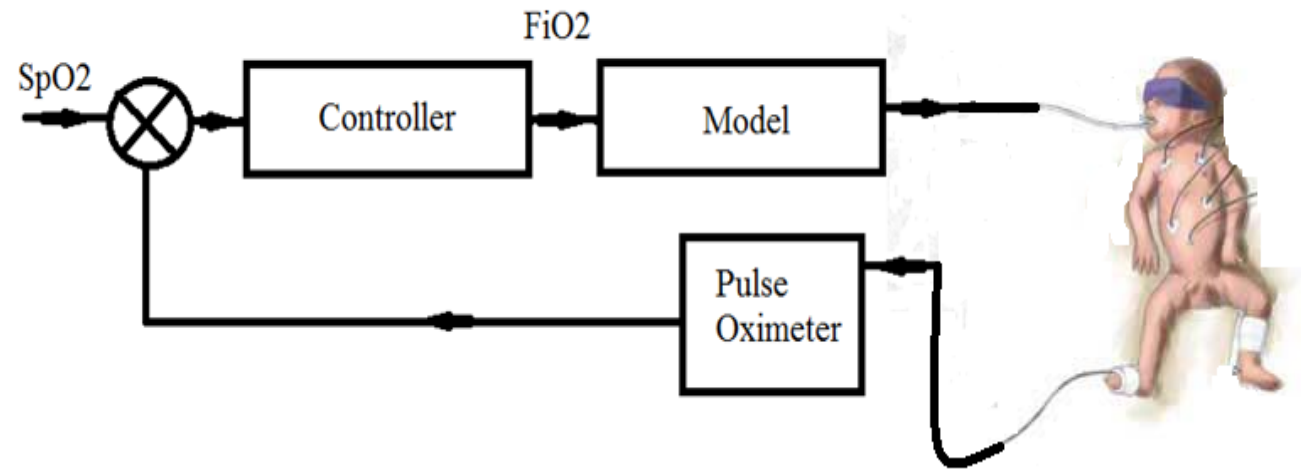

Figure 1. Diagram of the respiratory control device. 


\subsection{Literature Review}

\subsubsection{Review of Respiratory System Models}

The following researchers have developed models for the human respiratory system. The first formulation was made by L. Roa, and OrtegaMartinez J.I. (1997) [7]. They considered the two external processes included in the term respiratory system as the absorption of $\mathrm{O}_{2}$ and the removal of $\mathrm{CO}_{2}$ from the body and internal respiratory, the gaseous exchanges between the cells and their fluid mediums. Their mathematical model has been designed for the analysis of the response of the organism to different pathological situations. This paper explained how can transfer Oxygen $\left(\mathrm{O}_{2}\right)$ and Carbon Dioxide $\left(\mathrm{CO}_{2}\right)$ in compartments like Intracellular, Interstitial, Vascular and Alveolar.

Revow et al. [8] presented a model in 1989 which could successfully simulate the respiratory system of the newborn infant during the epoch of quiet sleep. The cerebrospinal fluid compartment in this model was not separated from the brain. This paper showed how we can analyze the lung compartment and the tissue compartment and how we can create equations for that.

Fleur T. Tehrani et al. [9] presented a mathematical model in 1993 which was used to study the effects of prematurity of peripheral chemo receptors on the respiratory function during the newborn period and to simulate the neonatal

respiratory control system. In this model, using a wide range of stimuli, the 
transient and steady state behavior were examined. This paper enabled us to find the parameters that we took in modeling.

S. Kanae, K. Murmatsu, Z.J. Yang and K. Wada et al. [10] presented a model in 2004 which is to fit each patient who is receiving artificial respiration treatment. They built a respiratory model of a lung that describes the dynamics of respiration. This model is a second - order nonlinear differential equation. They used a numerical integration technique; and a continuous - time identification model was derived. They used off- line and on-line parameters estimation algorithms and the model and the parameter estimation method was validated by clinical data.

Scott A. Sands, Bradley A. Edwards, and Venessa J. Kelly [11] presented a model analysis of the rate of arterial oxygen desaturation during apnea to demonstrate that pre-apneic ventilation, lung volume, cardiac output, hemoglobin content and blood volume exert unique effects on the rate of arterial oxygen desaturation throughout the time-course of desaturation, while metabolic oxygen consumption is uniformly influential throughout the process. They have provided a mathematical framework for quantifying the relative importance of key cardiorespiratory factors on the rate of arterial oxygen desaturation during apnea, with particular relevance to preterm infants.

C.L. Yu [12] presented a model that considered a linearization of the oxygen dissociation curve to change the partial pressure of oxygen in the artery to the oxygen saturation percent. This oxygen dissociation curve was the first 
proposed by Severinghaus, and it is used to convert partial pressure of oxygen to oxygen saturation in blood. This paper was very important for us to help us to create the modeling of the efficient controller for Arterial Saturation in Infants. They informed us above the ways to study the compartments work and how we can make the equation of modeling, how we can use the Oxygen Saturation curve and the limit of $\mathrm{SpO}_{2}$ with changing in values of $\mathrm{PaO}_{2}$.

We took these results in the creation of our modeling. 


\subsubsection{Review of Control Systems}

There are many control systems that have been presented in the literature. L. Zhang, and R.G. Cameron proposed a real - time rule-based control strategy for blood gas regulation of preterm infants under ventilation treatment [13]. The General Predictive Control (GPC) controller was investigated using computer simulation. They used a first - order autoregressive-moving-average (ARMA) model to represent the respiratory system and Recursive Least Square (RLS) estimation algorithm to cope with nonlinearity and time varying characteristics of the system. Based on the results of the simulation and support from experienced pediatritions, the scheme is very promising for clinical applications. They chose GPC to control the partial pressure $\left(P_{a O_{2}}\right)$ and $\left(P_{a C O_{2}}\right)$ by adjusting the concentration of oxygen $\left(F_{i} O_{2}\right)$ in the air they inspire. The results of the simulation were very encouraging from the expert system for a set of ventilator adjustments. From this paper we learned what the effect is of constraints on the GPC to make decisions for changing $F_{i} O_{2}$ levels.

In 1991 Tehrani et al. proposed a PID controller using a feedback signal of arterial oxygen saturation of the premature infant. It was used to adjust the concentration of inspired oxygen under the incubator [14]. They used a computer simulation, and the performance of the control system was evaluated under different test conditions to investigate the performance of the control system. The concentration of oxygen in the inspired gas $\left(F_{i} O_{2}\right)$ of the neonate was adjusted to provide for sufficient oxygenation of the blood and was low enough to prevent the damaging effects of oxygen toxicity. They calculated the values of parameters of 
a PID controller after a number of preliminary simulation experiments. The effect of the PID controller on the system is to make arterial pressure reach the set point with in a small time period. The results were stable and indicative of the effectiveness of the controller under two different tests.

In 1991 John Taube M.S. and Vinod Bhutani M.D. et al. proposed a computer simulation with PID controller between the oxygen sensing and an oxygen blender for premature infants in [15]. They used a closed loop oxygen controller for the automatic control of supplemental oxygen because the regulation in open loop is a mismatch between the supplemental oxygen provided and the needs of the patient. PID controller software program was used to calculate a signal to the control oxygen blender output by using hemoglobin saturation (HSAT) from a pulse oximeter as feedback. It produced a fast response of hemoglobin saturation with little overshoot and gave a desired steady state error. The automatic control of oxygen was a more accurate method of regulating the blood oxygen level in the premature infants.

C. Yu, W. He, J. So, R. Roy and H. Kaufman. et al proposed to use a multiple - model adaptive controller (MMAC) for regulating oxygen saturation with changing input $\mathrm{FiO}_{2}$ [16]. The procedure in MMAC assumes that the system can be represented by one of a finite number of models and used to desensitize the system to gain variation. Computer-based proportional - integral (PI) simulations demonstrated the effectiveness of the algorithm over a wide variation of plant parameters. The fixed PI controller was designed to give no steady state error and the simulation showed that variations in plant parameters did not adversely affect 
the transient response. The controller was commanded to raise $\mathrm{S}_{a} \mathrm{O}_{2}$ from an initial value of about $80 \%$ to a reference level of $95 \%$ and to maintain it at the new set point and by changing the values of gain, time constant and dead space time for plant at constant sampling period. Atypical step response illustration $\mathrm{S}_{a} \mathrm{O}_{2}$ changes, $\mathrm{F}_{i} \mathrm{O}_{2}$ level and the weights for each model. Results of both simulations and animal experiments demonstrate the ability of the MMAC controller to effectively regulate $\mathrm{S}_{a} \mathrm{O}_{2}$ despite the presence of system disturbances.

Paul E. Morozoff, Ron W. Evans and John A. Smyth et al proposed an automatic control to regulate blood oxygen saturation [17]. The automatic $S_{a} O_{2}$ controller was constructed to assist clinical staff in improving a premature infant's condition by reducing the duration and frequency of hypoxemic and hyperoxemic episodes. They used a control algorithm based on the sign of the error magnitude, velocity and acceleration as input and then applied these inputs to a state machine to determine the trend of the error. Error is defined as the observed oxygen saturation minus the target oxygen saturation. The feature of this algorithm was that it could accommodate the non-linearity of the system. Each of the state machines can provide $\mathrm{F}_{i} \mathrm{O}_{2}$ adjustment and delay times, and the state machine was built to identify trends of $\mathrm{S}_{a} \mathrm{O}_{2}$ moving towards or away from the target. A single set of machine parameters was used by the controller to regulate the oxygen saturation with eight infants in the clinical trials. During this study a generic set of state machine $F_{i} O_{2}$ increments, decrements and delay time was determined. They found that with large variability of physiology and $\mathrm{S}_{a} \mathrm{O}_{2}$ 
stability between neonates, a single set of state machine parameters could be used by the controller to regulate a patient's $\mathrm{S}_{a} \mathrm{O}_{2}$. They found that if $\mathrm{S}_{a} \mathrm{O}_{2}$ dropped suddenly as result of shunting, the controller could not react fast enough and that required the manual intervention as signaled by the controller's $\mathrm{S}_{a} \mathrm{O}_{2}$ limiting. Results from this paper proved that the automatic control systems are becoming more prevalent and increased the duration that the neonate spent at normal $\mathrm{S}_{a} \mathrm{O}_{2}$ and the number of manual interventions required by clinical staff.

Keim proposed to design single robust controller based on a linear model of premature infants [18]. The robust controller was designed based on an error model and performance specifications. He developed an adaptive controller based on estimated parameters and disturbances. The controller regulated the $\mathrm{FiO}_{2}$ while mitigating disturbances. The $H_{\infty}$ is used in control theory to synthesize controllers achieving robust performance or stabilization. The $H_{\infty}$ is used for plants having problems involving multivariable systems. In this paper, a performance requirement is developed in the frequency domain for the purpose of control design and analysis. To check for performance, the following inequality must hold for all frequencies,

$$
\left\|N_{22}\right\|_{\infty} \leq 1
$$

where $\left\|N_{22}\right\|_{\infty}$ is a frequency domain performance measure.

The plot of the $H_{\infty}$ norm of $N_{22}$ was always less than one, so the system has nominal performance. The control signals for adaptive and $H_{\infty}$ control systems have saturation limits such that the signals do not go below $0 \%$, since that level is 
considered to be equal to room air. The adaptive control system is able to reject the disturbances and has $0 \%$ overshoot. The $S_{p} O_{2}$ from the closed loop control simulation did not drop beneath $2 \%$ due to the disturbances. The robust control system has slow performance due to the low bandwidth frequency that is used for control design. The robust control system also has $0 \%$ overshoot. The control signal for the adaptive controller is smaller than that of the robust controller. For these reasons, the robust control is better than the adaptive control.

Deacha C., Anan W., and Kitiphol C. proposed to design an automatic control for oxygen intake via nasal cannula in premature infants [19]. They used a new computer - based system combining to the nasal cannula for automatically controlling the quantity of oxygen intake. A pulse oximeter is currently used in clinical settings for noninvasive and continuous monitoring of arterial oxygen in infants. In flow control of oxygen, commands are transferred from a computer into the data acquisition (DAC) interface by USB port. Then it sends digital data to drive a stepping motor for speed control. The performance of the system was evaluated by operating with a $\mathrm{SpO}_{2}$ simulator showing satisfactory results with low tracking error. The computer controlled the natal cannula $F_{i} O_{2}$ flow by using a pulse oximeter as indicator for arterial oxygen saturation in blood $\left(\mathrm{S}_{a} \mathrm{O}_{2}\right)$ in the feedback loop control, oxygen intake needed, calculated from the model is fed via controlled values. The process operates on a microcomputer programmed on the national Instruments $\operatorname{LabView}(\mathrm{R})$.

Nelson C., Tilo G., Ruth E., Gabriel M., Carmen H., and Edua proposed an algorithm for closed-loop inspired oxygen control for mechanical ventilation [20]. 
They developed an algorithm to maintain $\mathrm{SpO}_{2}$ within a target range. The closed - loop control was compared with continuous manual $\mathrm{FiO}_{2}$ adjustments by a nurse with a group of ventilated infants who presented with frequent episodes of hypoxemia. There were two $\mathrm{FiO}_{2}$ control modes : the $\mathrm{CFiO}_{2}$ algorithm defines

$\mathrm{SpO}_{2}$ ranges based on a user - defined target range of normal blood levels of oxygen (normoxemia); the $\mathrm{mFiO}_{2}$ was the reference mode to which the $\mathrm{CFiO}_{2}$ algorithm was compared consisted of manual adjustments of the $\mathrm{FiO}_{2}$ made continuously by a neonatal research nurse station, fully dedicated to maintain $\mathrm{SpO}_{2}$ within the same target range of (normoxemia) . Computerized analysis was used to calculate mean $\mathrm{SpO}_{2}$, frequency and duration of episodes of hypoxemia. They selected fourteen very low birth weight (VLBW) infants undergoing mechanical ventilation which were included in this study. Although it remains to be proven, they speculated that long-term closed - loop $\mathrm{FiO}_{2}$ control may reduce nursing time spent to maintain adequate oxygenation and reduce the risk of morbidity associated with supplemental oxygen.

\subsubsection{Review of PI Controllers}

Proportional Integral (PI) Controllers have been used in industry with linear and nonlinear systems. S. Anand, Aswin. V., and S. Rakesh kumar showed in 2011 a design continuously tuned adaptive PI controller for a non-linear process as a conical tank [21]. A simple tuning system was used to continuously tune the controller parameters in correspondence with the change in operating 
points. The tuning system had the ability to interpolate and extrapolate the relationship between the control variable and the controller parameters over entire span of control variables. Then the PI controller was able to produce minimum overshoots and minimum settling time. Rubiyah and Sigeru in 1994 used the PI controller to the temperature controlled water bath [22]. It has ability of the controllers to handle process with variable time delays. Tunyasrirut and Ngamwiwit in 1999 presented a design of adaptive PI controller to control the speed of separately excited DC motor by self - tuning [23]. The designed controller to control the armature voltage while the field voltage was fixed as a constant. F.T. Tehrani in 2001 designed control system was proposed for oxygen therapy for premature infants [24]. The control software is used as well as a PI control algorithm to provide fast and efficient response to changes in arterial oxygen saturation of the infant detected by pulse oximetery.

\subsubsection{Review of PID Controllers}

PID Control systems have been used with many industrial devices. Noor and Mahanijah in 2009 presented the comparison of performance between a PID temperature controller and a conventional on-off temperature controller for a home - applied refrigerator [25]. They designed PID and evaluated it using MATLAB Simulink software. They found that the proposed PID temperature controller performed better than the on-off controller in maintaining the set value 
of the system which is the inner temperature of the refrigerator and PID controller was working more efficiently to maintain the inner temperature of the refrigerator than the on-off controller. M.H. Moradi in 2003 presented to design of predictive PID controllers [26]. He proposed that a controller can deal with future set points and the process dead time can be incorporated without any need for approximation. He found that the main advantages of the proposed controller were that it can be used with systems of any order and the PID tuning can be used to adjust the controller performance. Arulmozhiyal and Kandiban in 2012 proposed an improved PID controller to control speed of brushes DC motor [27]. They presented simulation results of conventional PID controller and Fuzzy PID controller of the three brushless DC motor. They found that the Fuzzy controller showed better performance than PID controller at lower and high speeds. Taube and Pillutla in 1988 developed with another colleague for closed loop supplemental oxygen treatment of newborn [28]. They used PID control design for an adaptive control system to maintain blood oxygen levels at desired levels. This design was clearly usable in an intensive care nursery environment.

\subsubsection{Review of Model Predictive Controller (MPC)}

Model Predictive Control (MPC) has been used in the academic and industrial studies. Alicia and Alejandro in 2010 proposed two controls to manage the air supply of the fuel-cell system [29]. They improved transient responses and better fuel-cell efficiency in the case of the efficiency maximization objective. 
C. Yu and W. He proposed a computer - based proportional - integral (PI) controller has been developed to control arterial oxygen levels in mechanically ventilated animals [16]. They designed a multiple model adaptive control (MMPC) to desensitize the system to these gain variables and compared it with the PI controller.

\subsubsection{Review of Robustness Analysis}

Very little work has been done to analyze the robustness of controllers for an oxygen saturation control system. In 2001 Tehrani showed robustness by testing the control system for two different desaturation periods [23]. The model range of parameters for testing the robustness of the controller was not large enough and no techniques such as $\mathrm{H}$-infinity robustness analysis were used to show that the control systems guaranteed robust performance and stability. Keim proposed a controller to control arterial oxygen saturation in neonatal infants [18]. Krone also proposed a robust controller to reject the disturbances caused by variations in Heart Rate (HR) and Respiratory Rate (RR) to keep the $\mathrm{SpO}_{2}$ at given set point [30]. Keim developed a single robust controller based on a linear model. The robust controller was designed based on an error model and performance specifications. Keim developed an adaptive controller based on estimate parameters and disturbances. The controller attempted to regulate the $F_{i} O_{2}$ while mitigate the affect of the disturbances. Krone designed a robust controller with an average $\mathrm{SpO}_{2}$ of $6.623 \mathrm{e}-004 \%$ and a maximum $\mathrm{SpO}_{2}$ value of $0.0725 \%$. The 
$\mathrm{SpO}_{2}$ was normalized at $90 \%$ and the $\mathrm{F}_{i} \mathrm{O}_{2}$ was normalized at $21 \%$. The $\mathrm{SpO}_{2}$ and $F_{i} O_{2}$ values presented were the difference between the actual values and the nominal values.

\subsection{Overview of Following Chapters}

In Chapter 2, the respiratory system model by $\mathrm{Yu}$ will be used to analyze the model after desaturation periods. We have chosen this model because it has one input that is $\mathrm{FiO}_{2}$ and one output that is $\mathrm{SpO}_{2}$. This model was relinearized to find a linear model at the operating point. Data from Columbia Regional Hospital now known as the University of Missouri Women's and Children's Hospital will be discussed and used to compare and will use Yu's model. In Chapter 3, we designed PI, PID, and MPC for the systems. A digital PI and PID controller designed to control $\mathrm{FiO}_{2}$ to get the range values of $\mathrm{SpO}_{2}$ between $85 \%$ to $92 \%$ with minimum overshoot and zero steady state error. A model Predictive Control (MPC) was designed to predict $\mathrm{SpO}_{2}$ by finding the best values of control horizon and moving horizon. The most important part of strategy was obtaining the control law. With the control law found, the values of $\mathrm{FiO}_{2}$ were determined that control the plant to get good response without peak overshoot and zero error steady state. In Chapter 4, a robust analysis of the system is performed and a robust controller is developed. The range of system gains and time constants used in the analysis are taken from Krone's thesis [30]. It is shown that a single, 
static controller can guarantee robust performance for all the ranges of parameters. In Chapter 5, an overview of the construction of the whole system with designing controller of an oxygen control prototype is presented. We suggest conclusions and a plan for future work. 


\section{Chapter 2: Methodology: Modeling the Respiratory System Model}

\subsection{Overview of the Respiratory System Model}

We used model that is based on prior research completed by Yu. This model was a nonlinear model to describe the relationship between $\mathrm{SpO}_{2}$ with the input $\mathrm{FiO}_{2}$. In this investigation, we took modeling by $\mathrm{Yu}$ with the effect of heart rate (HR) and respiratory rate (RR) as disturbances.

There are two major parts in the respiratory system: the lungs and the circulating blood that transports the oxygen to the other part of the human body. In the lungs, there are three compartments. The first compartment of the lung with volume, $V_{A}$, is perfused with blood flow, $Q_{p}$, and is ventilated with a respiratory rate of $\dot{V}_{A}$. The second lung compartment corresponds to the dead zone in the lung. All dead zone in the lung is lumped into one parameter called the dead zone ratio, $x_{d}$. The ventilation to the first lung volume can be define as

$$
\dot{V}_{A}=\left(\dot{1}-x_{d}\right) \dot{V}_{I}
$$

where $\dot{V}_{I}$ is the total respiratory rate. The third lung compartment is perfused with blood. It introduces a shunt ratio, $y_{s}$. The ratio will affect how much blood flow will reach the first lung compartment by

$$
Q_{p}=\left(1-y_{s}\right) Q
$$


where $\mathrm{Q}$ is the total blood flow to the respiratory system. We took linear and nonlinear models with the assumption that all flow is constant and unidirectional. Figure 2 shows the three - compartment lung model.

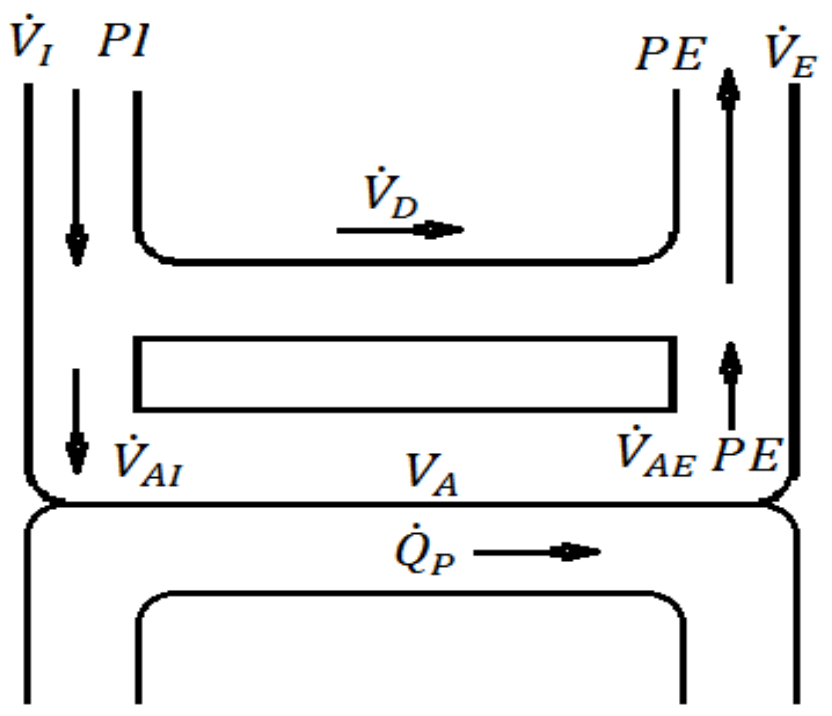

Figure 2. The System diagram of the three compartment lung model.

For the first lung compartment, the mass balance equation is derived as

$$
\begin{aligned}
& \dot{P}_{A} V_{A}=P_{I} \dot{V}_{A}-P_{A} \dot{V}_{A}+\frac{273+P T}{273}\left(\frac{760 \mathrm{mmHg}}{100 \text { vol\% }}\right) Q_{p}\left(C_{v}-C_{c}\right), \text { and } \\
& \dot{P}_{A} V_{A}=P_{I} \dot{V}_{A}-P_{A} \dot{V}_{A}+8.63 Q_{p}\left(C_{v}-C_{c}\right)
\end{aligned}
$$

where $V_{A}$ is the volume of the lung compartment where the alveoli are open and perfused with blood, $\dot{V}_{A}$ is the volume rate of change of inspiratory air to the lung sub compartment, $Q_{p}$ is the pulmonary capillary blood flow; $P_{A}$ is the alveolar oxygen partial pressure in the lung, $C_{c}$ is the oxygen content of pulmonary end capillary blood in vol, and $C_{v}$ is the oxygen content of mixed venous blood in vol\% [12]. $P T$ is the patient's temperature which is assumed to be $37^{\circ} \mathrm{C}$. The 
constant 8.63 is a factor to convert gas concentrations and saturated and saturated water vapor conditions to temperature and pressure under normal body conditions.

By taking a first - order Taylor series expansion to linearization the mass balance nonlinear differential nonlinear equation in the work by Yu is given as

$$
\begin{gathered}
\dot{P}_{A} V_{A}=\dot{P}_{A} V_{o, A}+\Delta \dot{P}_{A} V_{A} . \\
P_{I} \dot{V}_{A}=P_{o, I} \dot{V}_{A}+P_{I} \Delta \dot{V}_{A} . \\
P_{A} \dot{V}_{A}=P_{o, A} \dot{V}_{A}+P_{A} \Delta \dot{V}_{A} \\
C_{v}=C_{o, v}+\beta_{v} \Delta P_{v} . \\
C_{c}=C_{o, c}+\beta_{c} \Delta P_{A} . \\
\dot{P}_{A} V_{o, A}+\Delta \dot{P}_{A} V_{A}=P_{o, I} \dot{V}_{A}+P_{I} \Delta \dot{V}_{A}-P_{o, A} \dot{V}_{A}-P_{A} \Delta \dot{V}_{A}+8.63 Q_{p}\left(\left(C_{o, v}+\right.\right. \\
\left.\left.\beta_{v} \Delta P_{v}\right)-\left(C_{o, c}+\beta_{c} \Delta P_{A}\right)\right) .
\end{gathered}
$$

With initial value is zero we get

$$
\Delta \dot{P}_{A} V_{A}=P_{I} \Delta \dot{V}_{A}-P_{I} \Delta \dot{V}_{A}+8.63 Q_{p}\left(\beta_{v} \Delta P_{v}-\beta_{c} \Delta P_{A}\right)
$$

Where $P_{v}$ is the partial pressure of oxygen in the venous blood and is assumed equal to the partial pressure in the tissue compartment, $P_{T}$. By substituting Eq (2.1) and (2.2) in (2.7) and get 


$$
\begin{gathered}
\Delta \dot{P}_{A} V_{A}=\left(1-x_{d}\right) \dot{V}_{I} \Delta P_{I}-\Delta P_{A}\left(1-x_{d}\right)+8.63\left(1-y_{s}\right) Q\left(\beta_{v} \Delta P_{v}-\right. \\
\left.\beta_{c} \Delta P_{A}\right) \\
\Delta \dot{P}_{A} V_{A}=\left(1-x_{d}\right) \dot{V}_{I}\left(\Delta P_{I}-\Delta P_{A}\right)+8.63\left(1-y_{s}\right) Q\left(\beta_{v} \Delta P_{T}-\beta_{c} \Delta P_{A}\right) .
\end{gathered}
$$

It is shown that the system can be modeled in as an open - loop system without the feedback of the partial pressure of oxygen in the tissue as was done by $\mathrm{Yu}$ [12]. This assumption eliminates the $\Delta P_{T}$ term from Eq (2.9) and gets

$$
\Delta \dot{P}_{A} V_{A}=\left(1-x_{d}\right) \dot{V}_{I}\left(\Delta P_{I}-\Delta P_{A}\right)-8.63\left(1-y_{s}\right) Q \beta_{c} \Delta P_{A}
$$

At the steady state space gain that leads that term in the left side will be zero and after that we get

$$
\begin{gathered}
\left(1-x_{d}\right) \dot{V}_{I}\left(\Delta P_{I}-\Delta P_{A}\right)=8.63\left(1-y_{s}\right) Q \beta_{c} \Delta P_{A} . \\
(2.11) \\
\left(1-x_{d}\right) \dot{V}_{I} \Delta P_{I}-\left(1-x_{d}\right) \dot{V}_{I} \Delta P_{A}=8.63\left(1-y_{s}\right) Q \beta_{c} \Delta P_{A} . \\
(2.12) \\
\left(1-x_{d}\right) \dot{V}_{I} \Delta P_{I}=\left(1-x_{d}\right) \dot{V}_{I} \Delta P_{A}+8.63\left(1-y_{S}\right) Q \beta_{c} \Delta P_{A} .
\end{gathered}
$$

By finding the ratio between $\Delta P_{A}$ to $\Delta P_{I}$ we get 


$$
\frac{\Delta P_{A}}{\Delta P_{I}}=\frac{\left(1-x_{d}\right) \dot{V}_{I}}{8.63\left(1-y_{S}\right) Q \beta_{c}+\left(1-x_{d}\right) \dot{V}_{I}} .
$$

The variation in $P_{a}$ per unit change in alveolar oxygen over a variation in the oxygen content due to the intrapulmonary shunt is

$$
\frac{\Delta P_{a}}{\Delta P_{A}}=\frac{\left(1-y_{s}\right) \beta_{c}}{\beta_{a}}
$$

The fractional composition of a gas is related to its partial pressure as

$$
\frac{\Delta P_{I}}{\Delta F_{I}}=P_{B}-P_{H 2 o}
$$

where $P_{H 2 o}$ is water vapor pressure and $P_{B}$ is the barometric pressure. We can get the steady state gain is

$$
G p=\frac{\Delta P_{A}}{\Delta P_{I}} \frac{\Delta P_{a}}{\Delta P_{A}} \frac{\Delta P_{I}}{\Delta F_{I}}=\frac{\left(1-x_{d}\right) \dot{V}_{I}}{8.63\left(1-y_{S}\right) Q \beta_{c}+\left(1-x_{d}\right) \dot{V}_{I}} \frac{\left(1-y_{s}\right) \beta_{c}}{\beta_{a}}\left(P_{B}-P_{H 2 o}\right) .
$$

Where the parameter $\beta_{a}$ is the equivalent to the slope of the tangent line of the oxygen dissociation curve at the current partial pressure of oxygen in artery.

from $\mathrm{Eq}(10)$ can we get the homogenous equation as

$$
\frac{V_{A}}{\left[\left(1-x_{d}\right) \dot{V}_{I}+8.63\left(1-y_{s}\right) Q \beta_{c}\right]} \Delta \dot{P}_{A}+\Delta P_{A}=0
$$

Where the parameter $\beta_{c}$ is the apparent solubility of oxygen in whole blood in the alveolar. From Eq (10) a time constant for the lung can be

$$
\tau=\frac{V_{A}}{8.63\left(1-y_{s}\right) \dot{Q}_{P} \beta_{c}+\left(1-x_{d}\right) \dot{V}_{I}}=\frac{V_{A}}{\dot{V}_{A}+8.63 \dot{Q}_{P} \beta_{c}} .
$$


We can find the time constant at nominal condition when using nominal system parameters.

A linearized model of the respiratory system of neonatal infants is derived as

$$
\tau \Delta P_{a} s+\Delta P_{a}=G_{p} \Delta \mathrm{Fio}_{2}
$$

Where $G_{p}$ is the steady state system gain, $\Delta P_{a}$ is the linearized partial pressure of oxygen in the lung. The parameters for the linear system are computed at nominal conditions. To modify the system such that the output is $O_{2}$, the oxygen dissociation curve that was derived by Severinghaus is evaluated at the partial pressure of oxygen in the artery, $P_{a}[11]$. The oxygen dissociation curve is defined by

$$
S p O_{2}=\frac{1}{23400\left[P_{a}^{3}+150 P_{a}\right]^{-1}+1}(100 \%) .
$$

The curve of the oxygen dissociation can be seen in Figure 2. The derivative of equation (2.21) with respect to $P_{a}$ is computed at nominal conditions curve gain, $G_{c}$, is used to convert $P_{a}$ to $\mathrm{SpO}_{2}$ by

$$
\begin{gathered}
\Delta \mathrm{SpO}_{2}=G_{c} \Delta \mathrm{PaO} \cdot 2 \\
\Delta S \dot{p} O_{2}=G_{c} \Delta \dot{P} a O_{2} .
\end{gathered}
$$

From $\mathrm{Eq}(2.22)$ we can get $\triangle \mathrm{PaO}$ as

$$
\Delta \mathrm{PaO}_{2}=\frac{\Delta \mathrm{SpO}_{2}}{G_{c}} .
$$

By substitution equation from (2.23) in (2.20) and get 


$$
\begin{gathered}
\tau \frac{\Delta S p O_{2}}{G_{c}} s+\frac{\Delta S p O_{2}}{G_{c}}=G_{p} \Delta F i o_{2} . \\
\tau \Delta S p O_{2} s+\Delta S p O_{2}=G_{p} G_{c} \Delta F i o_{2} . \\
\tau \Delta S p O_{2} s+\Delta S p O_{2}=G_{p c} \Delta F i o_{2} . \\
G_{p c}=G_{p} G_{c} .
\end{gathered}
$$

We can solve $\beta$ parameters from the derivation of the oxygen dissociation curve and is evaluated for the range of partial pressure of oxygen and is given by the equation

$$
\frac{d s p O_{2}}{d P_{a}}=\frac{23400\left(3 P_{a}^{2}+150\right)}{\left(P_{a}^{3}+150 P_{a}\right)^{2}\left(\frac{23400}{P_{a}{ }^{3}+150 P a}+1\right)^{2}} .
$$

In Figure 3, we can show the graph of the derivative of the oxygen dissociation curve. In the alveolar capillary that the partial pressure of oxygen is not known from this model and is solved for based on the output $P_{a}$. The alveolar capillary partial pressure is solved for using

$$
P_{A} O_{2}=P_{a} O_{2}+K_{a}
$$

where $K_{a}$ is the alveolar - arterial oxygen difference [9]. The alveolar - arterial oxygen difference is assumed to be $1.5 \%$ which is the nominal difference between the two saturations. By using Eq (2.27) and the oxygen dissociation curve, the alveolar capillary partial pressure can be determined. 


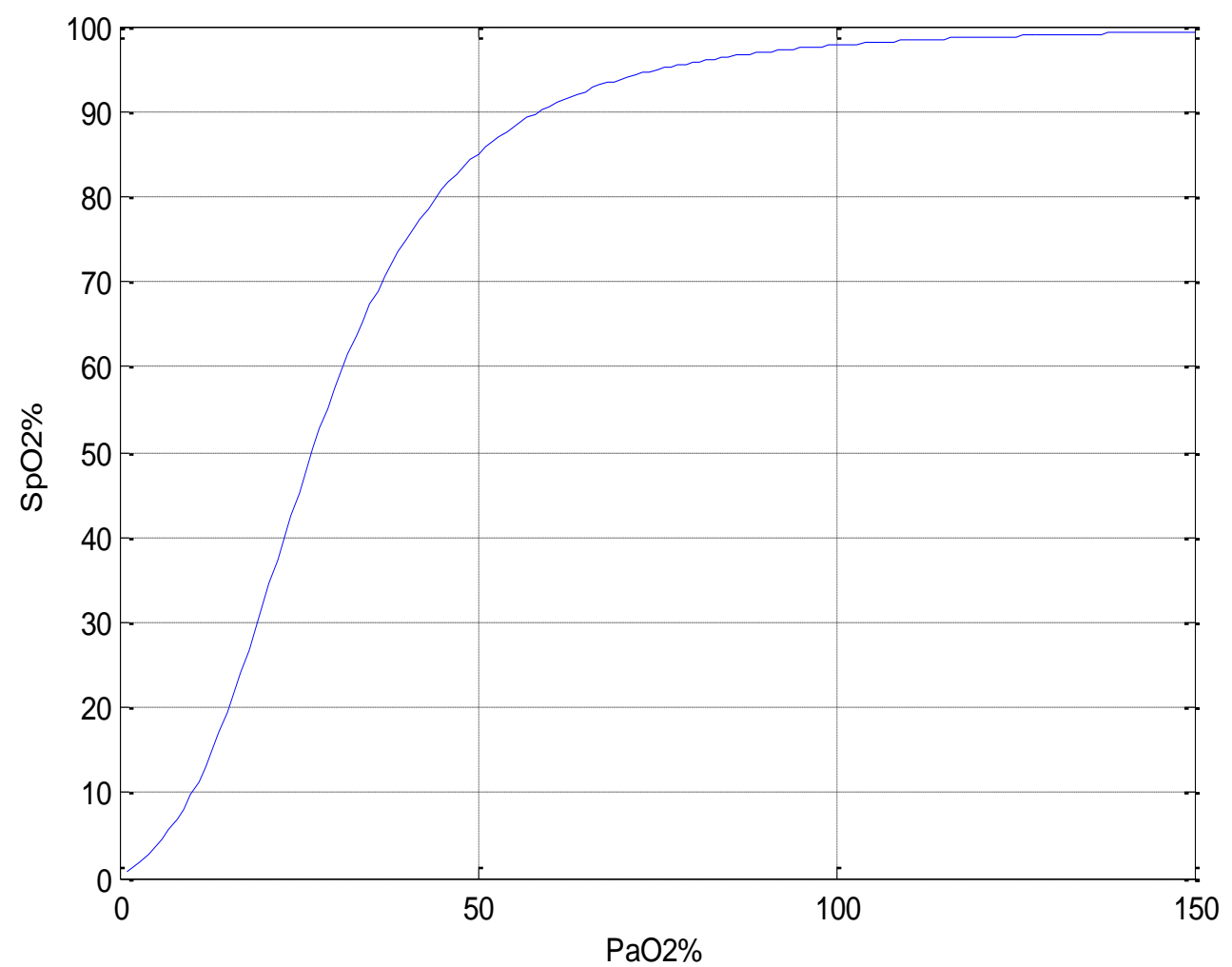

Figure 3. The Graph of the oxygen dissociation curve.

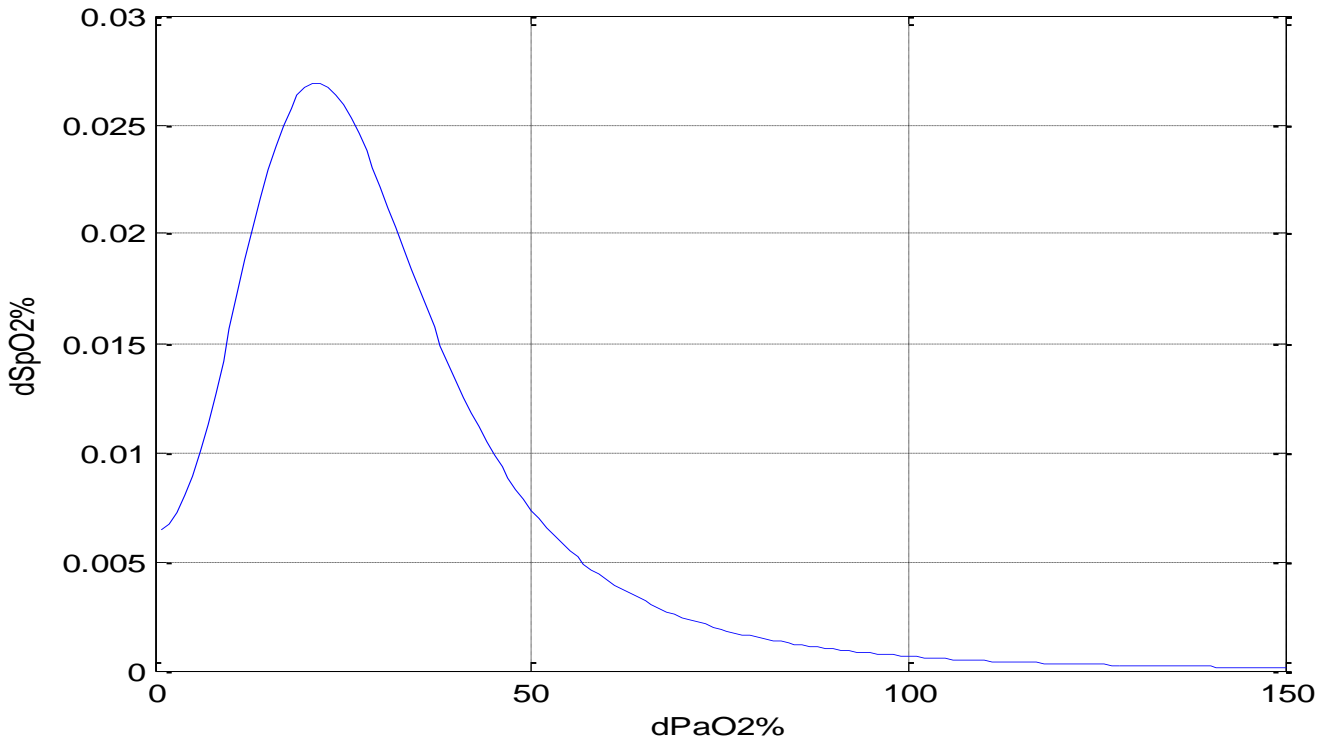

Figure 4. Graph showing the derivative of the oxygen dissociation curve. 
The linear model is expanding to include time varying parameters. Instead of keeping $\beta_{c}$ and $\beta_{a}$ constant, these parameters are allowed to vary based on the output $P_{a}$. By computing the derivative of the oxygen dissociation curve we can solve the $\beta$ parameters at the current partial pressure of oxygen. By combining Eqs (2.17), (2.19), and (2.26), we can form the nonlinear system model and by including nonlinear terms and is given by

$$
\frac{V_{A}}{\dot{V}_{A}+8.63 \dot{Q}_{P}} \Delta S p O_{2} S+\Delta S p O_{2}=\frac{\left(1-x_{d}\right) \dot{V}_{I}\left(1-y_{s}\right) \beta_{c}\left(P_{B}-P_{H 2 o}\right)}{\left(8.63\left(1-y_{s}\right) Q \beta_{a}+\left(1-x_{d}\right) \dot{V}_{I}\right)} \Delta F i O_{2}
$$

From Eq (2.30) we can divide to three parts, the first part is the time constant, $\tau$, gain, $G_{p}$, and and can see it in Figure ( 5) -(7)

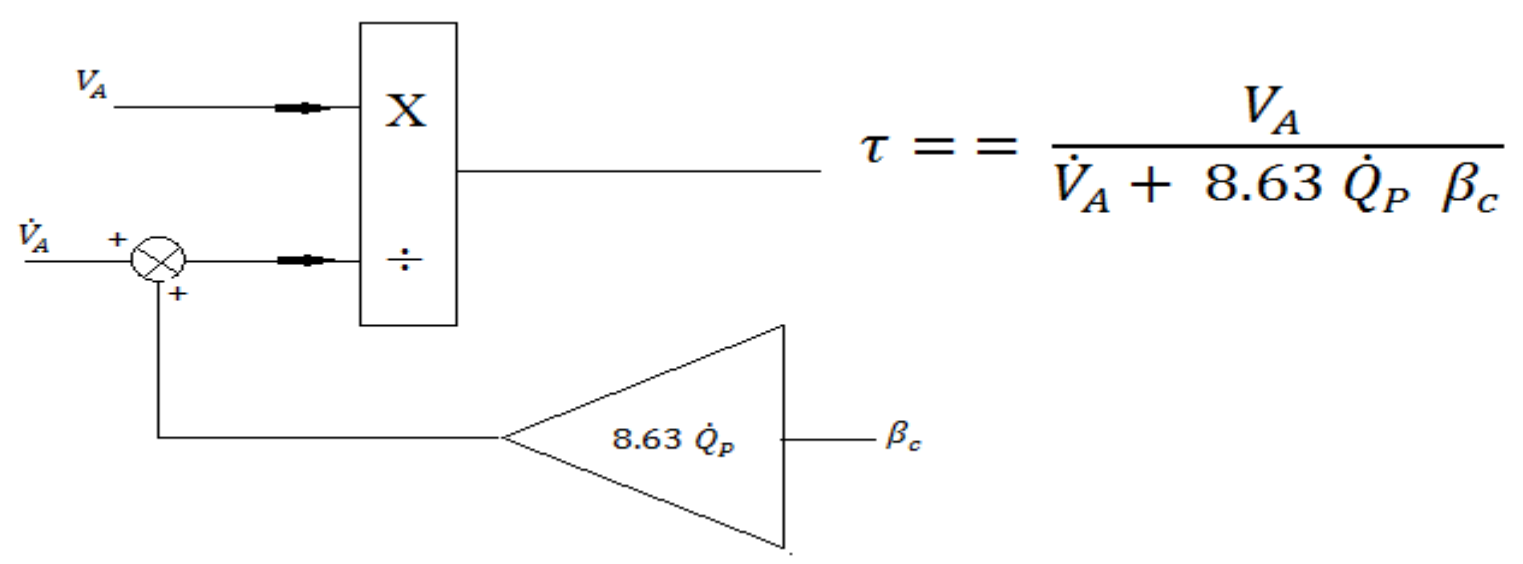

Figure 5. The block diagram of time constant $\tau$. 


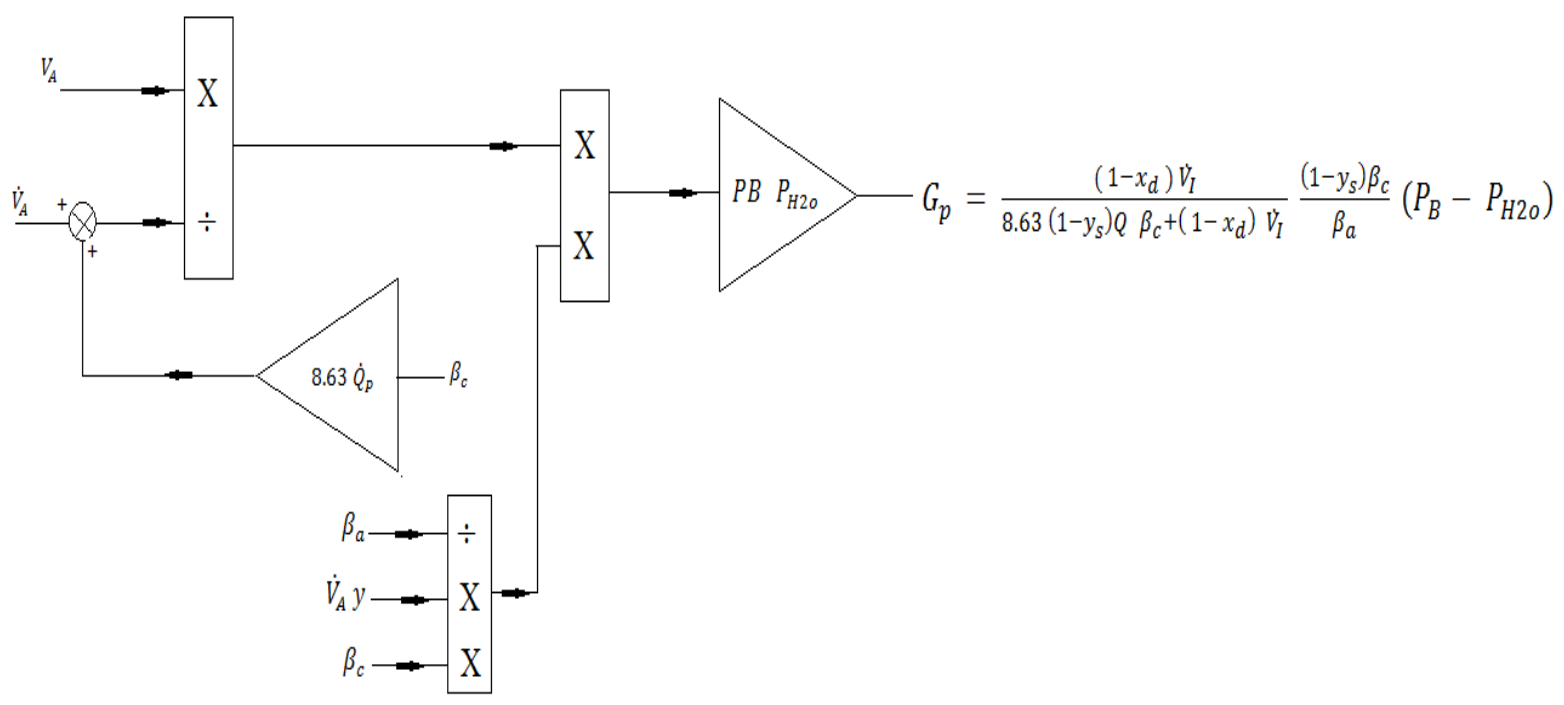

Figure 6. The block diagram of gain, $G_{p}$.

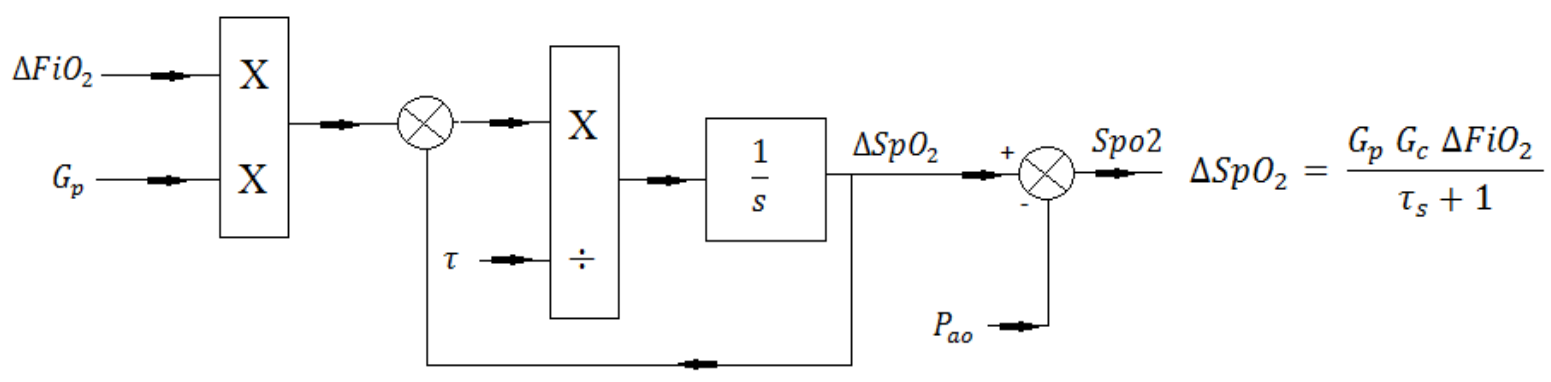

Figure 7. The block diagram of $\Delta \mathrm{SpO}_{2}$.

For different step inputs, we can compare the nonlinear and linear model. The values of nominal values of $\beta_{c}$ and $\beta_{a}$ are different in linear and nonlinear according to $\mathrm{Yu}$ and Batzal paper. The nominal value for the parameter $\beta_{c}$ in the linear model is $0.0105 \mathrm{vol} \% / \mathrm{mm} \mathrm{Hg}$ and the nominal value for the parameter $\beta_{a}$ in linear model is $0.0166 \mathrm{vol} \% / \mathrm{mm} \mathrm{Hg}$. These $\beta$ parameters vary for the nonlinear model. Now, we can compare the linear and nonlinear models when we supply $0.1 \% \mathrm{FiO}_{2}$ step input is chosen for the first simulation, and an $8 \% \mathrm{FiO}_{2}$ is 
chosen for the second. The nominal conditions for the system parameters are taken from $\mathrm{Yu}$ and Batzel and can be seen in Table 1 [16].

Table 1. The nominal parameters that we took from $\mathrm{Yu}$ and Batzal papers

\begin{tabular}{|c|c|}
\hline Parameter & Nominal value \\
\hline$V_{A}$ & $0.491 \mathrm{~L}$ \\
\hline$\dot{V}_{A}$ & $4-6 \mathrm{~L} / \mathrm{min}$ \\
\hline $\mathrm{Q}$ & $5 \mathrm{~L} / \mathrm{min}$ \\
\hline$x_{d}$ & $5 \%$ \\
\hline$y_{S}$ & $5 \%$ \\
\hline$P_{B}$ & 760 torr \\
\hline$P_{H 2 o}$ & 47 torr \\
\hline
\end{tabular}

Now, the parameters in the Table above put to Eq (2.30) to get the output $\mathrm{SpO}_{2}$ from the linear system model when supplying a $\mathrm{FiO}_{2}$ step input as in Figure 8 . 


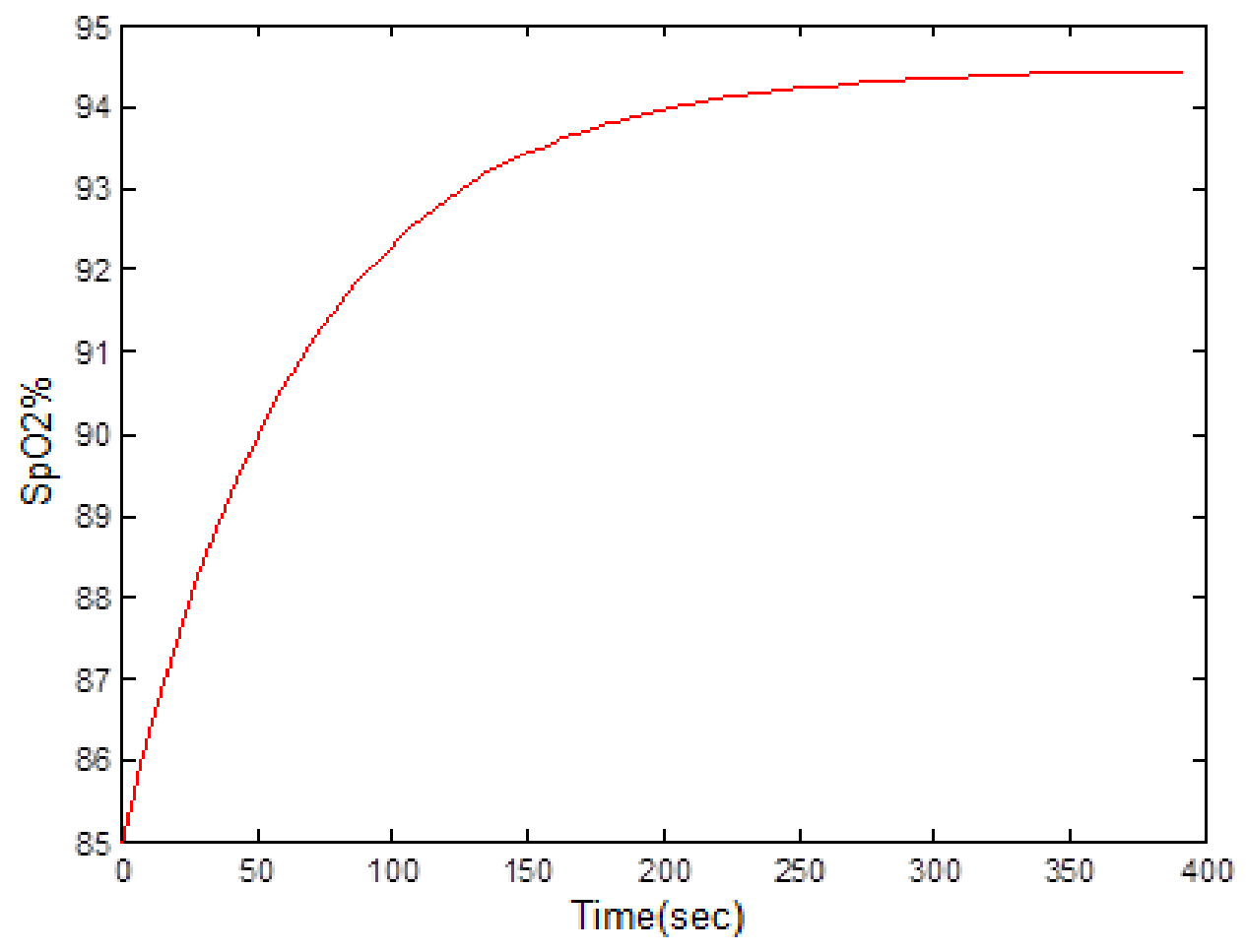

Figure 8. Output $\mathrm{SpO} 2$ from the linear system models when supplying FiO2 step input. 


\section{Chapter 3: Controller of the System}

This chapter describes three controller methods; Proportional - integral (PI), Proportional - Integral - Derivative (PID) and Model Predictive Control (MPC). Theses controllers need algorithms to define how the control action will affect the behavior of the output response and we derive each controller to be input $\mathrm{FiO}_{2}$ to the plant to get output $\mathrm{SpO}_{2}$.

\subsection{Proportional Integral (PI) Controller.}

A fixed proportional - integral (PI) controller was connected in feed forward to control arterial oxygen saturation $\mathrm{SpO}_{2}$ by adjusting inspired oxygen fraction, $\mathrm{FiO}_{2}$. The performance of the feedback system was found to be sensitive to the open-loop plant gain. To get an acceptable transient behavior, the controller was tuned using trial and error selection of $K_{p}$ and $K_{d}$ in the closed - loop system as in Fig 9. 


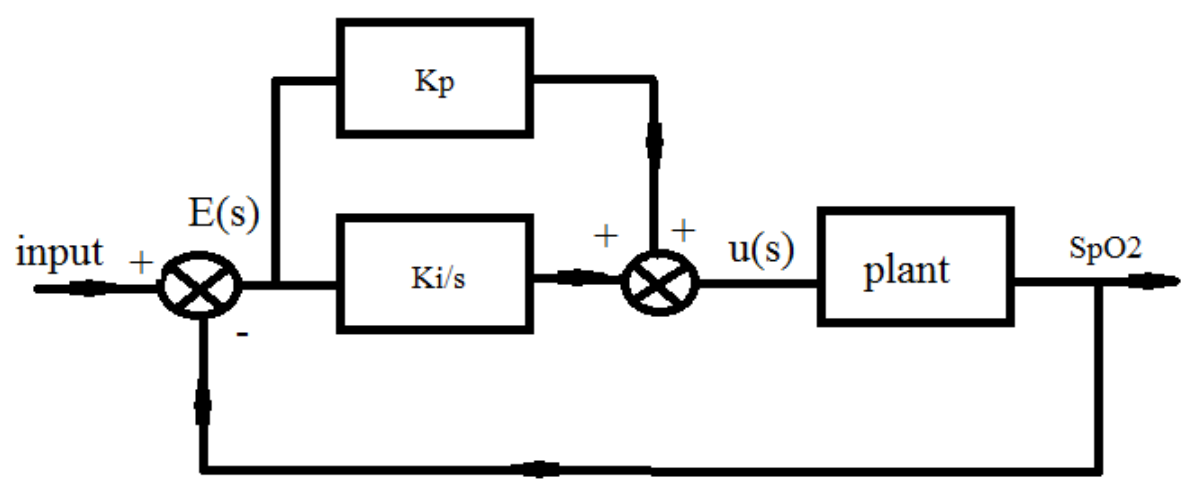

Figure 9. The Block diagram of PI Controller.

The differential equation of the continuous PI controller is

$$
u(s)=K_{p} e(s)+K_{i} / s e(s)
$$

The Bilinear method to convert the continuous Laplace transform to discrete Ztransform equation is applied by substituting

$$
s=\frac{2}{T} \frac{z-1}{z+1}
$$

After finding the Z-transform, the discrete equation of the digital PI controller is found to be

$$
u(n)=2 u(n-1)+K_{p} e(n)-K_{p} e(n-1)+0.5 K_{i} T e(n)+0.5 K_{i} T e(n-1)
$$


where $u(n)$, is an output of the controller has an upward physical limit of $100 \% \mathrm{O}_{2}$ and a lower bound of $21 \% \mathrm{O}_{2}, T$ is the discrete sampling time $T$ is 1 sec. By tuning $K_{p}$ and $K_{i}$ we get good performance and minimum peak over shoot and zero steady state error for $\mathrm{SpO} 2$ output. Simulation results using the PI control are in Fig (10) - Fig (19).
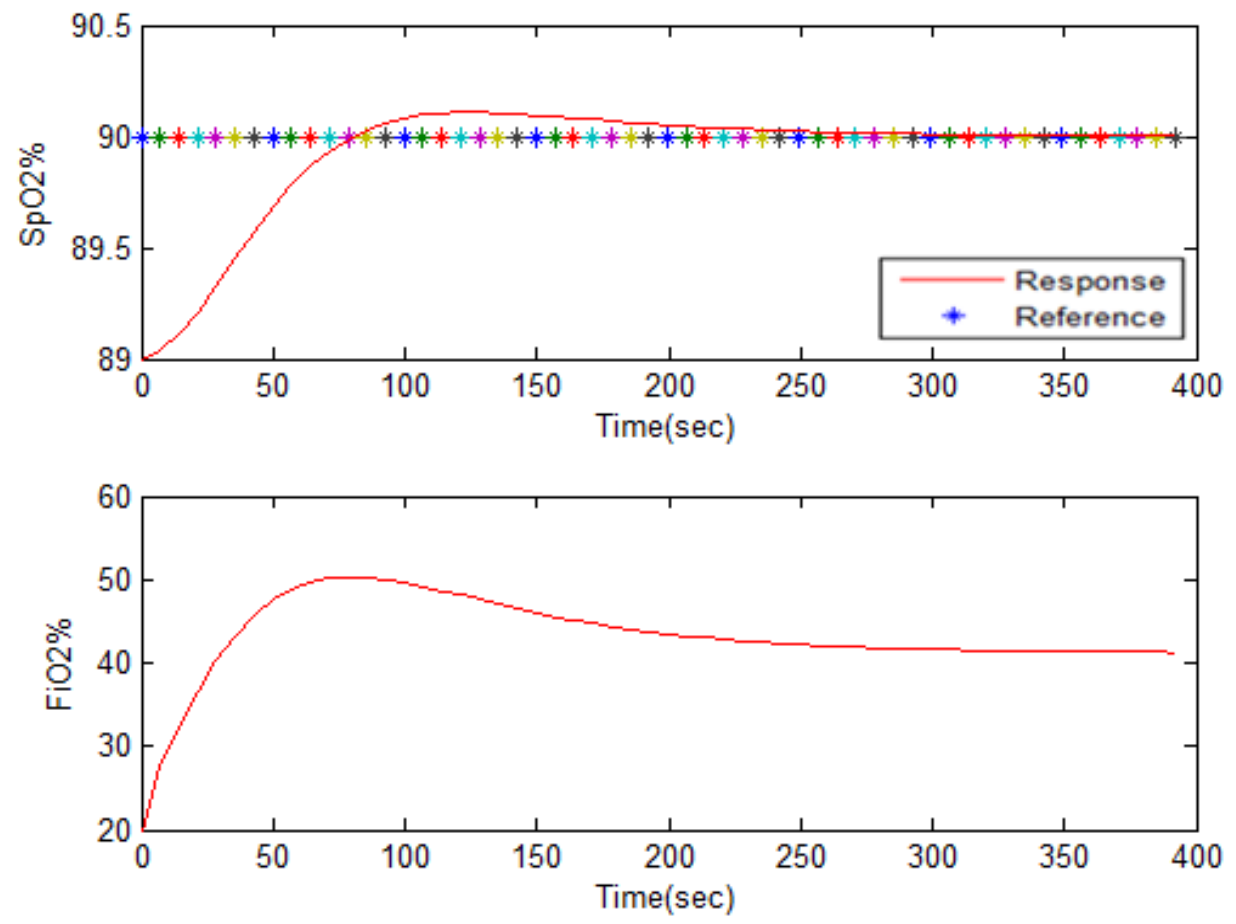

Figure 10. Simulated closed - loop $\mathrm{SpO2}$ with $\mathrm{Kp}=0.5$ and $\mathrm{Ki}=5$. 

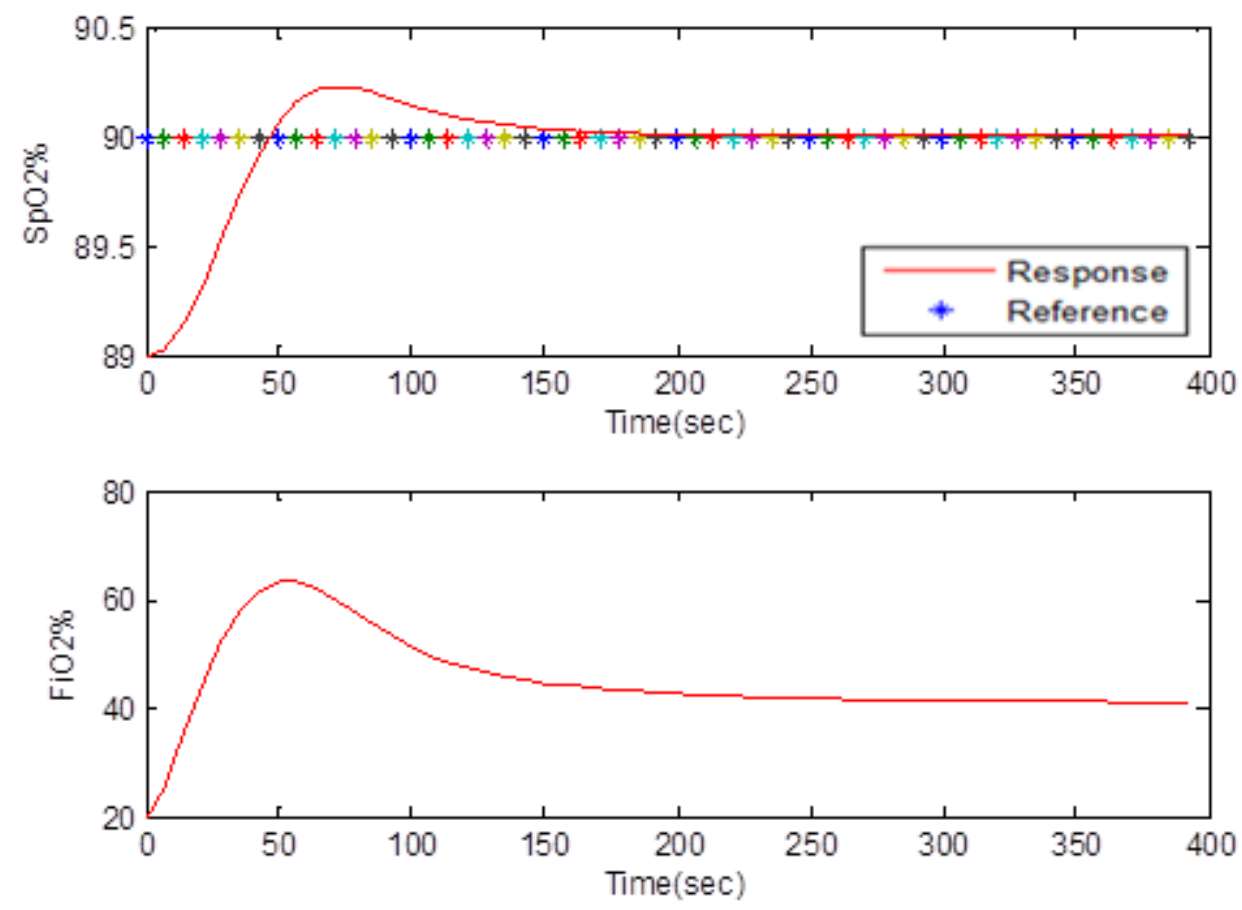

Figure 11. Simulated closed $-\operatorname{loop} \mathrm{SpO2}$ with $\mathrm{Kp}=0.005$ and $\mathrm{Ki}=10$.
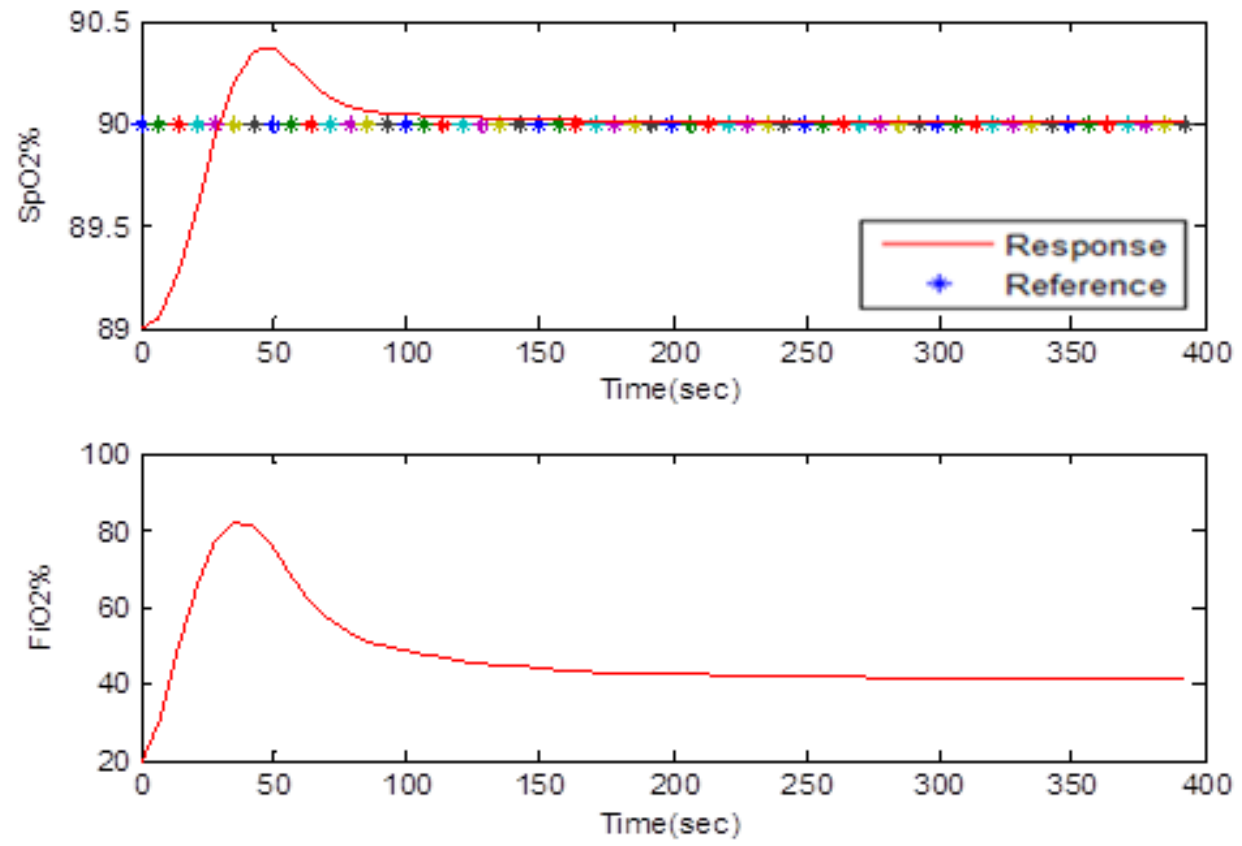

Figure 12. Simulated closed $-\operatorname{loop} \mathrm{SpO2}$ with $\mathrm{Kp}=0.0005$ and $\mathrm{Ki}=20$. 

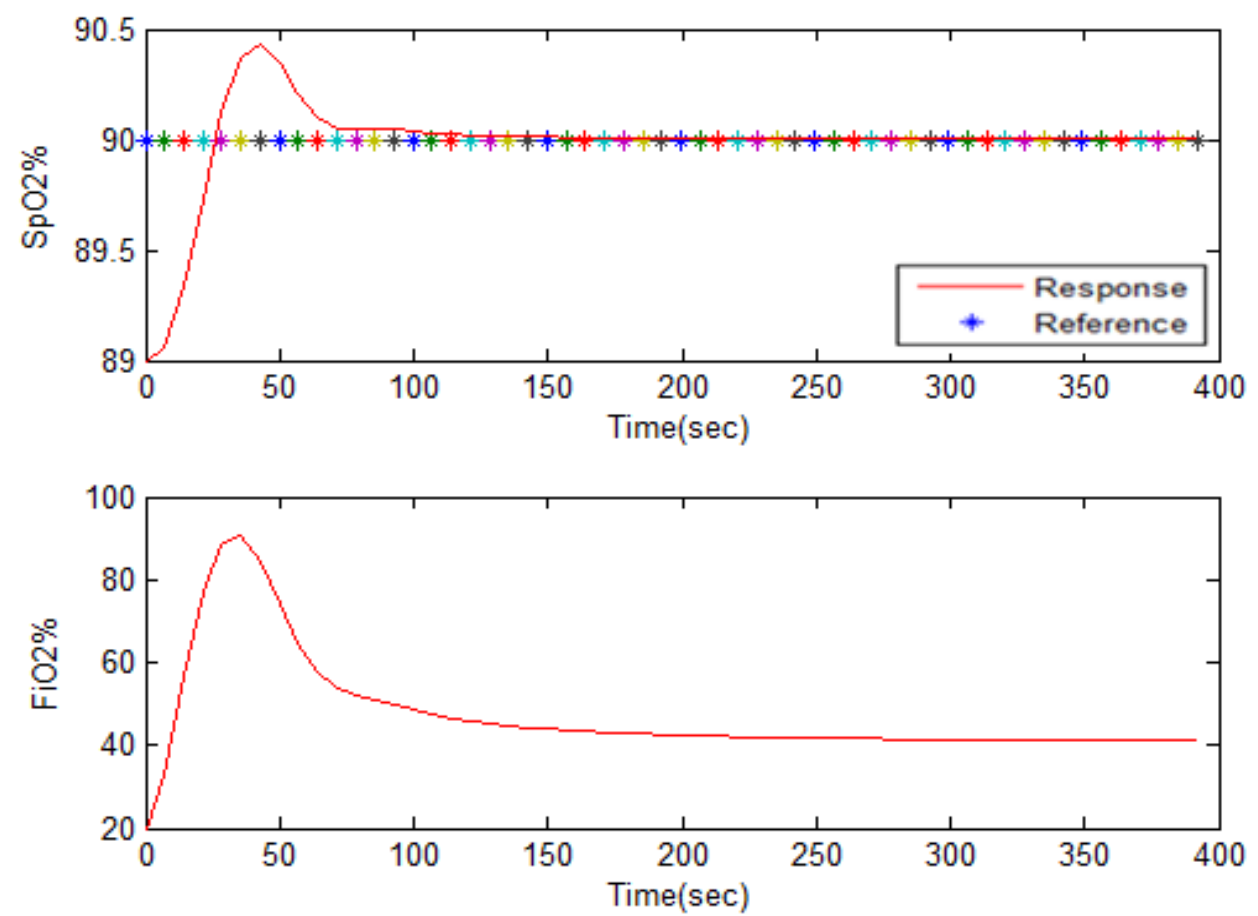

Figure 13. Simulated closed - loop SpO2 with $\mathrm{Kp}=0.0005$ and $\mathrm{Ki}=25$.
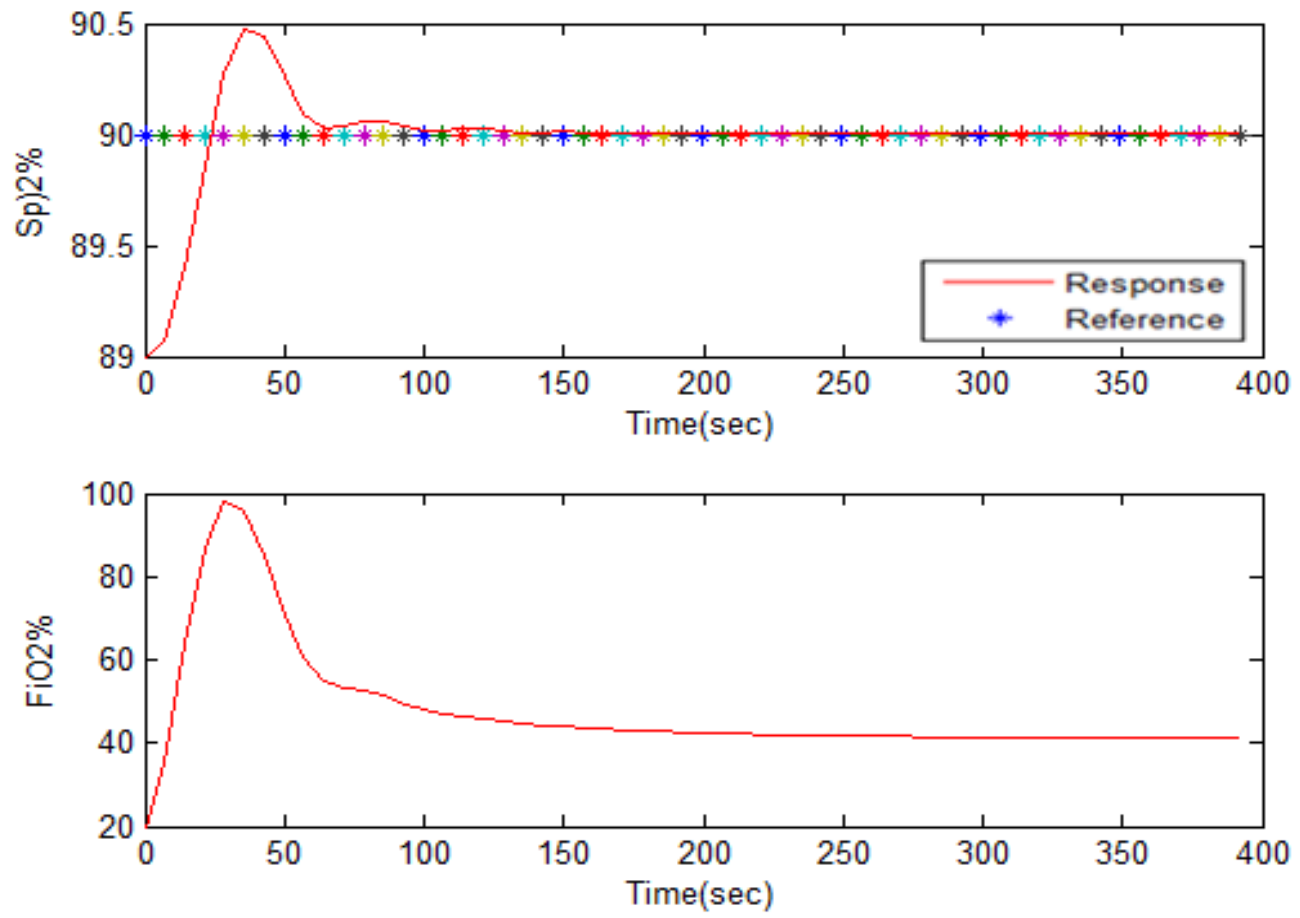

Figure 14. Simulated closed - loop $\mathrm{SpO} 2$ with $\mathrm{Kp}=\mathbf{0 . 0 0 0 5}$ and $\mathrm{Ki}=30$. 

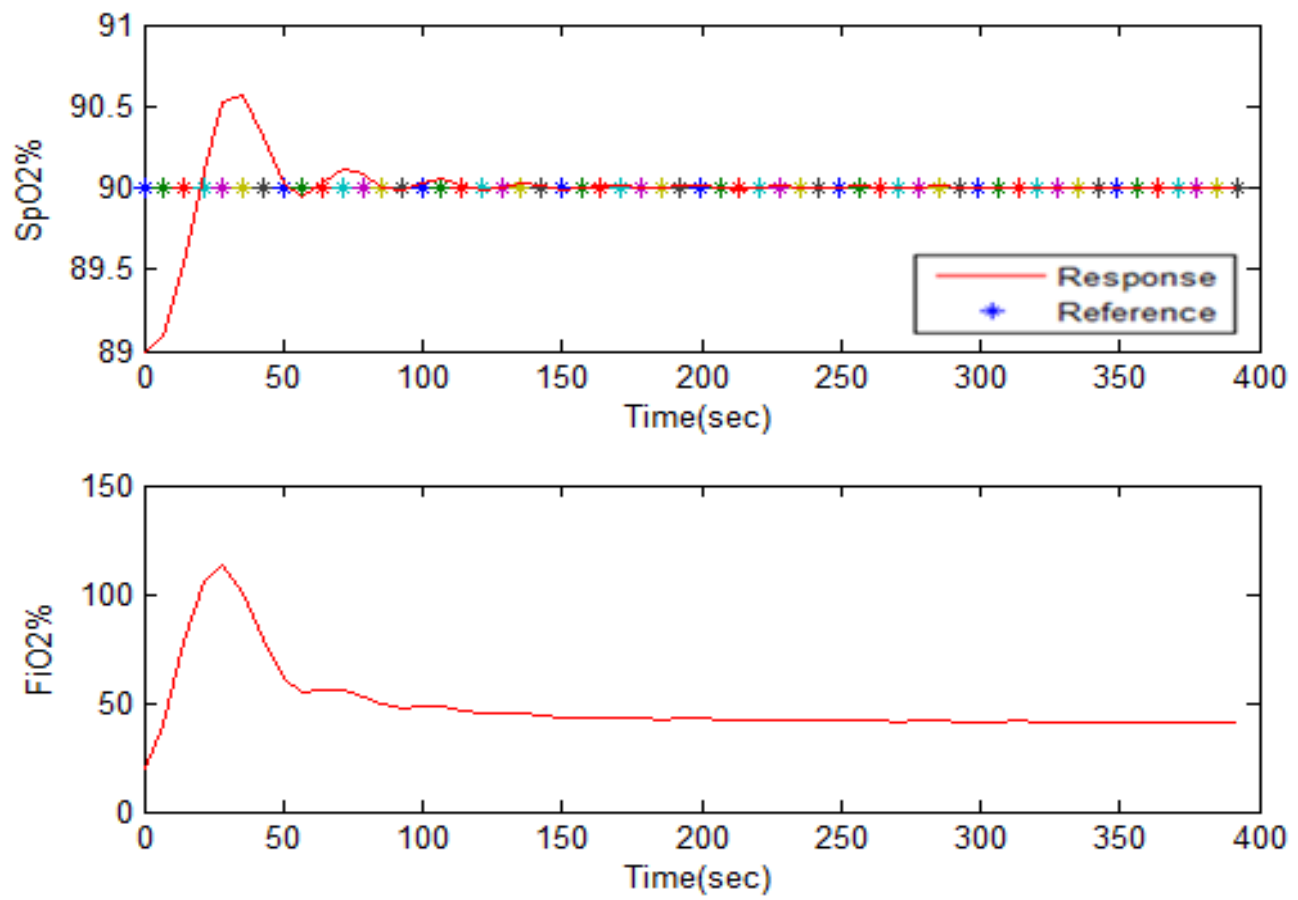

Figure 15. Simulated closed $-\operatorname{loop} \mathrm{SpO2}$ with $\mathrm{Kp}=0.0005$ and $\mathrm{Ki}=40$.
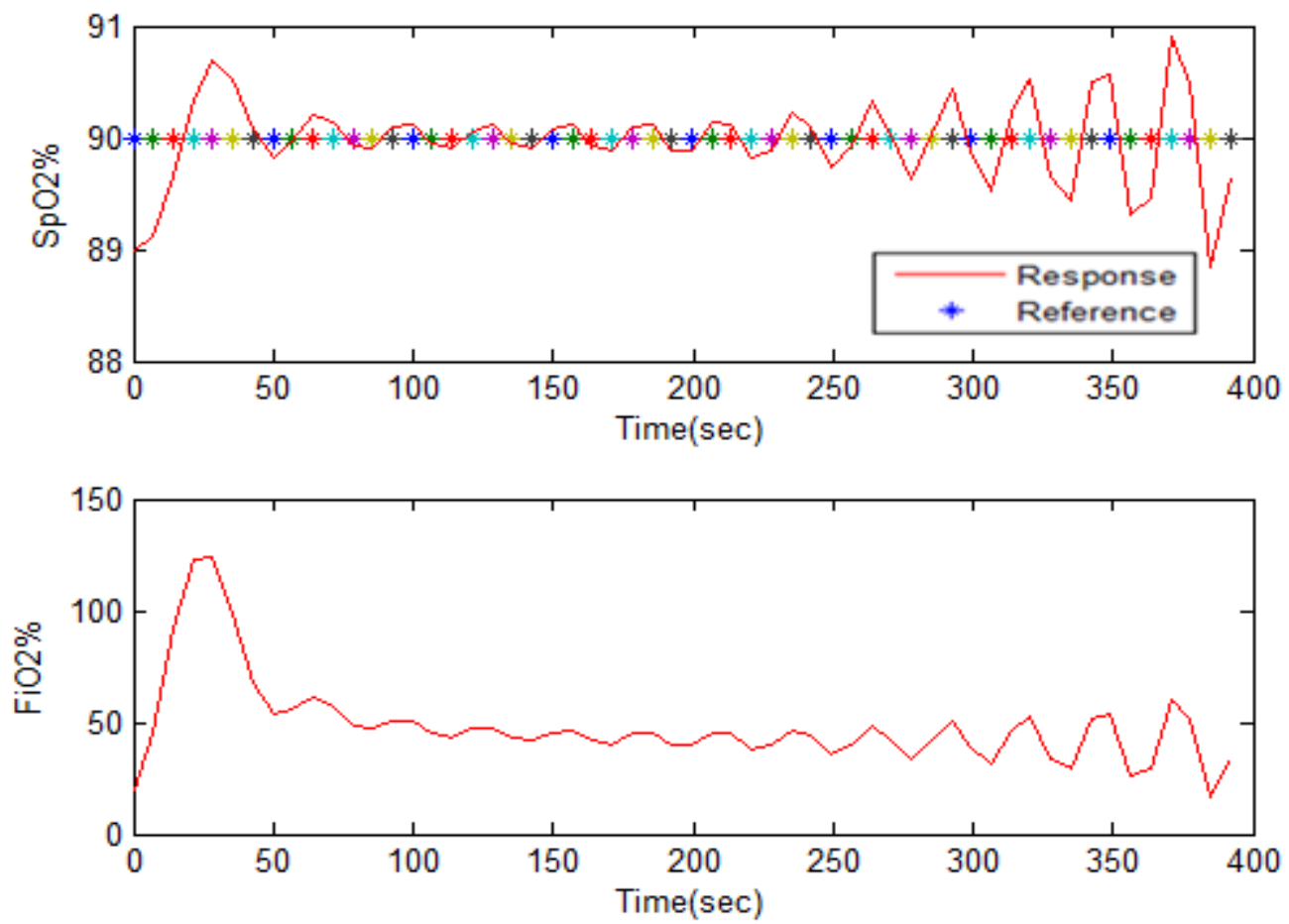

Figure 16. Simulated closed - loop $\mathrm{SpO2}$ with $\mathrm{Kp}=\mathbf{0 . 0 0 0 5}$ and $\mathrm{Ki}=\mathbf{5 0}$. 

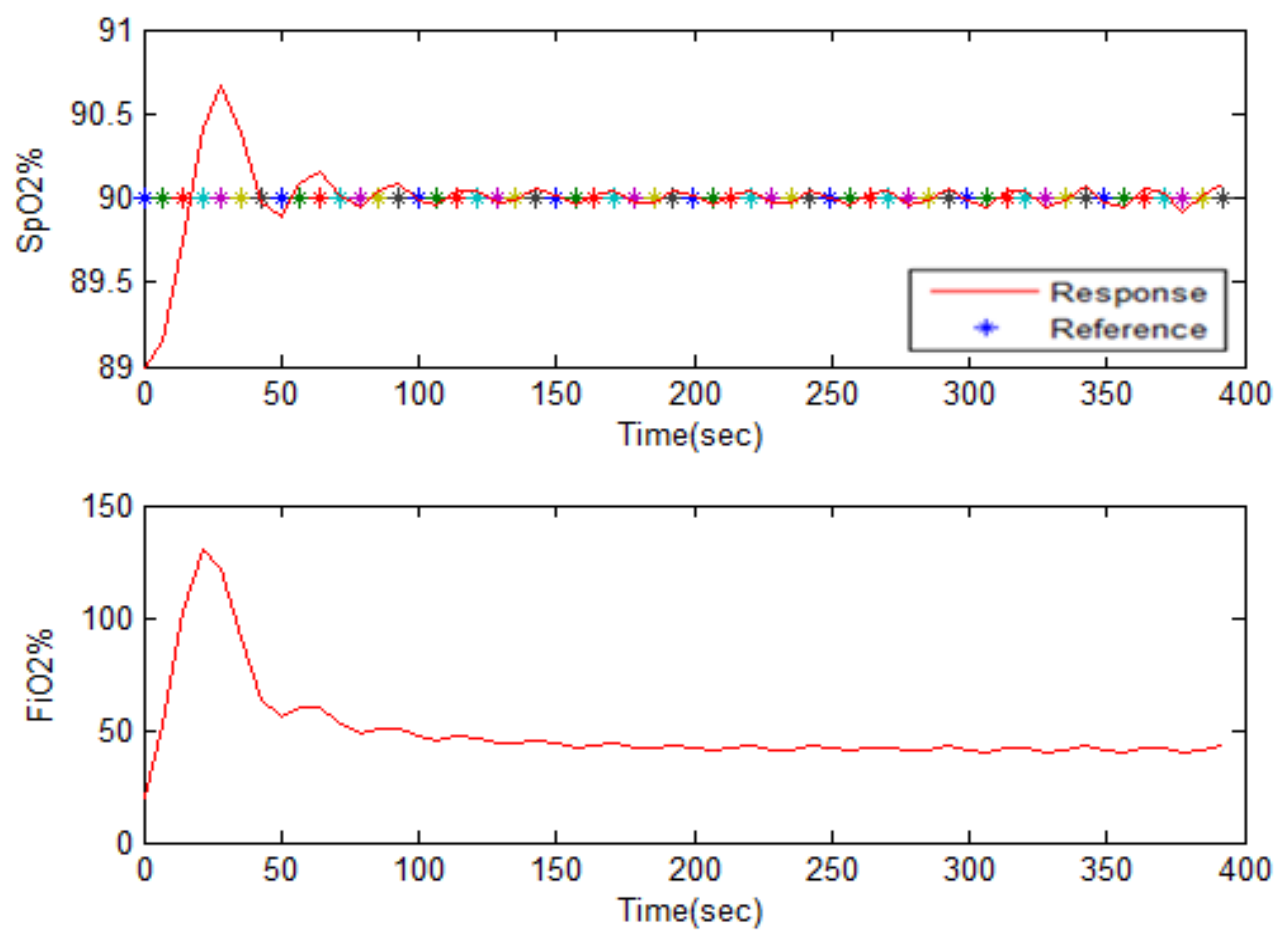

Figure 17. Simulated closed $-\operatorname{loop} \mathrm{SpO2}$ with $\mathrm{Kp}=\mathbf{0 . 0 5}$ and $\mathrm{Ki}=\mathbf{5 5}$.
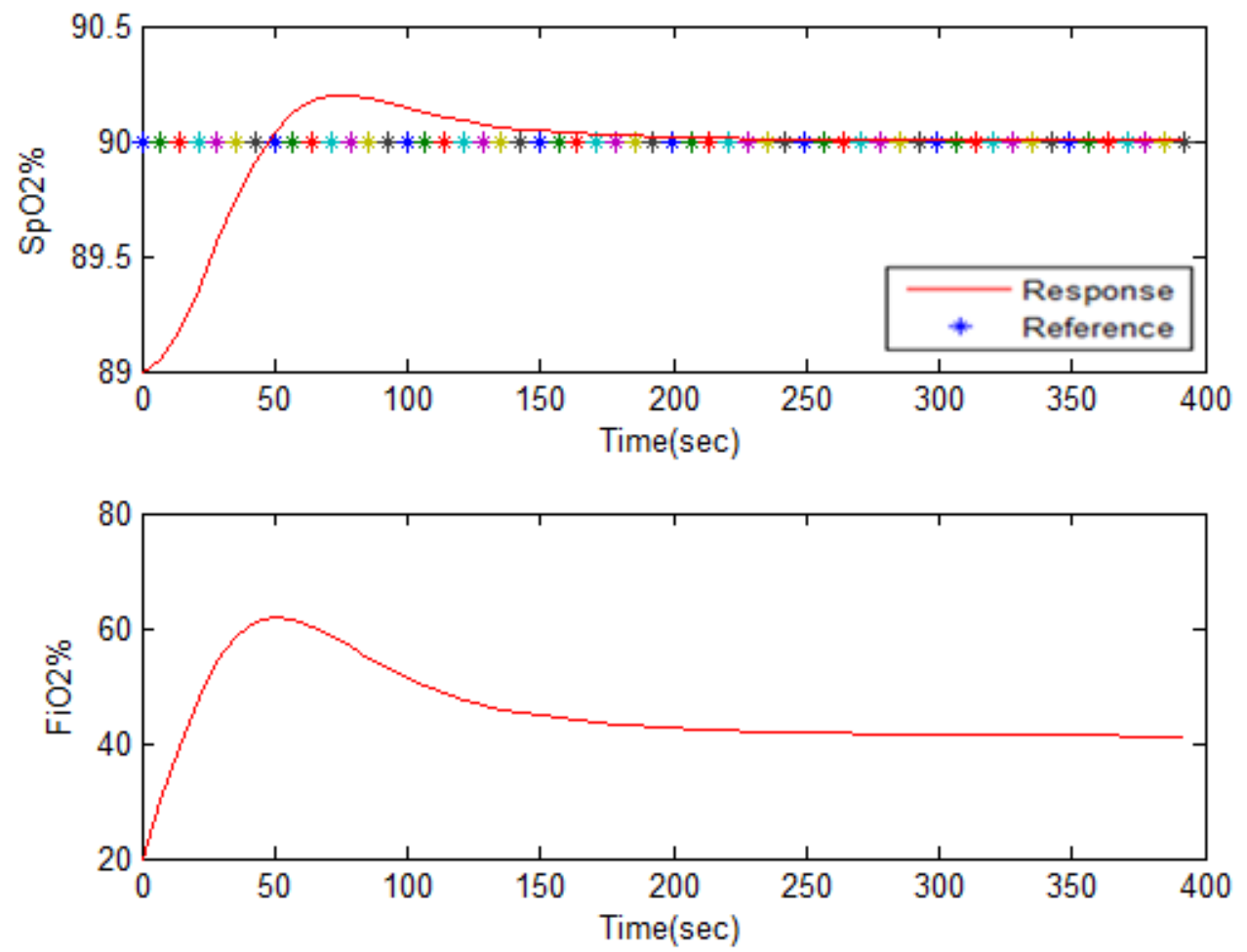

Figure 18. Simulated closed $-\operatorname{loop} \mathrm{SpO2}$ with $\mathrm{Kp}=\mathbf{0 . 0 5}$ and $\mathrm{Ki}=10$. 

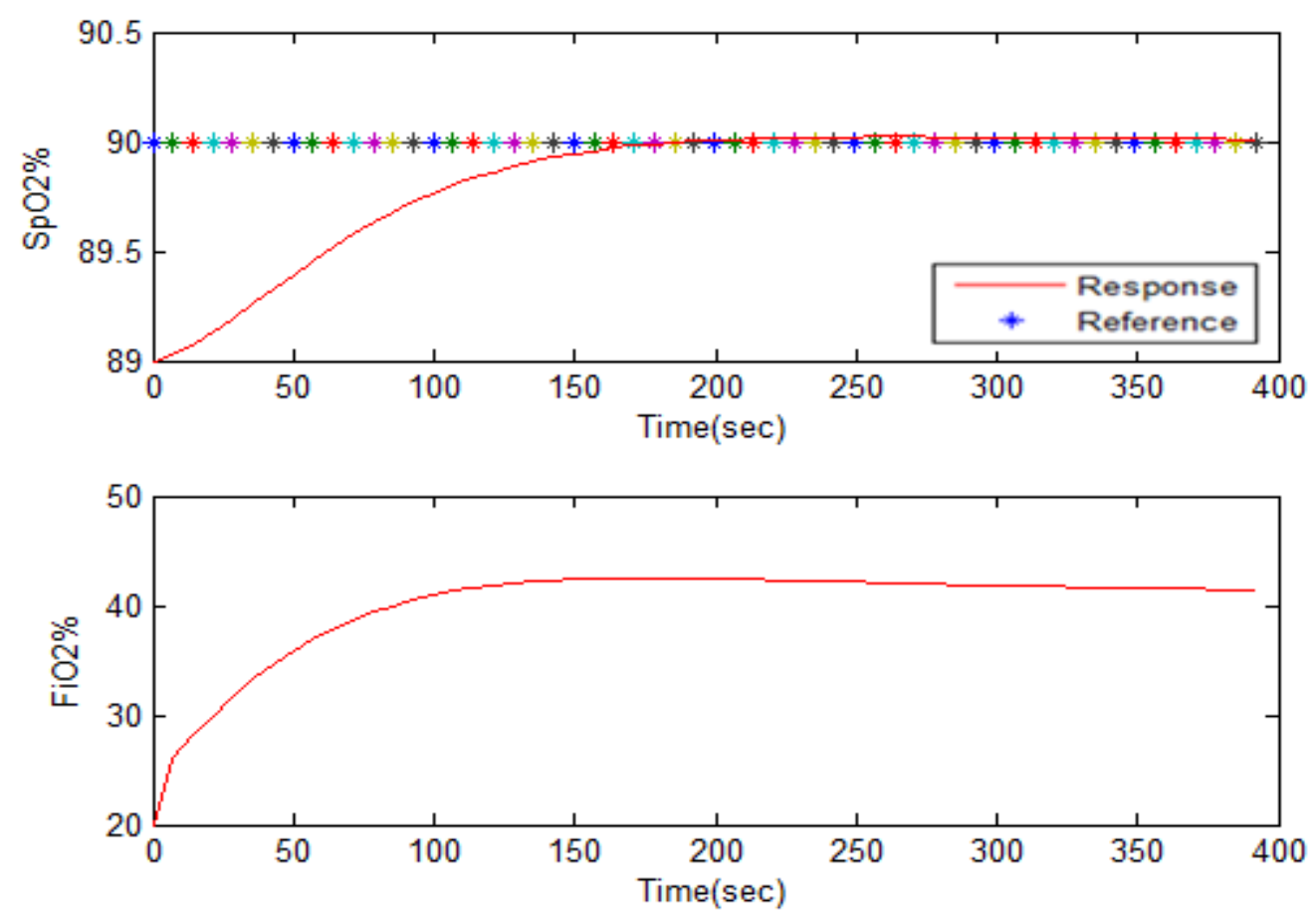

Figure 19. Simulated closed $-\operatorname{loop} \mathrm{SpO} 2$ with $\mathrm{Kp}=0.05$ and $\mathrm{Ki}=2.2$.

The control action in Eq (3.1) is simulated with tuning $K_{p}$ and $K_{i}$ by trial and error by Matlab codes and applied as input $\mathrm{FiO}_{2}$ to the plant to get $\mathrm{SpO}_{2}$ ranging from $85-93 \%$. In this test, the best response is in Fig 19 because there is no steady state error and minimum settling time and the value of $\mathrm{SpO}_{2}$ after 180 sec. 


\subsection{Proportional Integral Derivative (PID) Controller.}

The developed system uses a pulse oximeter for blood oxygen feedback signals and a computer program with a PID controller design as shown in Figure 20. It then sends a signal to a modified oxygen blender which delivers a specified FiO2 level to a newborn infant. The control was tuned by using the trial and error of $K_{p}, K_{i}$, and $K_{d}$ in the closed - loop system as in Fig 20.

The differential equation of continues PID controllers is

$$
u(s)=K_{p} e(s)+K_{i} / s e(s)+K_{s} s e(s)
$$

The Bilinear method to convert the continuous Laplace transform to discrete Ztransform equation is applied by substituting

$$
s=\frac{2}{T} \frac{z-1}{z+1}
$$

The discrete equation of the digital PID controller is

$$
\begin{aligned}
u(n)= & n(n-2)+\left(T k_{p}+0.5 T^{2} k_{i}+2 k_{d}\right) e(n)+\left(T k_{i}-4 k_{d}\right) e(n-1)+ \\
& \left(2 k_{d}+0.5 T k_{i}-T k_{p}\right) e(n-2)
\end{aligned}
$$


where $u(n)$ is control unit that put to the plant, $T$ is the sampling time to convert the continuous to discrete time and it was $0.1 \mathrm{sec}$. We got after simulation and by tuning values of $K_{p}, K_{i}$ and $K_{d}$ good performance and zero steady state error as in Fig 31 because the zero steady state error is zero and settling time is $170 \mathrm{sec}$.

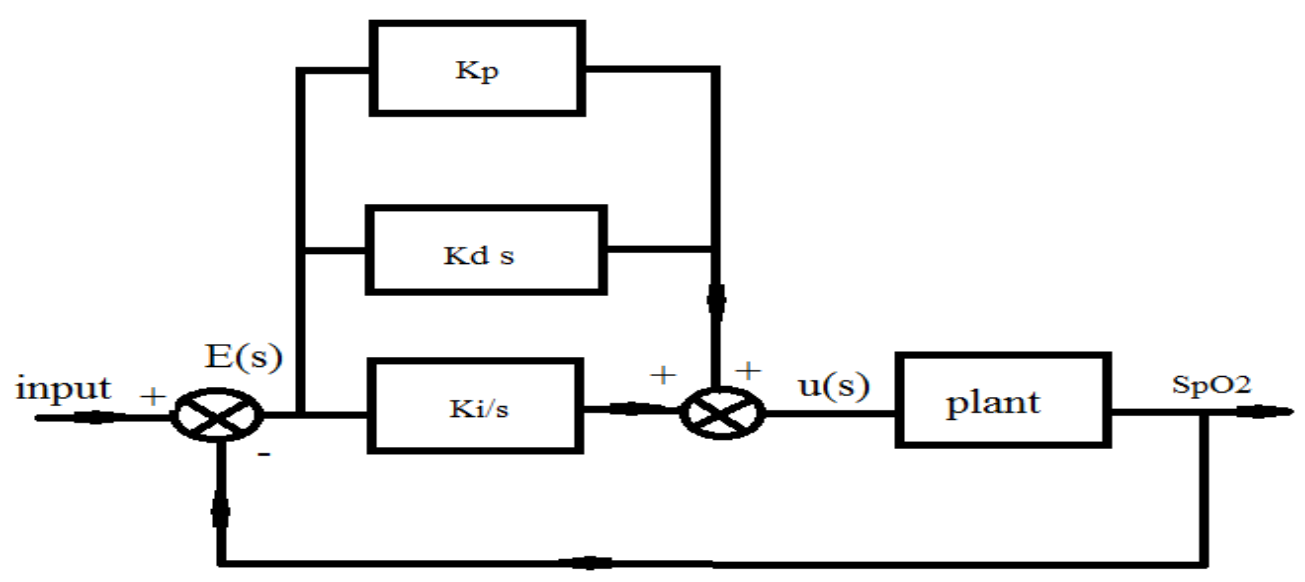

Figure 20. The block diagram of PID controller. 

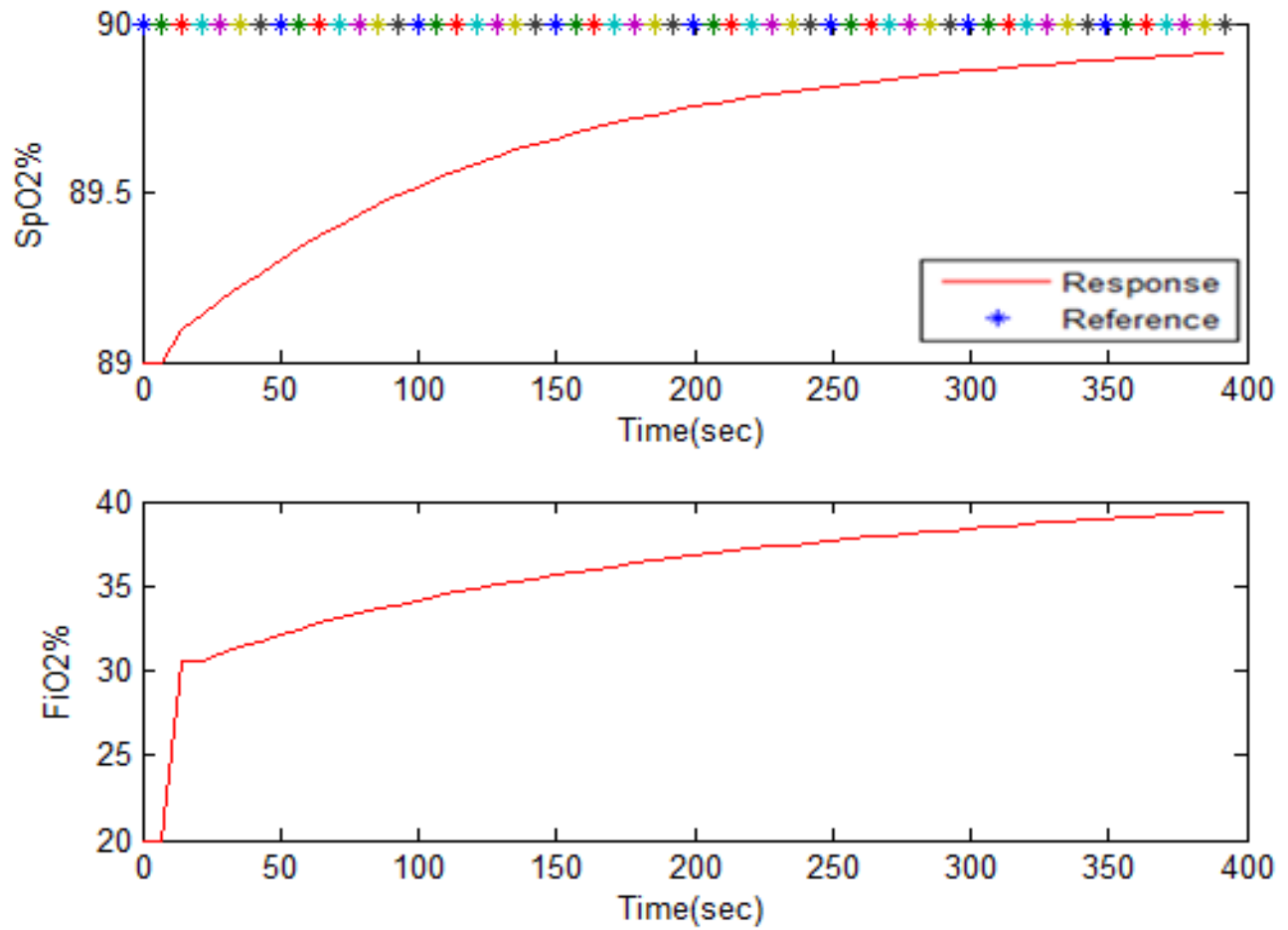

Figure 21. Simulated closed - loop SpO2 with $\mathrm{Kp}=0.1, \mathrm{Ki}=100$, and $\mathrm{Kd}=0.00001$.
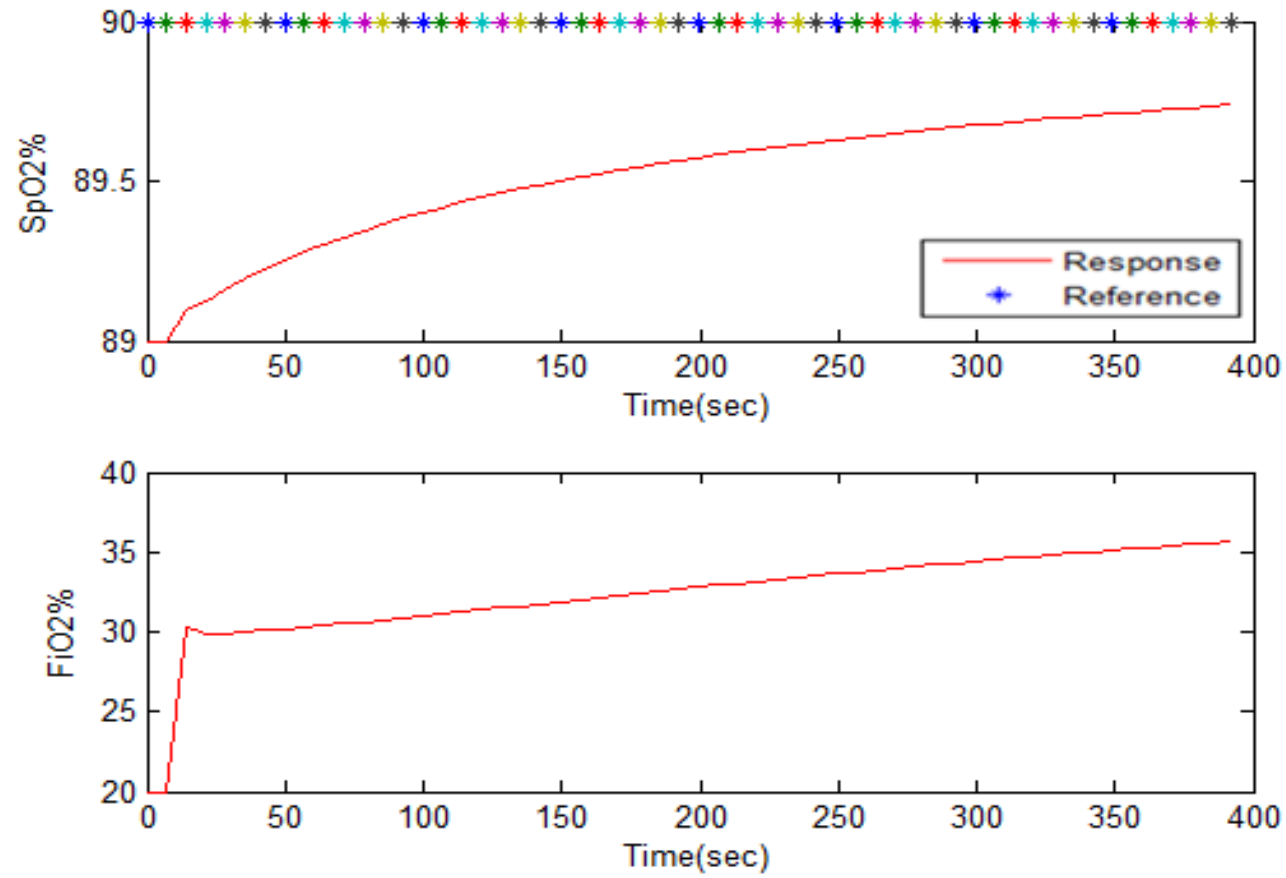

Figure 22. Simulated closed $-\operatorname{loop} \mathrm{SpO} 2$ with $\mathrm{Kp}=0.1, \mathrm{Ki}=50$, and $\mathrm{Kd}=0.00001$. 

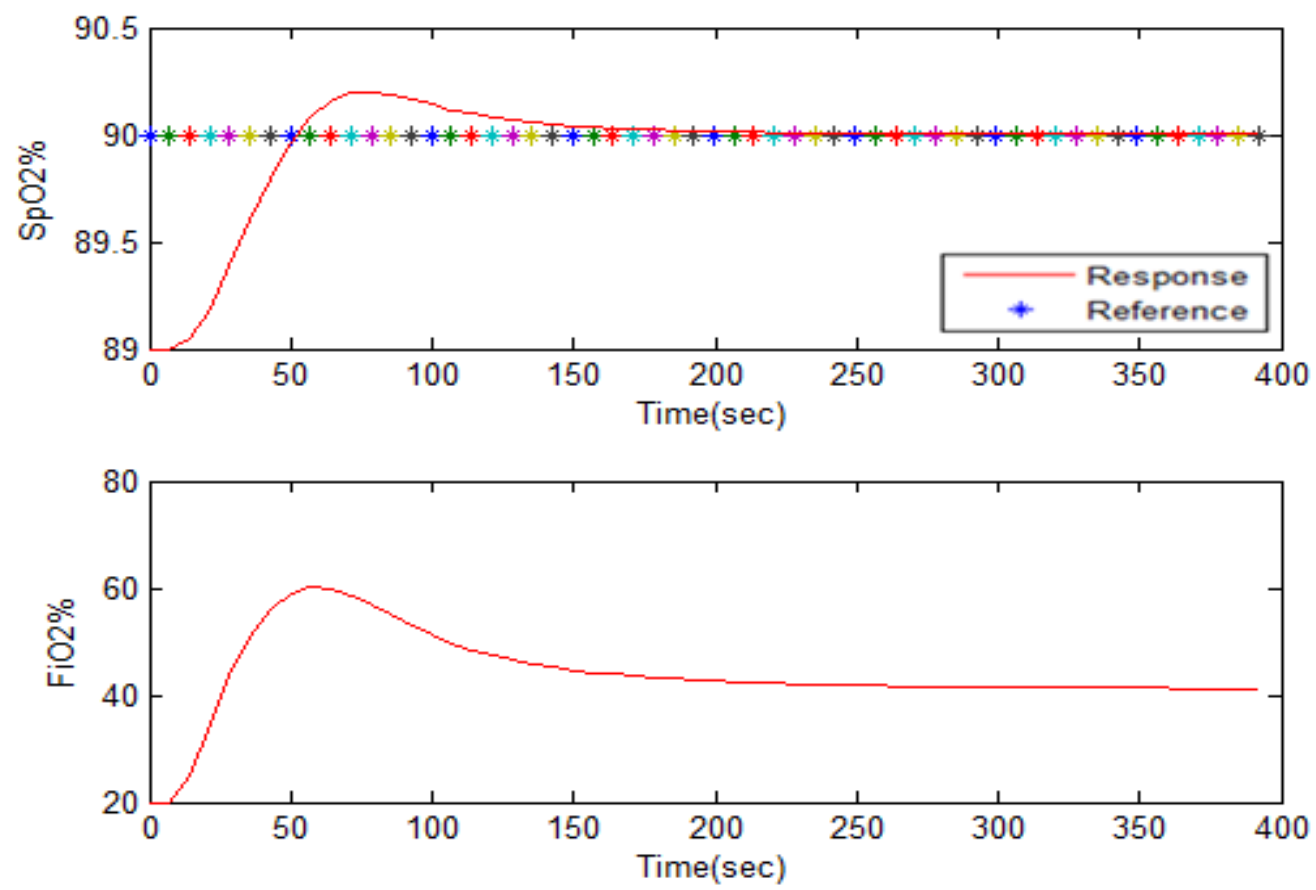

Figure 23. Simulated closed - loop SpO2 with $\mathrm{Kp}=0.00001, \mathrm{Ki}=1000$, and $\mathrm{Kd}=0.00001$.
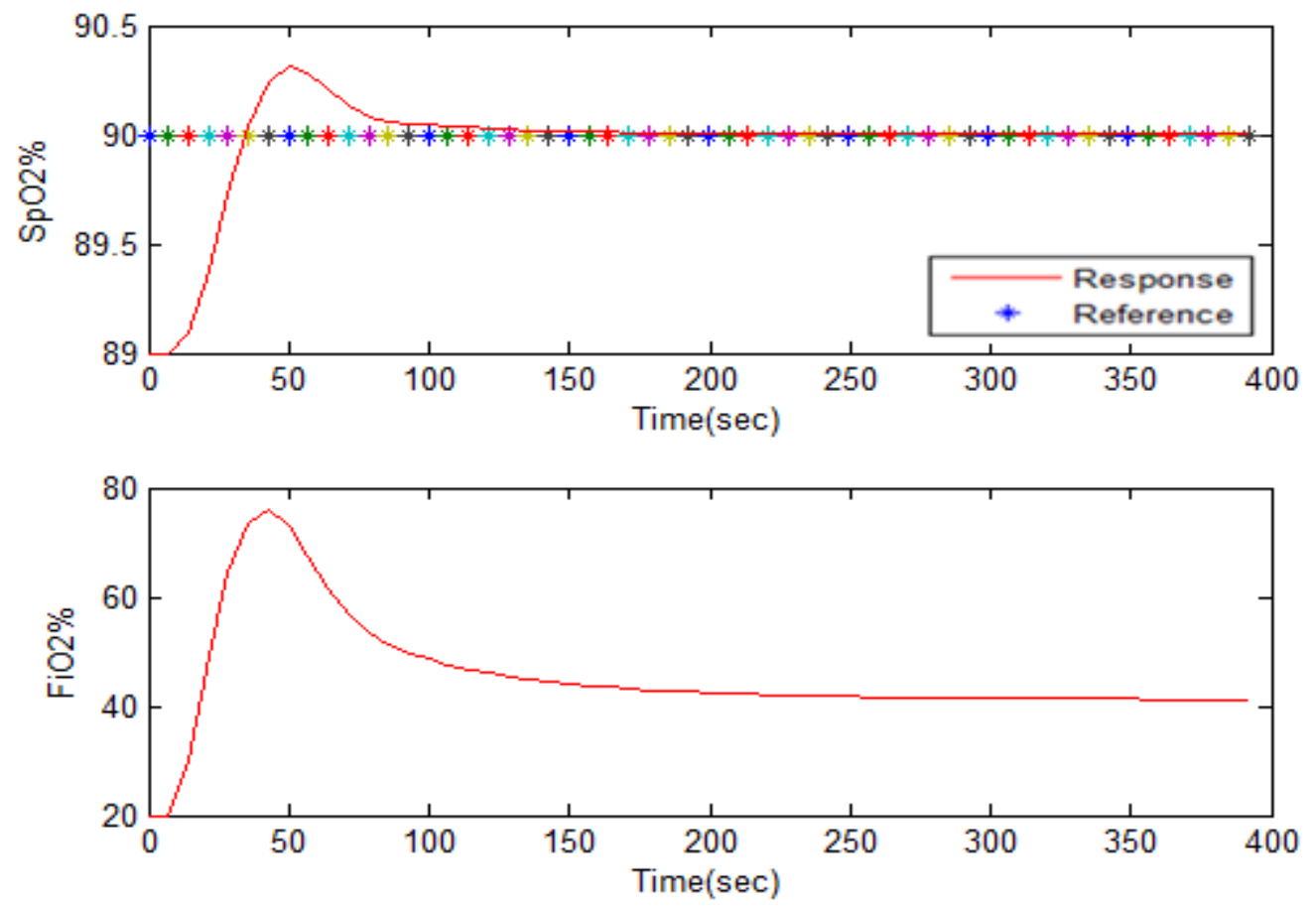

Figure 24. Simulated closed - loop $\mathrm{SpO2}$ with $\mathrm{Kp}=\mathbf{0 . 0 0 0 0 1}, \mathrm{Ki}=\mathbf{2 0 0 0}$, and $\mathrm{Kd}=\mathbf{0 . 0 0 0 0 1}$. 

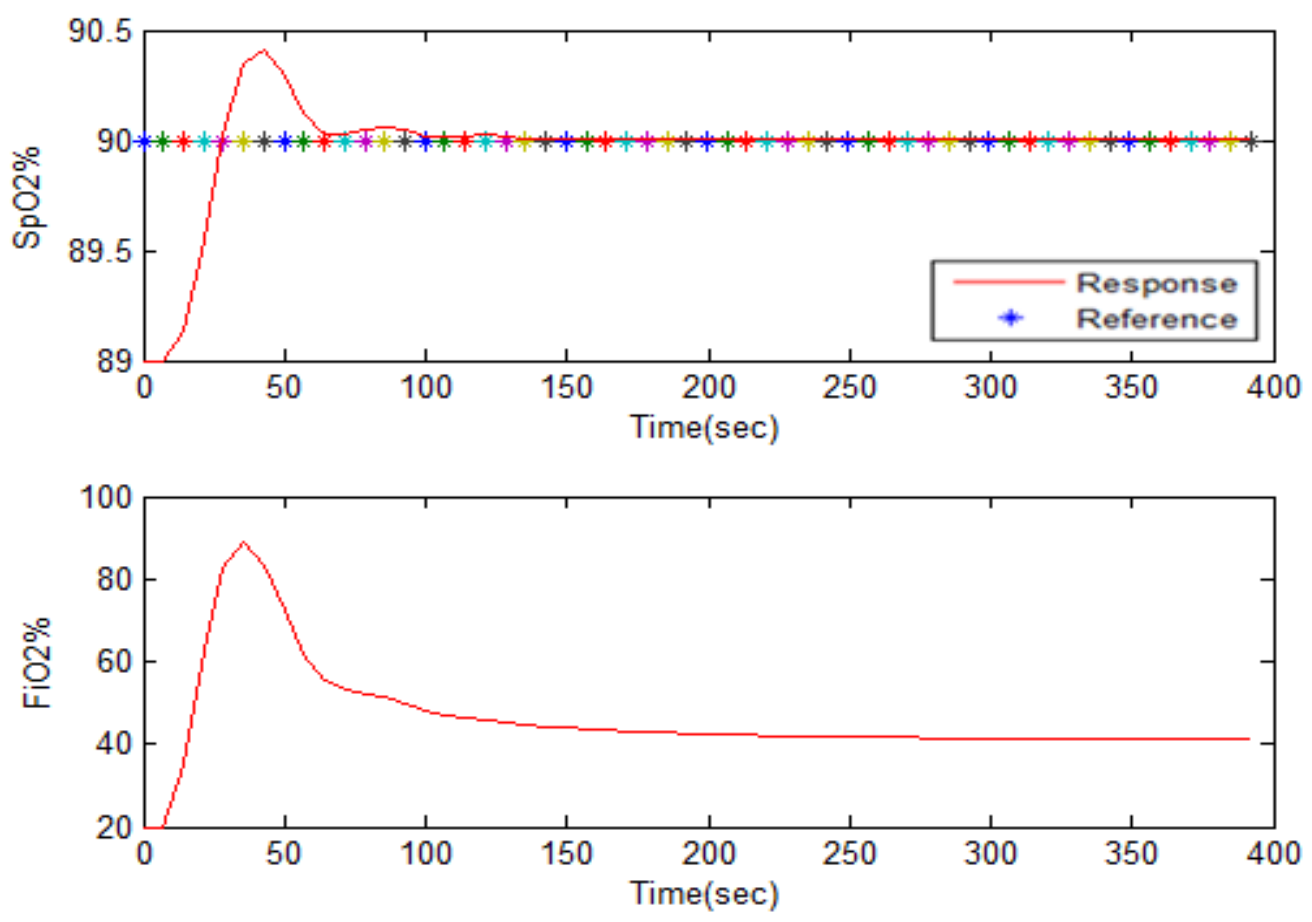

Figure 25. Simulated closed - loop $\mathrm{SpO}$ with $\mathrm{Kp}=0.00001, \mathrm{Ki}=3000$, and $\mathrm{Kd}=0.00001$.
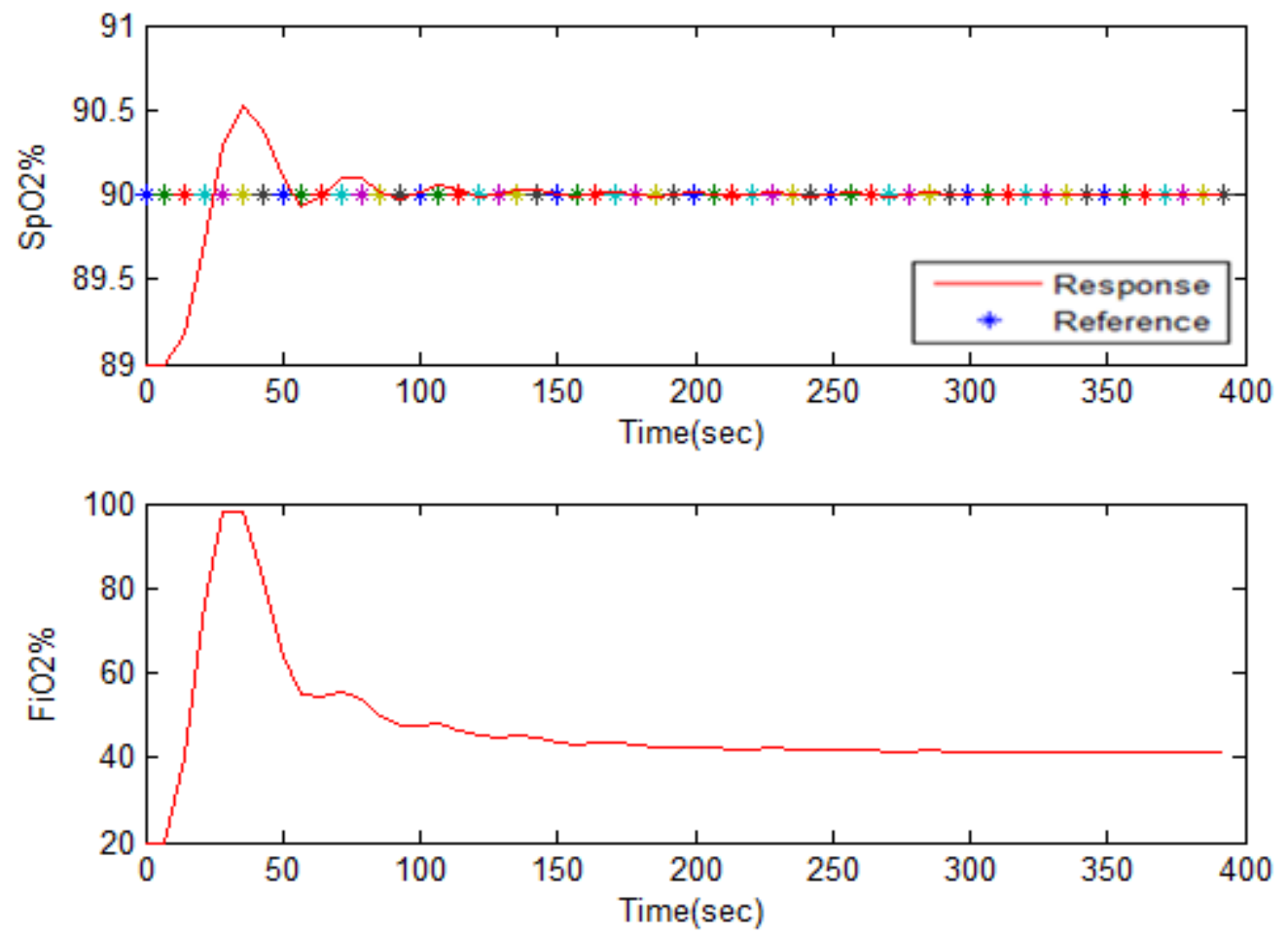

Figure 26. Simulated closed - loop SpO2 with $\mathrm{Kp}=0.00001, \mathrm{Ki}=4000$, and $\mathrm{Kd}=0.00001$. 

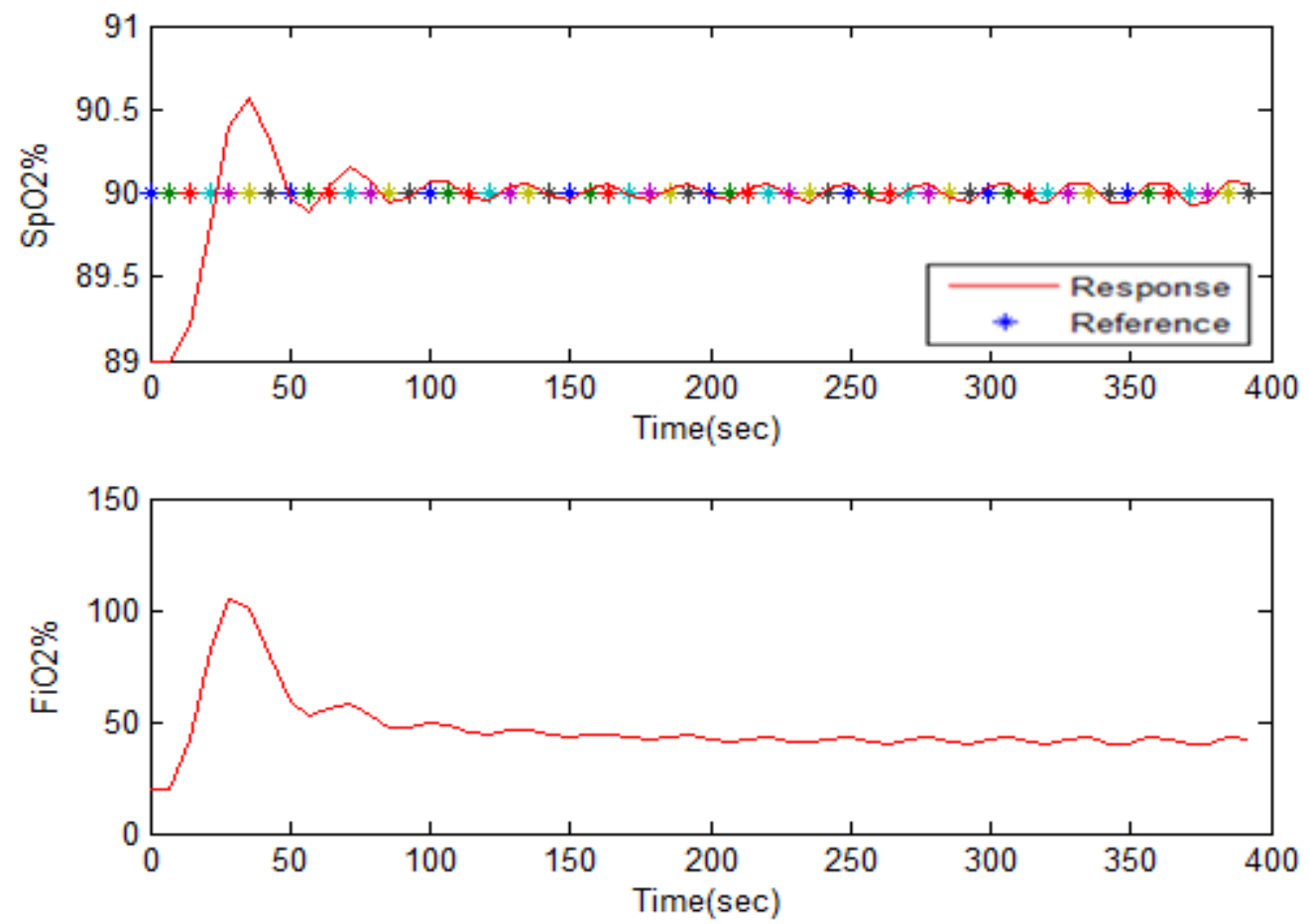

Figure 27. Simulated closed - loop $\mathrm{SpO} 2$ with $\mathrm{Kp}=\mathbf{0 . 0 0 0 0 1}, \mathrm{Ki}=4500$, and $\mathrm{Kd}=0.00001$.
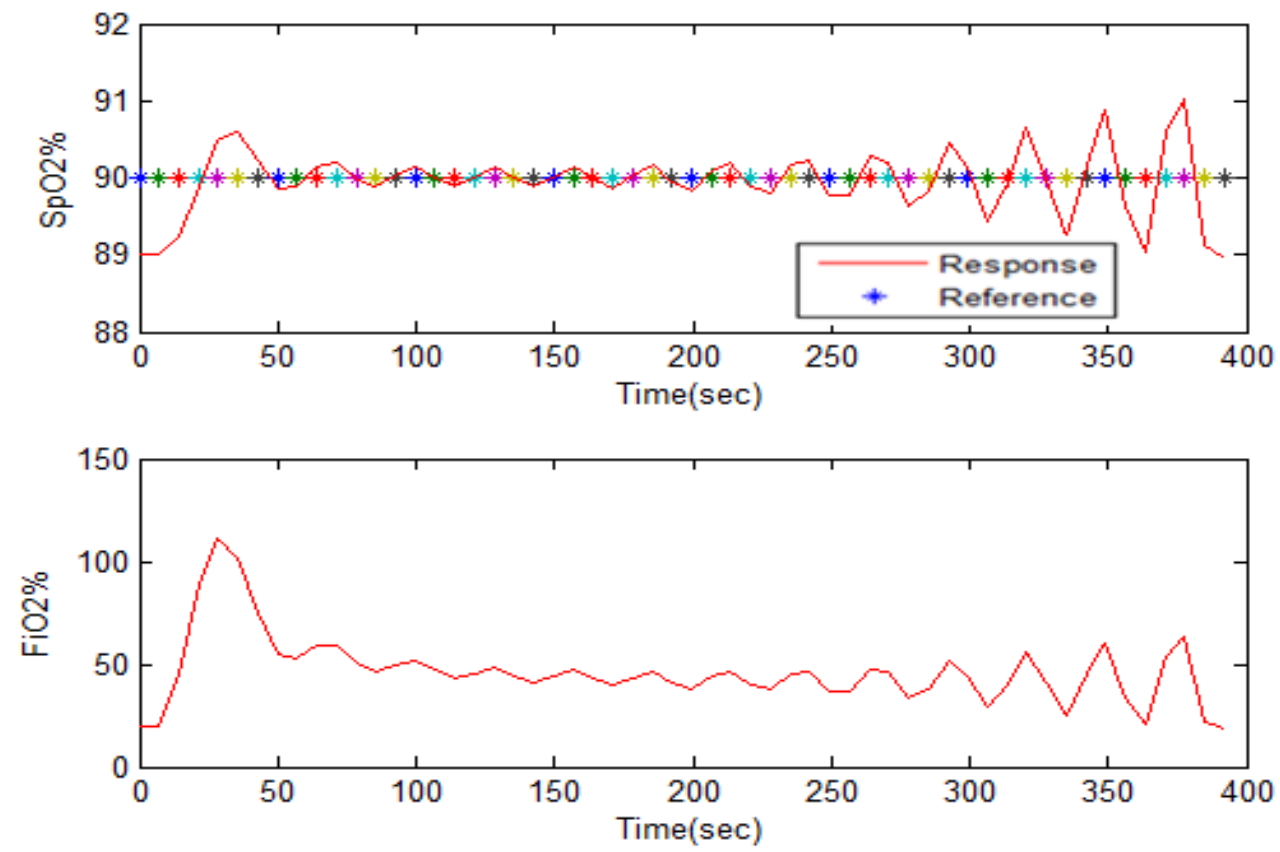

Figure 28. Simulated closed - loop $\mathrm{SpO2}$ with $\mathrm{Kp}=0.00001, \mathrm{Ki}=5000$, and $\mathrm{Kd}=0.00001$. 

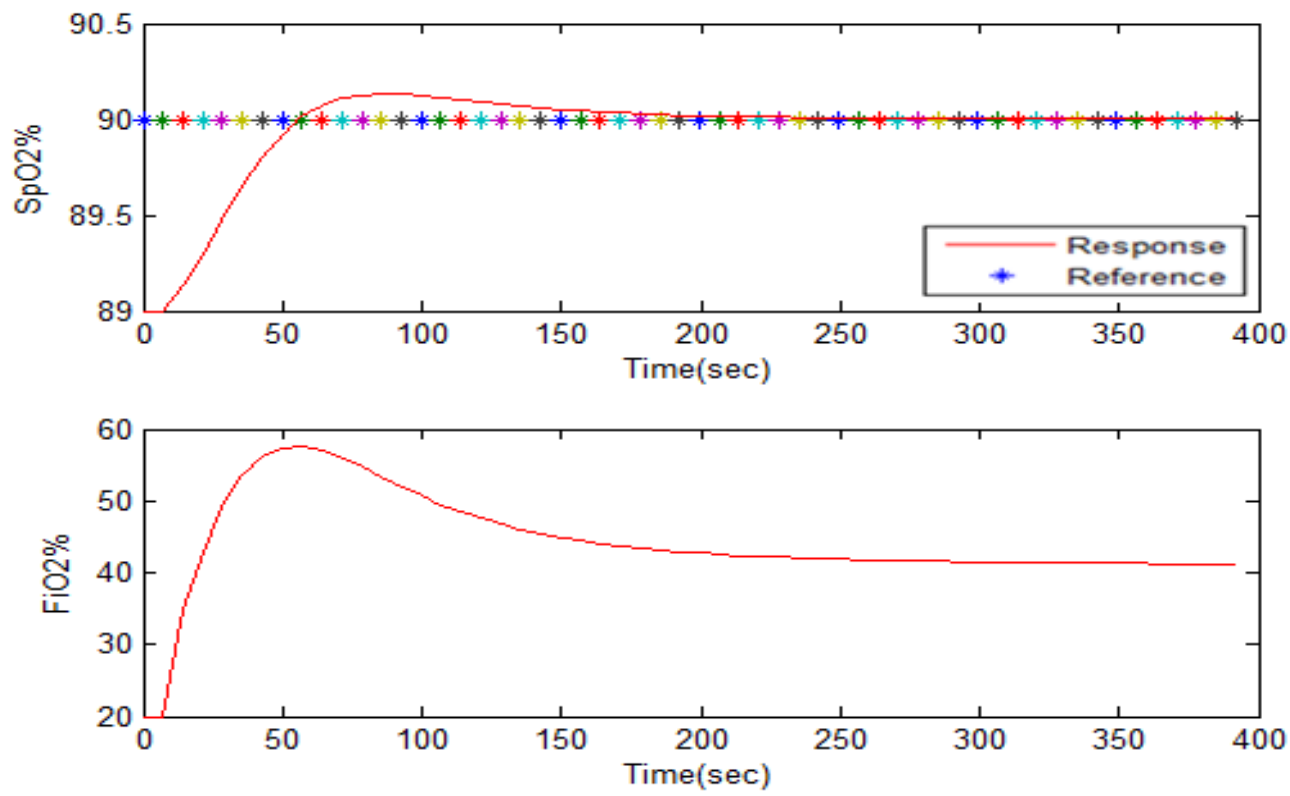

Figure 29. Simulated closed - loop $\mathrm{SpO} 2$ with $\mathrm{Kp}=0.1, \mathrm{Ki}=1000$, and $\mathrm{Kd}=0.00001$.
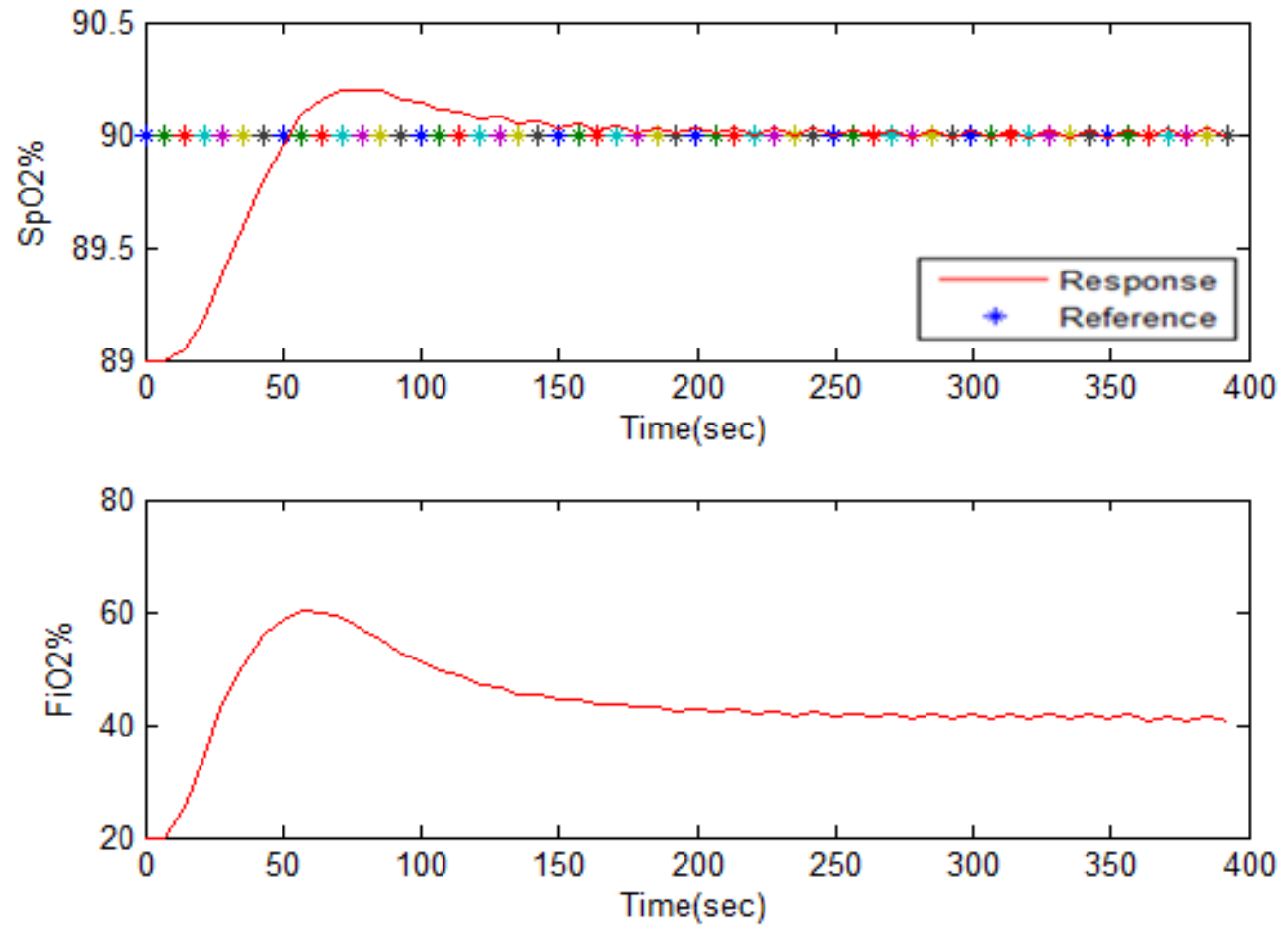

Figure 30. Simulated closed - loop SpO2 with $\mathrm{Kp}=0.001, \mathrm{Ki}=1000$, and $\mathrm{Kd}=0.001$. 

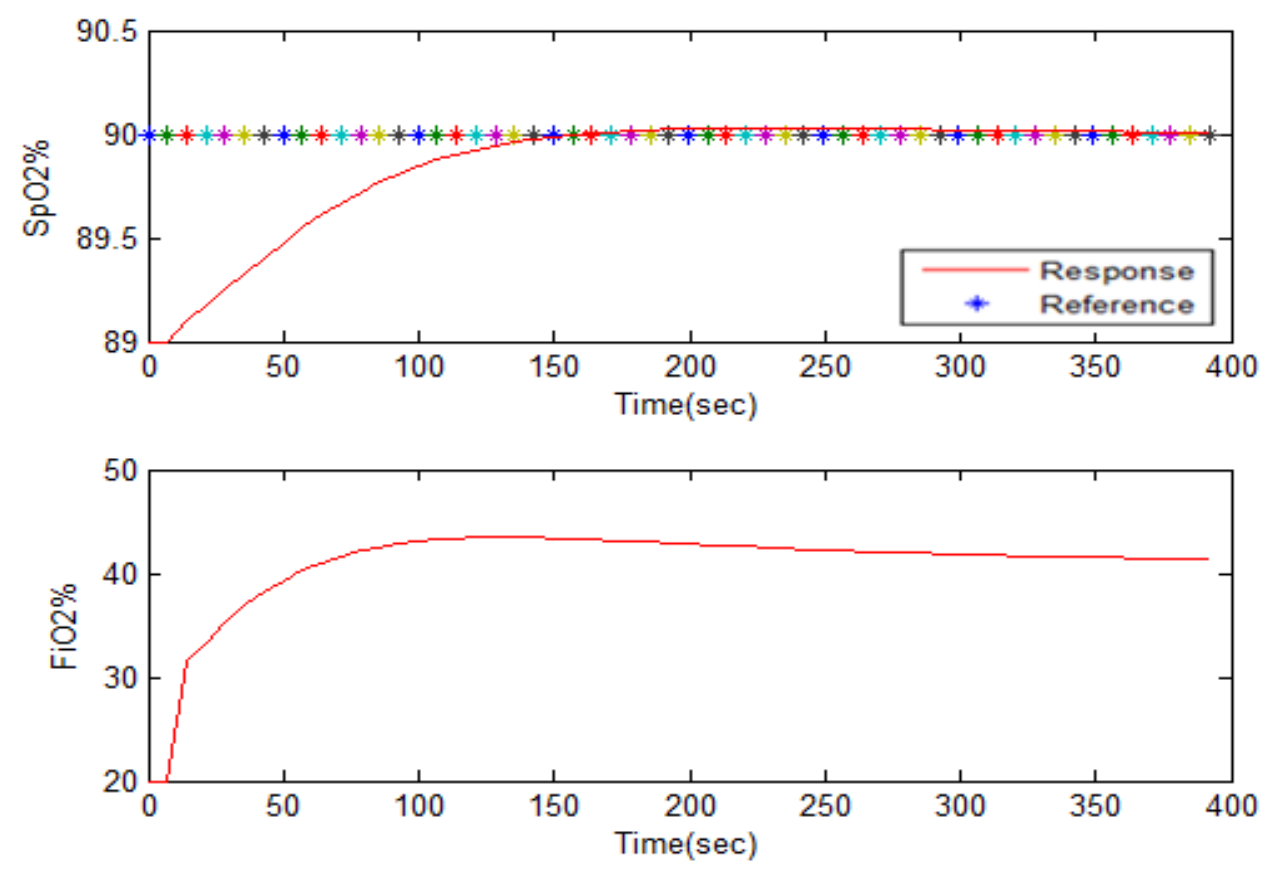

Figure 31. Simulated closed $-\operatorname{loop} \mathrm{SpO2}$ with $\mathrm{Kp}=0.1, \mathrm{Ki}=300$, and $\mathrm{Kd}=0.0001$.

The control action in Eq (3.2) is simulated with tuning $K_{p}, K_{i}$ and $K_{d}$ by trial and error by matlab codes and applied as input $\mathrm{FiO}_{2}$ to the plant to get $\mathrm{SpO}$ with ranging from $85-93 \%$. In this test, the best response is in Fig 16 because there is no steady state error and minimum settling time and the value of $\operatorname{SpO} \mathrm{O}_{2}$ after $170 \mathrm{sec}$. 


\subsection{Model Predictive Control (MPC).}

The main function of Model Predictive Control is to find the input signal that best corresponds to some criteria which predict how the system will behave by applying this signal. Model predictive control (MPC) was initially developed for the control of large constrained systems with slow dynamics and has found application in the process control industries. Model Predictive Control (MPC) is an optimal control strategy based on numerical optimization. Future control inputs and future plant responses are predicted using a system model and optimized at regular intervals with respect to a performance index. Predictive control has become the most widespread advanced control methodology current in use in this industry. MPC has been developed so that stability, optimality, and robustness properties are well understood. Advances in real - time computational abilities are making this approach attractive for a wider range of applications. There are many methods used to introduce a guarantee of stability into the design optimization. The use of an infinite prediction horizon, Model Predictive Control (MPC), also referred to as Receding Horizon Control and moving optimal control, has been widely adapted in industry as an effective means to deal with multivariable constrained control problems [31].

Most control design techniques need a control model of the plant with fixed structure and parameters. If the control model were an exact, rather than an approximate, description of the plant and there were no external disturbances, the 
process could be controlled by an open - loop controller. Feedback is necessary in process control because of the external perturbations and model inaccuracies in all real processed.

The process of robust control is to design a controller which keeps the stability and performance even the models inaccuracies. In order to model the system, the most common techniques are frequency response uncertainty and transfer function parametric uncertainty modeling. Figure 32 shows the block diagram of the basic structure of MPC.

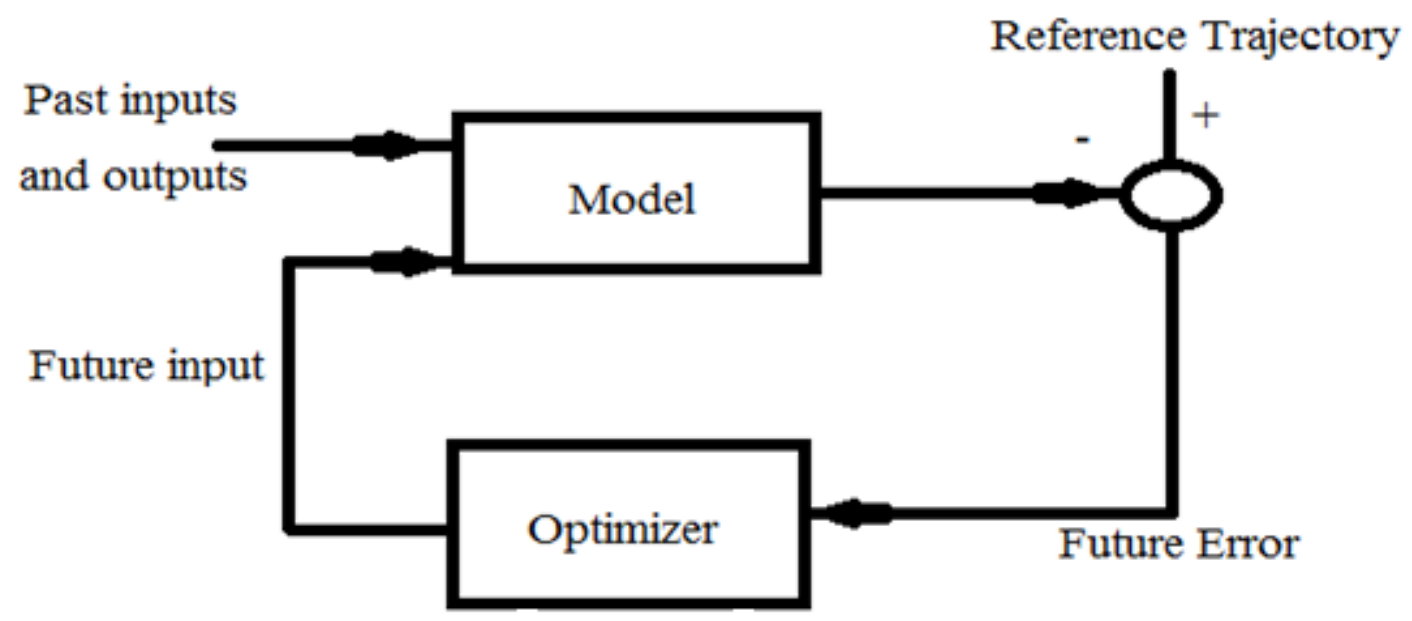

Figure 32. The Basic Structure of MPC. 


\subsubsection{MPC Strategy}

The MPC Strategy is represented as in Figure 33.

1 - The future outputs for a determined horizon $N$, called the prediction horizon, are predicted at each instant $t$ using the process model. All the predicted outputs rely on the known values which are past inputs and outputs and on the future control signals $(t+k), k=0, \ldots \ldots, N-1$.

2 - The Optimizing used to calculate the future control signals in order to keep the process as close as possible to the reference trajectory $w(t+k)$ uses a criterion that usually takes the form of a quadratic function of the error between the predicted output signal and the predicted reference trajectory. An explicit solution can be obtained if the criterion is quadratic, the model is linear and there are no constraints. Otherwise an iterative optimization method has to be used.

3 - The control signal is sent to the process.

For this strategy, a model is used to predict the future plant outputs, based on past and current values and on the proposed optimal future control actions. These actions are calculated by the optimizer taking into account the cost function (where the future tracking error is considered) as well as the constraints.

Transfer function models, are simple and is used in many control design methods, and is valid for many kinds of processes. The state - space model is also used in some formulations. The optimizer is another fundamental part of the strategy as it provides control actions. If the cost is quadratic, its minimum can be 
obtained as an explicit linear function of past inputs and outputs and the future reference trajectory. In the presence of inequality constraints the solution has to be obtained by more computationally taxing numerical algorithms.

In this work, we consider a mathematical system model of recovery from desaturation events developed based on respiratory system. We use the step response model of the respiratory system because it has one input, $\mathrm{FiO}_{2}$ and one output, $\mathrm{SpO}_{2}$, that is developed and completed by $\mathrm{Yu}$.

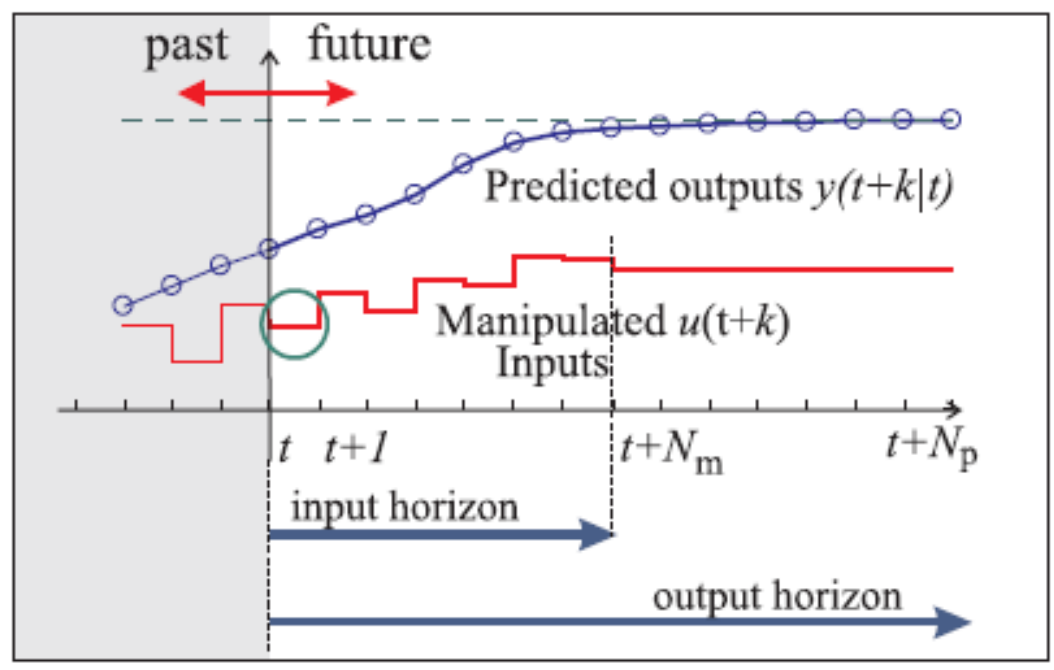

Figure 33. Receding Horizon strategy. 


\subsection{Dynamic Matrix Control}

Dynamic Matrix Control (DMC) was the first model predictive control MPC algorithm and available in almost all commercial industrial distributed control systems. The DMC algorithm includes as one of its major components, a technique to predict the future output of the system as a function of the inputs and disturbances. This prediction capability is necessary to determine the optimal future control inputs.

All the MPC algorithms possess common elements, and different options can be chosen for each one of these elements giving rise to different algorithms. These are three

- Prediction Model

- Objective Function

- Obtaining the control law

The model is the corner - stone of the MPC; a complete design should include the necessary mechanisms for obtaining the best possible model, which should be complete enough to fully capture the process dynamics. The use of the process model is determined by the necessity to calculate the predicted output at future instants $\hat{\mathrm{y}}(t+k)$.

The process model employed in this formulation is the step response of the plant given as 


$$
y(t)=g_{1}(t) \Delta u(t-1)+g_{2}(t) \Delta u(t-2)+g_{3}(t) \Delta u(t-3)+. .
$$

where $g_{i}$ are the sampled output values for the step input and $\Delta u(t)=u(t)-$ $u(t-1)$ and $N$ is usually a high value for prediction.

As an impulse can be considered as the difference between two steps with a lag of one sampling period, it can be written for a linear system that:

$$
h_{i}=g_{i}-g_{i-1} \quad g_{i}=\sum_{j=1}^{i} h_{j}
$$

where $h_{i}$ is the sampled output when the process is excited by a unit impulse.

Now the predictions can be computed along the prediction horizon $(k=$ $1, \ldots \ldots \ldots, p)$, considering $m$ control actions.

$$
\begin{gathered}
\hat{\mathrm{y}}(t+p)=g_{1}(t) \Delta u(t+p-1)+g_{2}(t)+\Delta u(t+p-2)+g_{3}(t)+ \\
\Delta u(t+p-3)+\ldots(3.4)
\end{gathered}
$$

Define the system's dynamic matrix $G$ as:

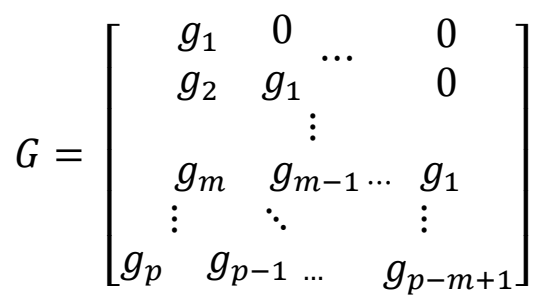


and it can be written that $\hat{\mathrm{y}}=G u$.

Observe that $G$ is made up of $m$ (the control horizon) columns of the system's step response appropriately shifted down in order.

- $\quad \hat{\mathrm{y}}$ is a $\mathrm{p}-$ dimensional vector containing the system prediction along the horizon.

- $\quad u$ represents the $\mathrm{m}-$ dimensional vector of control increments.

- This is the expression that relates the future outputs with the control increments.

In the objective function, plant operation requirements determine the performance criteria of the control system. These criteria must be expressed in mathematical terms so that a control law can be obtained in algorithmic form. In DMC, a quadratic objective function is used which can be stated in its simplest form as

$$
\min _{\Delta u(k) \ldots . . . \Delta \mathrm{u}(\mathrm{k}+\mathrm{m}-1)} \sum_{l=1}^{p}\|y([y(k+l \mid k)-r(k+l)])\|^{2}
$$

This criterion minimizes the sum of squared deviations of the predicted CV values from a time - varying reference trajectory or set point $r(k+l)$ over $p$ 
future time steps. The quadratic criterion penalizes large deviations proportionally more than smaller ones, so that on the average the output remains close to its reference trajectory and large excursions are avoided.

where $m \leq p$ always. This means that DMC determines the next $m$ moves only. The choices of $m$ and $p$ affect the closed - loop behavior. Moreover, $m$, the number of degrees of freedom, has a dominant influence on the computational effort.

Due to inherent process interactions, it is generally not possible to keep all outputs close to their corresponding reference trajectories simultaneously. Therefore, in practice only a subset of the outputs is controlled well at the expense of larger excursion in others. This can be influenced transparently by including weights in the objective function as follows:

$$
\min _{\Delta u(k) \ldots . . . \Delta \mathrm{u}(\mathrm{k}+\mathrm{m}-1)} \sum_{l=1}^{p}\left\|\Gamma_{l}^{y}([y(k+l \mid k)-r(k+l)])\right\|^{2}
$$

If a system with two outputs $y_{1}$ and $y_{2}$, and constant diagonal weight matrices of the form

$$
\Gamma_{l}^{y}=\left[\begin{array}{cc}
l_{1} & 0 \\
0 & l_{2}
\end{array}\right]
$$


The objective becomes

$$
\begin{gathered}
\min _{\Delta u(k) \ldots . . . \Delta \mathrm{u}(\mathrm{k}+\mathrm{m}-1)}\left\{l_{1}^{2} \sum_{l=1}^{p}\left[\left(\left[y_{1}(k+l \mid k)-r_{1}(k+l)\right]\right)\right]^{2}+\right. \\
\left\{l_{2}^{2} \sum_{l=1}^{p}\left[\left(\left[y_{2}(k+l \mid k)-r_{2}(k+l)\right]\right)\right]^{2}\right.
\end{gathered}
$$

Thus, the larger the weight is for a particular output, the larger is the contribution of its sum of squared deviations to the objective. This will make the controller bring the corresponding output closer to its reference trajectory.

Finally, the manipulated variable moves that make the output follow a given trajectory could be too severe to be acceptable in practice. This can be corrected by adding a penalty term for the manipulated variable moves to the objectives as the following:

$$
\min _{\Delta u(k)} \sum_{l=1}^{p}\left\|\Gamma_{l}^{y}[([y(k+l \mid k)-r(k+l)])]\right\|^{2}+\sum_{l=1}^{m} \| \Gamma_{l}^{u}\left[\Delta_{u}(k+l-1] \|^{2}\right.
$$

Note that the larger the elements of the matrix $\Gamma_{l}^{u}$ are the smallest the resulting moves will be, and consequently, the output trajectories will not be followed as closely. Thus, the relative magnitude of $\Gamma_{l}^{y}$ and $\Gamma_{l}^{u}$ will determine the trade off between following the trajectory closely and reducing the action of the manipulated variables. 
For any assumed set of present and future control moves $\Delta u(t), \Delta u(t+$ $1), \ldots \ldots, \Delta u(t+m-1)$ then the future behavior of the process outputs $y(t+$ 1), $y, \ldots, y(k+p \mid k)$ can be predicted over a horizon $P$. The $m$ present and future control moves $(m \leq p)$ are computed to minimize a quadratic objective of the form as in $\mathrm{Eq}$ (3.6).

where $\Gamma_{l}^{y}$ and $\Gamma_{l}^{u}$ are weighting matrices to penalize particular components of $y$ or $u$ at certain future time intervals. $r(k+1)$ is the vector of future reference values (set point). At the first sampling, the $m$ control moves and $\Delta u(k)$ is implemented. At the next sampling interval, new values of the measured output are obtained, the control horizon is shifted forward by one step, and the same computations are repeated. The moving horizon is leading to get the control law, the feedback control law is

$$
\Delta u(k)=K_{M P C} E_{p}(k+1 \mid k)
$$

where $E_{p}(k+1 \mid k)$ is the vector of predicted future errors over the horizon $P$ which would result if all present and future manipulated variable moves were equal to zero $\Delta u(k)=\Delta u(k+1)=\cdots=0$.

The nominal stability of the closed - loop system in the open - loop stable plants depends only on $K_{M P C}$ which depends on the values of horizon $p$ and, the number of $m$ and the weighting matrices $\Gamma_{l}^{y}$ and $\Gamma_{l}^{u}$. The value of $\Gamma_{l}^{u}$ is used as a tuning parameter which means that increasing $\Gamma_{l}^{u}$ always has the effect of making the control action less aggressive. 
The objective of a DMC controller is to drive the output as close to the set point as possible in a least -squares sense with the possibility of the inclusion of a penalty term on the input moves. Disturbances and modeling errors may lead to deviations between the predicted behavior and actual observed behavior so that the computed manipulated variable moves are actually implemented. The DMC algorithm includes as one of its major components a technique to predict the future output of the system as a function of the inputs and disturbances. The prediction is necessary to determine the optimal future control input.

The optimization problem with a quadratic objective and linear inequalities, which it has defined is a Quadratic Program. By converting to the standard QP formulation the DMC problem becomes:

$$
\min _{\Delta u(k)} \Delta u(k)^{T} H^{u} \Delta u(k)-g(k+1)^{T} \Delta u(k)
$$

where the Hessian of the QP is

$$
H^{u}=D^{T} \Gamma_{l}^{y^{T}} \Gamma_{l}^{y} D+\Gamma_{l}^{u T} \Gamma_{l}^{u}
$$

and the gradient vector is

$$
\mathrm{g}(\mathrm{k}+1)=2 D^{T} \Gamma_{l}^{y^{T}} \Gamma_{l}^{y} E_{p}(k+1)
$$


The DMC algorithm is implemented on - line as follow.

1. Preparation. Do not very the manipulated variables for at least $\mathrm{n}$ time intervals $\Delta u(-1)=\Delta u(-2)=\cdots=\Delta u(-n)=0$.

2. Initialization $(\mathrm{k}=0)$. Measure the output $\hat{y}(0)$ and initialize the model prediction vector.

3. State Update: Set $k=k+1$. Then, update the state according to

$$
\tilde{Y}(k)=M \cdot \tilde{Y}(k-1)+D \Delta u(k-1)
$$

where the first element of $\tilde{Y}(k)$, is the model prediction of the output at time $\mathrm{k}$.

4. Compute the reference trajectory error vector

$$
E_{p}(k+1)=R(k+1)-M \tilde{Y}(k)+I_{p}\left(y_{m}(k)-\tilde{y}(k)\right)
$$

5. Compute the QP gradient vector

$$
g(k+1)=D^{T} \Gamma_{l}^{y^{T}} \Gamma_{l}^{y} E_{p}(k+1)
$$

6. Solve the QP

$$
\min _{\Delta u(k)} \Delta u(k)^{T} H^{u} \Delta u(k)-g(k+1)^{T} \Delta u(k)
$$

7. Go to 3 .

In a moving horizon framework that $\mathrm{QP}$ in (3.13) is solved at each controller execution time after a new prediction is obtained. The only time varying elements 
in this problem are the vectors $E_{p}(k+1)$ (or equivalently $\mathrm{g}(\mathrm{k}+1)$ ). That is, the Hessian $H$ of the QP remains constant for all executions. In that case, a parametric QP algorithm which employs the pre-inverted Hessian in its computations is preferable in order to reduce on-line computation effort. Of course, in case either $\Gamma_{l}^{y}$ or $\Gamma_{l}^{u}$ (or the step response coefficients) need to be updated, or the model's step response coefficients have changed, the Hessian must be recomputed and inverted in background mode in order not to increase the on - line computational requirements. 


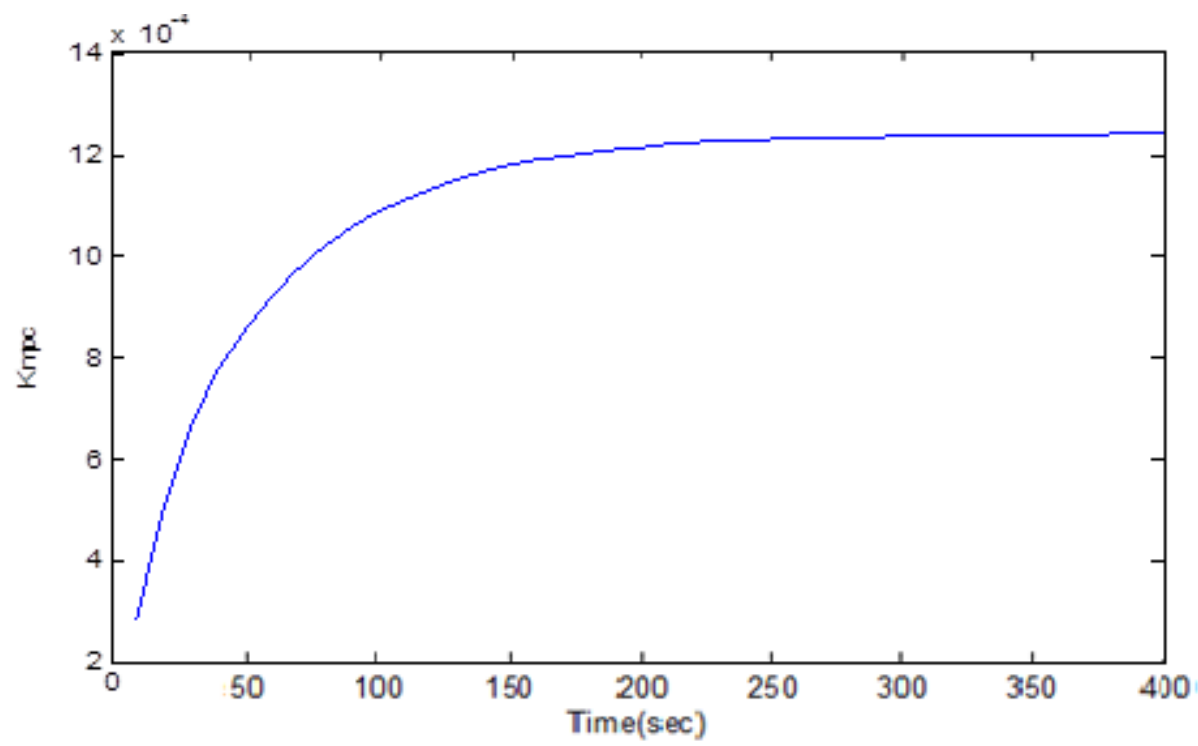

Figure 34. Gain Kmpc of the closed loop system at $\Gamma_{y}=1, \Gamma_{u}=10$.

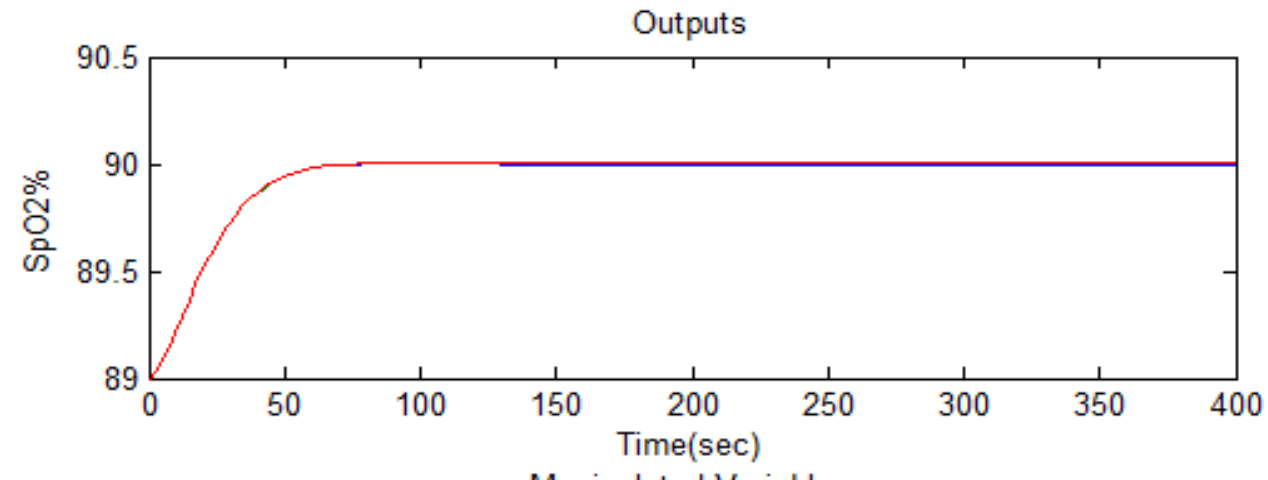

Manipulated Variables

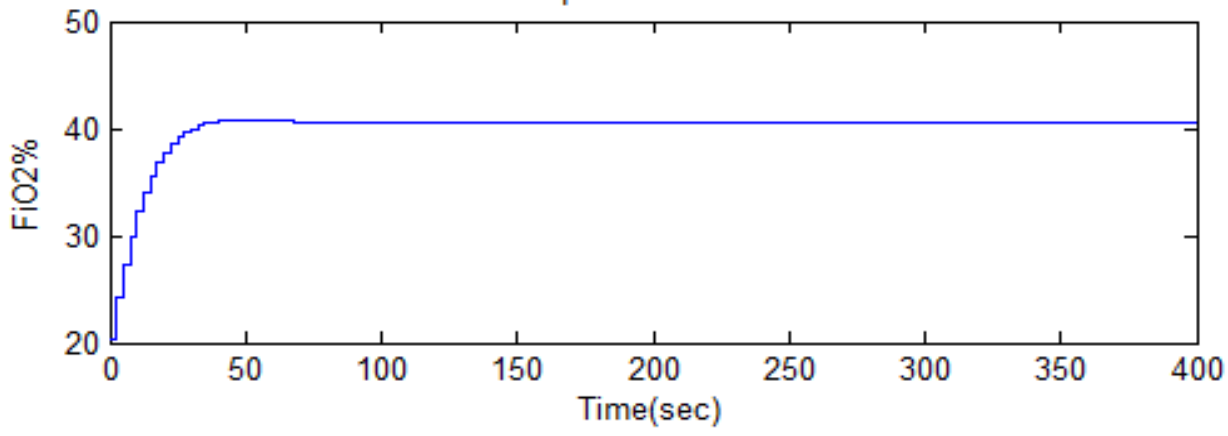

Figure 34. b) Output SpO2 with manipulated variable at $\Gamma_{y}=1, \Gamma_{u}=10$. 


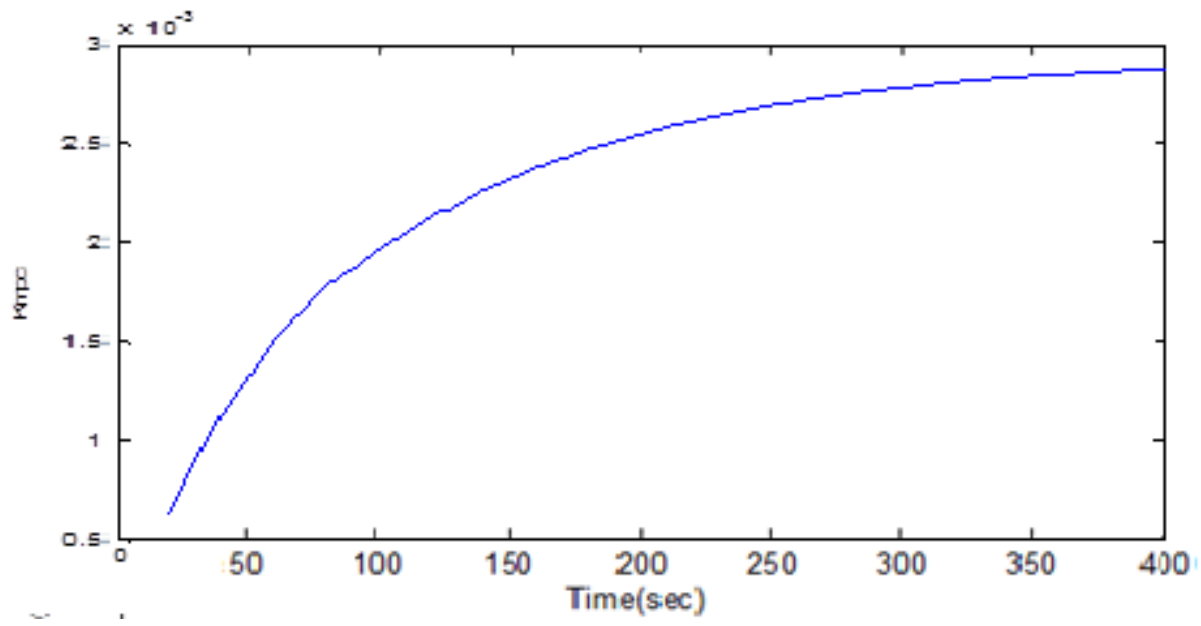

Figure 35. a) Gain Kmpc of the closed loop system at $\Gamma_{y}=1, \Gamma_{u}=20$.
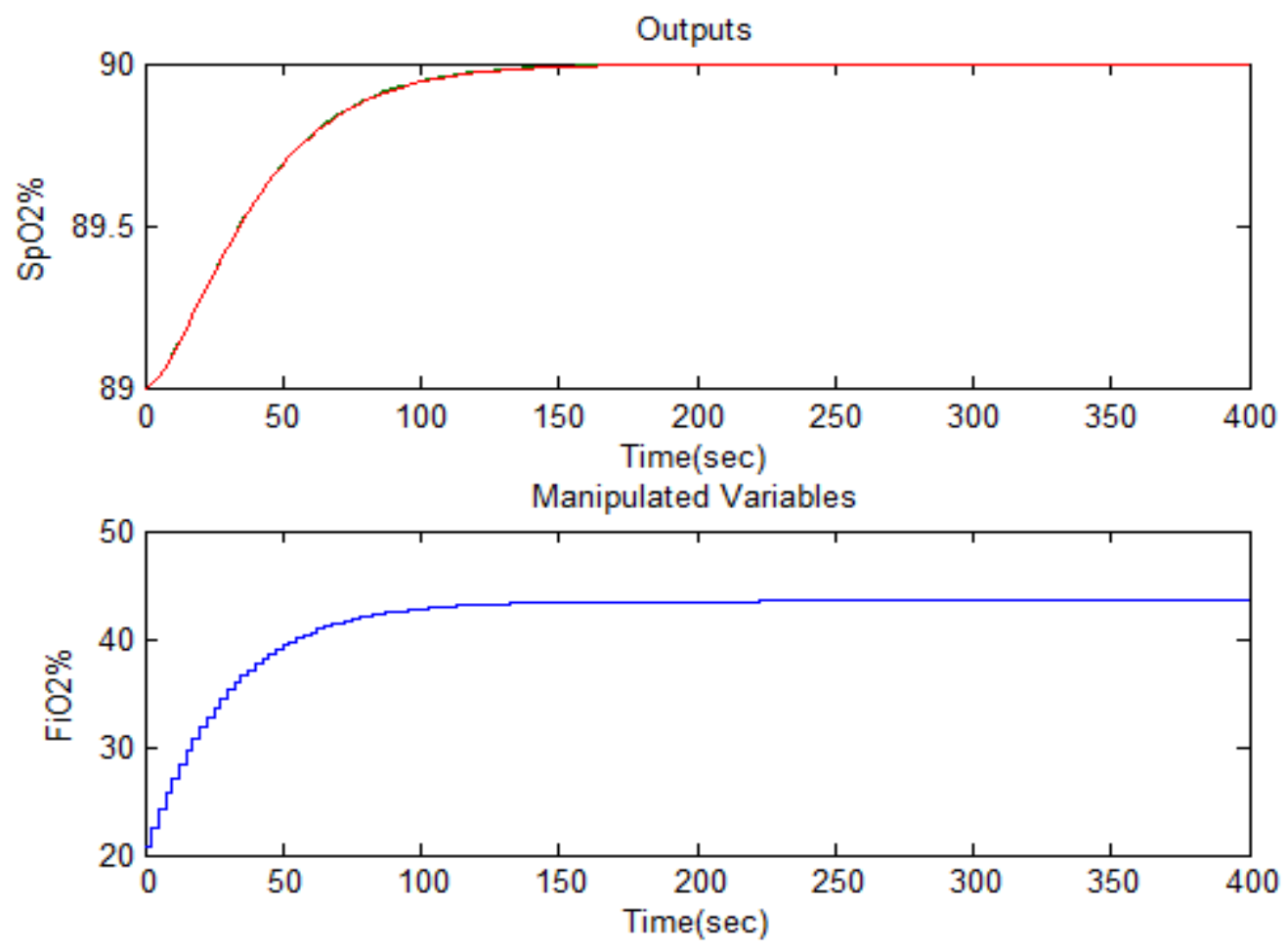

Figure 35. b) Output $\mathrm{SpO} 2$ with manipulated variable at $\Gamma_{y}=1, \Gamma_{u}=20$. 


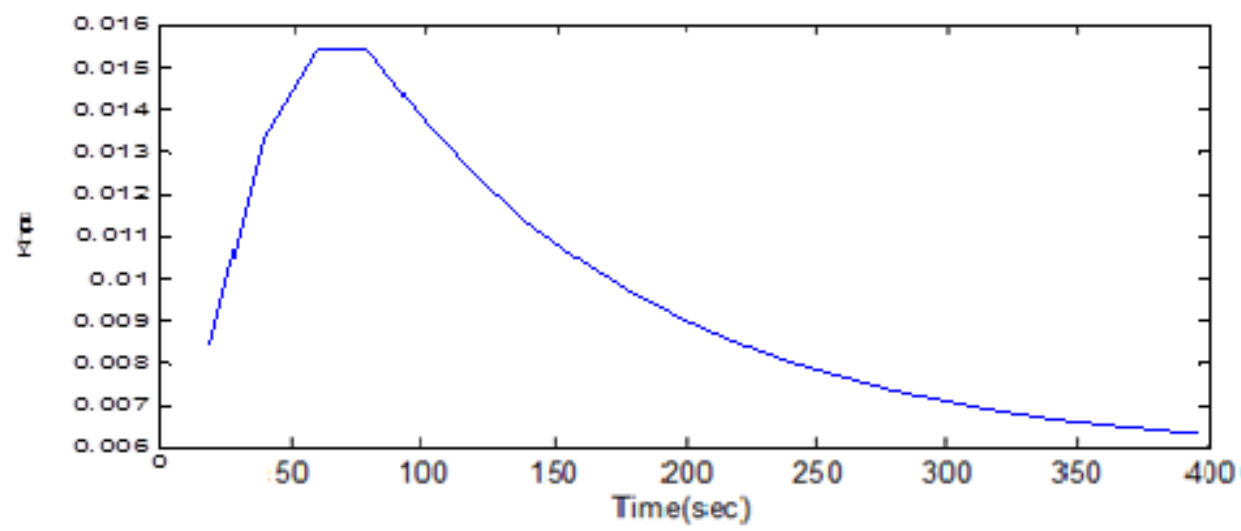

Figure 36. a) Gain Kmpc of the closed loop system at $\Gamma_{y}=1, \Gamma_{u}=5$.
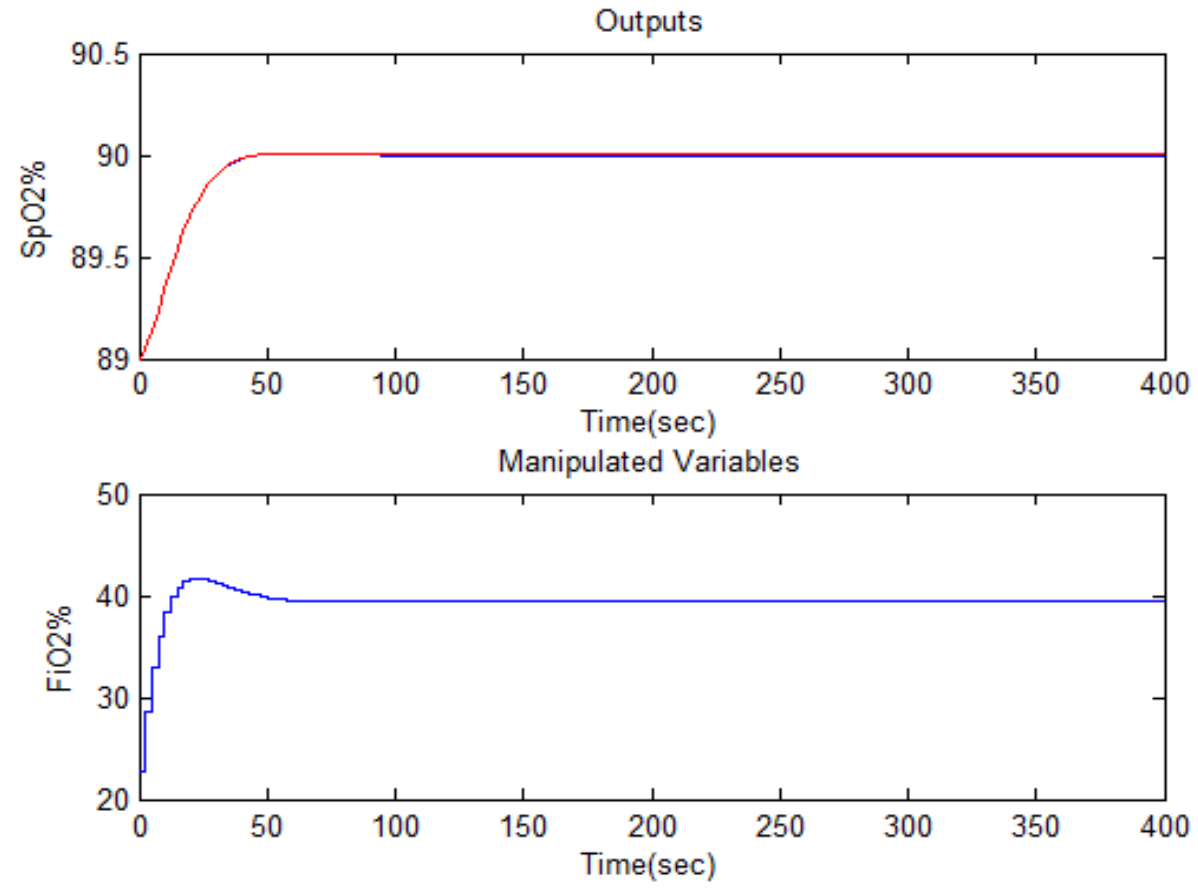

Figure 36. b) Output SpO2 with manipulated variable at $\Gamma_{y}=1, \Gamma_{u}=5$ 


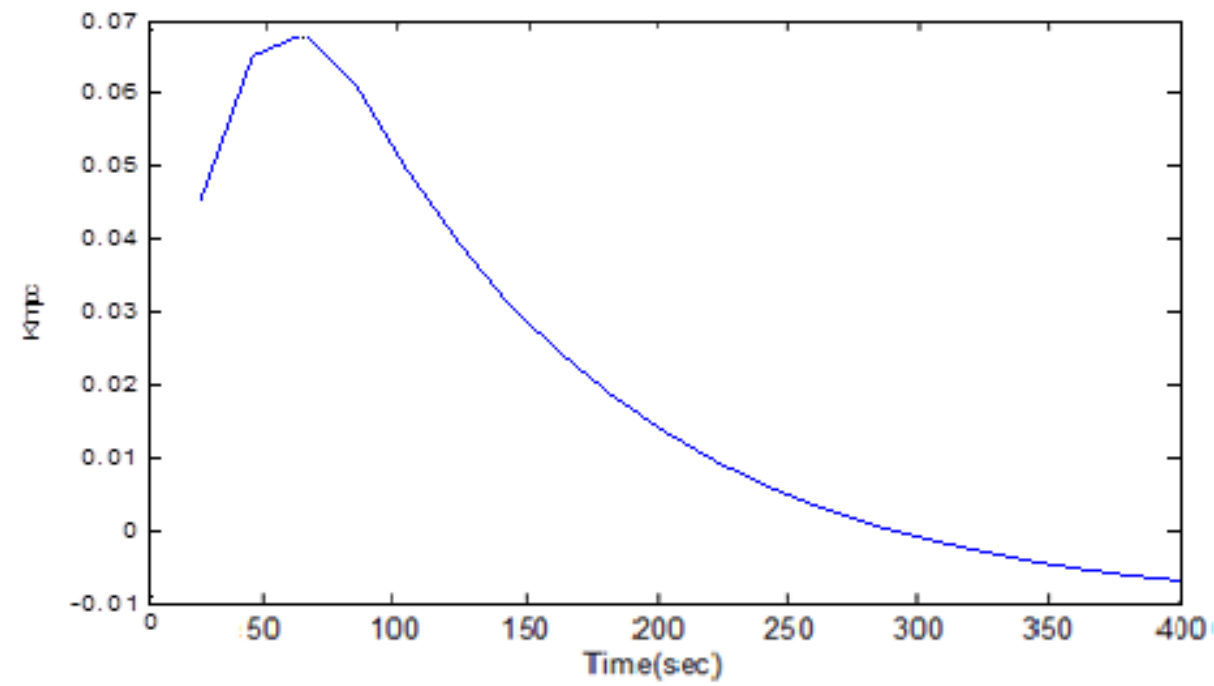

Figure 37. a) Gain Kmpc of the closed loop system at $\Gamma_{y}=1, \Gamma_{u}=2$
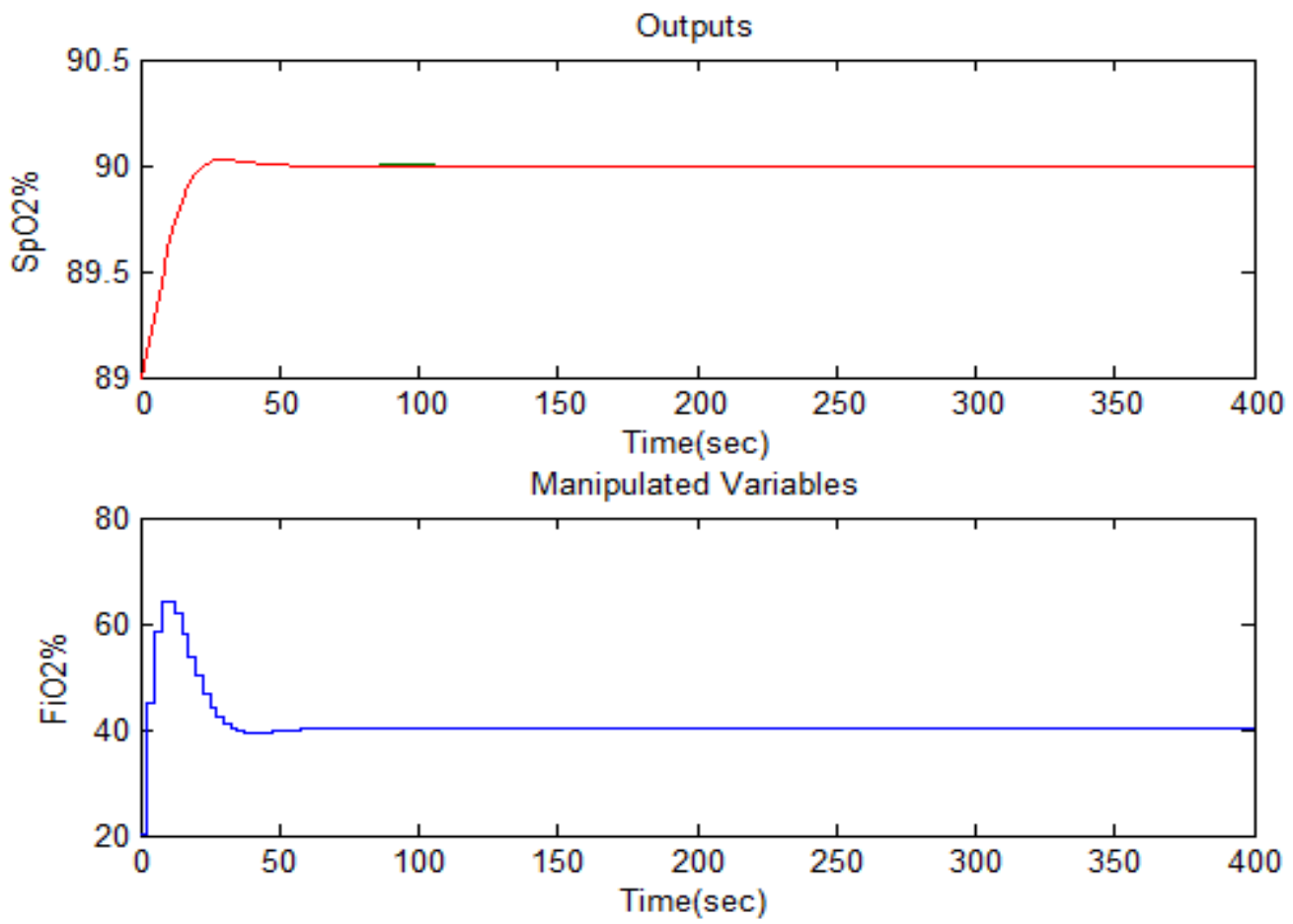

Figure 37. b) Output $\mathrm{SpO} 2$ with manipulated variable at $\Gamma_{y}=1, \Gamma_{u}=2$ 
Not that the larger the elements of the matrix $\Gamma_{l}^{u}$ were, the smaller the resulting moves will be, and consequently, the output trajectories didn't follow as closely. Therefore, the relative magnitude of $\Gamma_{l}^{y}$ and $\Gamma_{l}^{u}$ determined the trade - off between the trajectory closely and reduced the action of the manipulated variable. The best response is in Fig (34) because there is no steady state error and minimum settling time and the value of $\mathrm{SpO}_{2}$ after $65 \mathrm{sec}$. 


\section{Chapter 4: Robustness}

\subsection{Robust Control Oriented Modeling.}

The first step in robust control oriented modeling is to get a model of the plant uncertainty using knowledge of the likely range of parameter variations. The system gain and time constant parameter ranged were shown in [30] the gain was from 1.6 to 6 and time constant from 0.1 to 200 . The ranges of parameters are found from transfer function model obtained by Krone. In order to account for perturbation in the system parameters, a multiplicative uncertainty transfer function weight, $W_{I}$, was added to the system. The multiplicative uncertainty error is defined as

$$
E_{I}(s)=\frac{G_{p}(s)-G_{n o m}(s)}{G_{\text {nom }}(s)}
$$

where $G_{n o m}$ is the nominal plant and $G_{p}$ is the perturbation plant from the nominal. The nominal plant had a system gain of 1.6 and a time constant of 0.5561 seconds. The $G_{\text {nom }}$ was calculated by gain uncertainty multiplied the nominal plant. The line solid plotted in Fig 38.b is the $W_{I}$ uncertainty weight bounding the maximum error for all values of frequencies by plotting the multiplicative uncertainty transfer function, and we can see that in Figures 38.a and 38.b. A block diagram with uncertainty can be seen in Figure 39. The transfer function for the multiplicative uncertainty is 


$$
w_{I}=\frac{0.8 s+0.2}{s+0.375}
$$

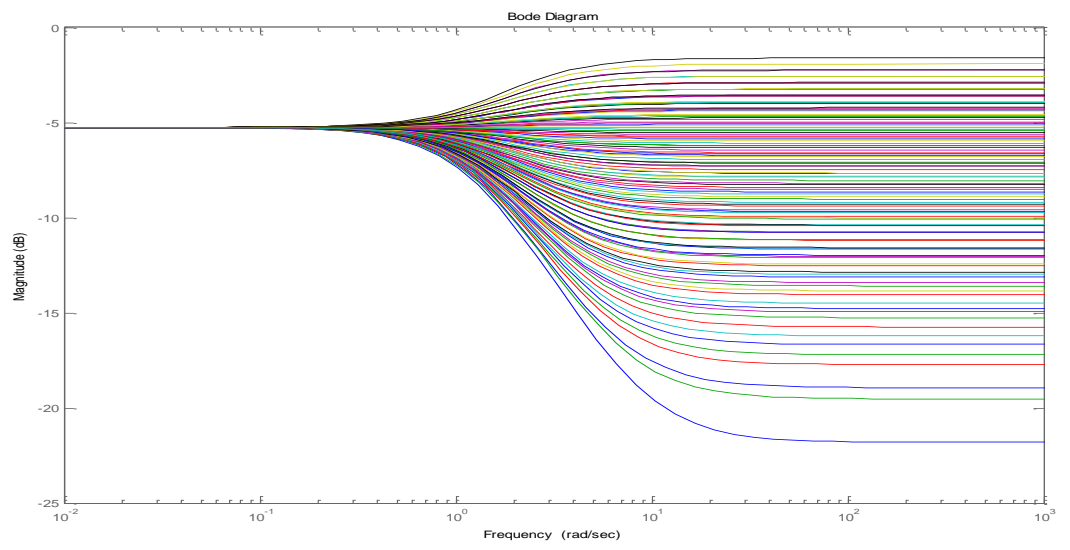

(a)

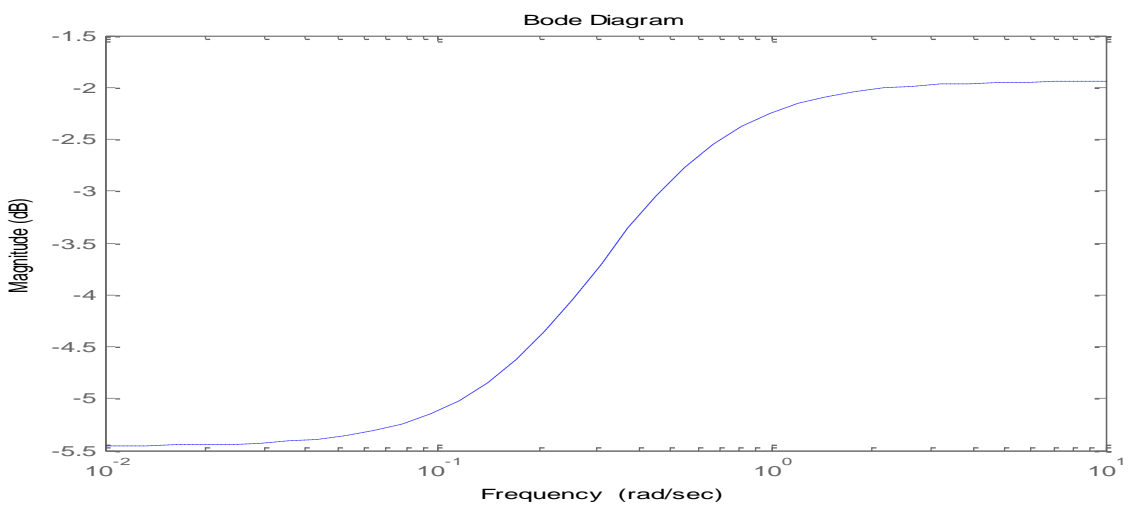

(b)

Figure 38. (a) Multiplicative uncertainty transfer function bounding the maximum error for the set parameter range

(b) Bode plot for transfer function of $w_{I}$. 


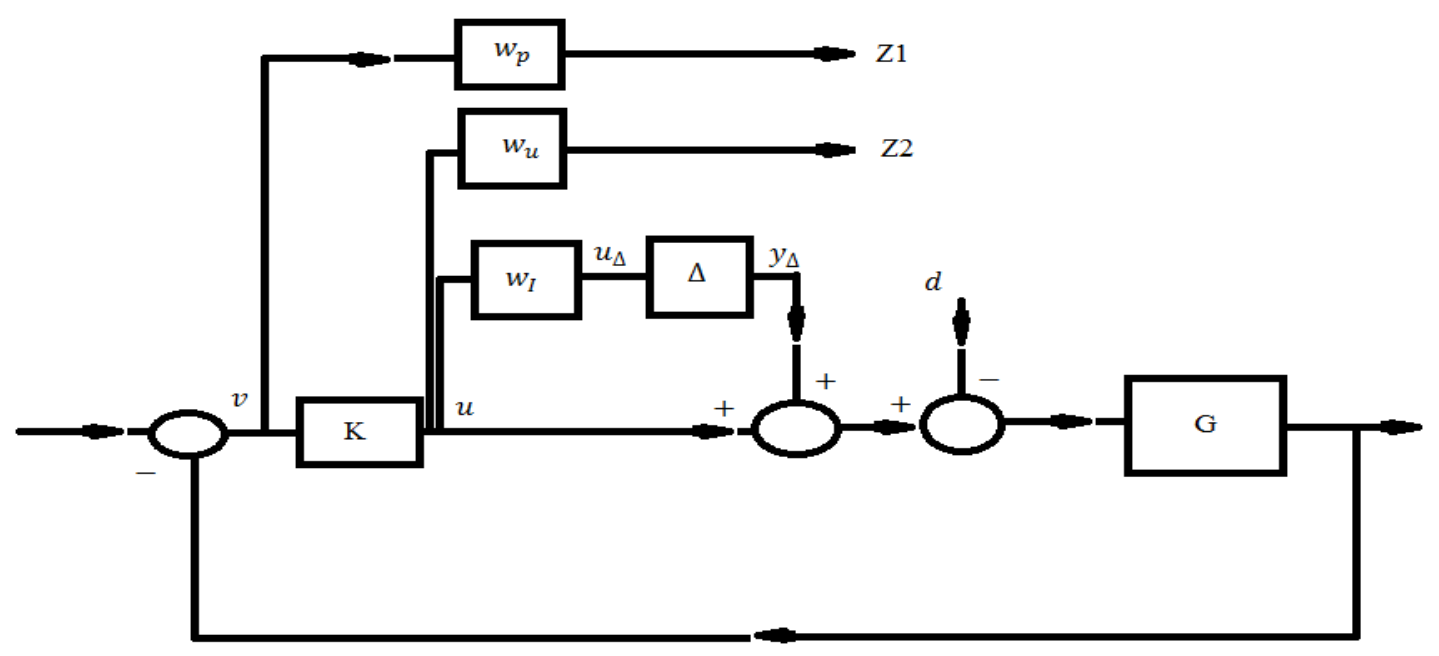

Figure 39. Block diagram of robust control model with multiplicative uncertainty.

\subsubsection{Robust Control Design}

The nominal model was chosen for the control design. The safe level SpO2 is $92 \%$ for a neonatal infant. The controller was designed to reject disturbances that tend to drive the $\mathrm{SpO} 2$ from the nominal set point conditions. The desired bandwidth frequency, $\omega_{b}, 1$ radians/second obtained by finding the bandwidth frequency of the nominal model. A performance weight, $w_{P}(s)$, is multiplied by the error signal that corresponds to a maximum allowed $1 \%$ error at frequencies below the $w_{b}$ and $50 \%$ error at high frequencies. The performance weight, $w_{P}(s)$, is defined as

$$
w_{P}(s)=\frac{\frac{1}{M} s+\omega_{b}}{s+A \omega_{b}}
$$


where $M$ is high frequency, $A$ is the low frequency error, and $\omega_{b}$ is band width for $\left|\frac{1}{w_{p}(j w)}\right|$. The $|S(j w)|$ is the magnitude of error the system and $\left|\frac{1}{w_{p}(j w)}\right|$ be upper bound on $\mathrm{S}$ or largest acceptable error is

$$
|S(j w)|<\left|\frac{1}{w_{p}(j w)}\right| \quad \forall \omega
$$

and for condition above we can get parameters of $w_{P}(s)$ as

$$
w_{P}(s)=\frac{\frac{1}{2} s+\omega_{b}}{s+0.1 \omega_{b}}
$$

Figure 40 shows the Bode diagram of the performance weight, and $w_{u}$, is added onto the control signal to limit its maximum value.

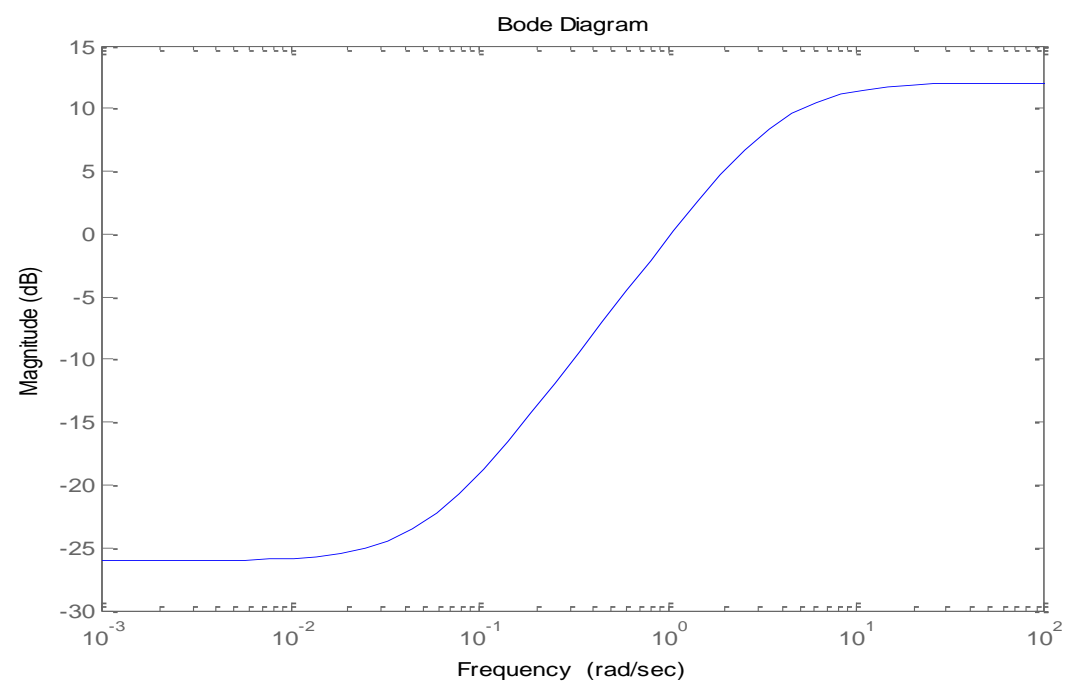

Figure 40. Bode diagram of the $w_{P}$ performance weight 
The block diagram in Figure 24 was transformed to form a diagram of the form in Fig 26. The generalized plant matrix, $\mathbf{P}$, the $\mathrm{P}$ matrix for this system is

$$
\begin{gathered}
{\left[\begin{array}{c}
y_{\Delta} \\
z_{1} \\
z_{2} \\
v
\end{array}\right]=\left[\begin{array}{ccc}
0 & 0 & w_{I} \\
-G w_{P} & w_{P} & -G w_{P} \\
0 & 0 & w_{u} \\
-G & 1 & -G
\end{array}\right]\left[\begin{array}{l}
u_{\Delta} \\
d \\
u
\end{array}\right]} \\
P_{11}=\left[\begin{array}{cc}
0 & 0 \\
-G w_{P} & w_{P} \\
0 & 0
\end{array}\right] \\
P_{12}\left[\begin{array}{c}
w_{I} \\
-G w_{P} \\
w_{u}
\end{array}\right] \\
P_{21}=\left[\begin{array}{ll}
-G & 1
\end{array}\right] \\
P_{22}=\left[\begin{array}{l}
-G
\end{array}\right]
\end{gathered}
$$

where $G_{P}$ is the nominal system gain, $G$ is the nominal plant transfer function, the output $z_{1}$ is connected to the weighted error signal, and output $z_{2}$ is connected to the weighted control signal. The block diagram of the P matrix appears in Figure 41. 


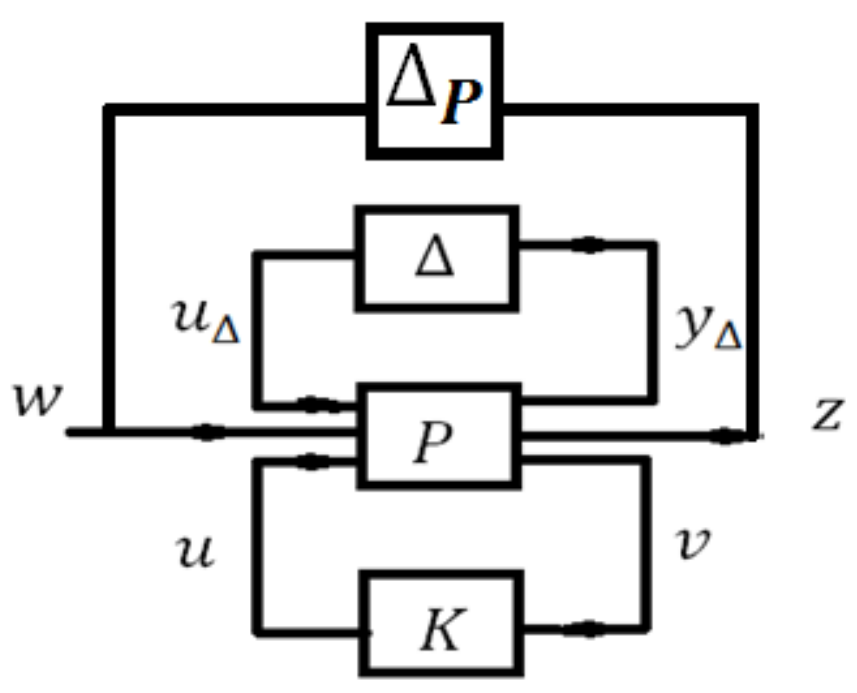

Figure 41. Block diagram of the $P$ matrix structure.

The $\mu$ - synthesis controller optimization is needed since a structure $\hat{\Delta}$ matrix is considered where when the performance and uncertainty are included in the controller optimization. The $\hat{\Delta}$ matrix can be defined as

$$
\hat{\Delta}=\left[\begin{array}{cc}
\Delta & 0 \\
0 & \Delta_{P}
\end{array}\right]
$$

where $\Delta_{P}$ is the performance uncertainty and $\Delta$ is the model uncertainty. 
There is one input $y_{\Delta}$, and one output $u_{\Delta}$ for matrix $\Delta$. There are two inputs $z_{1}$ and $z_{2}$ and one output, $d$ for matrix $\Delta_{P}$. The configuration of $N-\Delta$ can been seen in Figure 42 and it is combined of $\mathrm{P}$ matrix and controller and using the lower fractional transformation (LFT) as

$$
N=P_{11}+P_{12} K\left(I-P_{22} K\right)^{-1} P_{21}
$$

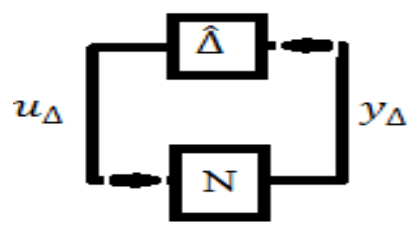

Figure 42. Block diagram of the $N-\Delta$ configuration.

In Matlab, dksyn.m was used to conduct the $\mu-$ synthesis design. The controller has one input, $v$, and one output, $u$. The resulting controller transfer function is

$$
k=\frac{633.2 s+1146}{s^{2}+490.7 s+24.53}
$$

The conditions were checked for robust stability and performance and nominal stability and performance, so we took the matrices $\mathrm{N}$ and $\mathrm{P}$. For the nominal stability, the closed-loop system must be in the left - half plane, so the system is nominally stable. Nominal performance checks to see if the controller performs according to the performance criteria under nominal conditions. The test for nominal performance is given as, 


$$
N P=\left|W_{P(j w)}(1+K G)^{-1}\right|_{\infty}
$$

To check the nominal performance, the inequality must hold for frequencies [32].

$$
\left\|N_{22}\right\|_{\infty} \leq 1
$$

The system is nominally stable if only if the values of $N_{22}$ less than one and can be seen the nominal performance by plotting the H-infinty norm as shown in Figure 43.

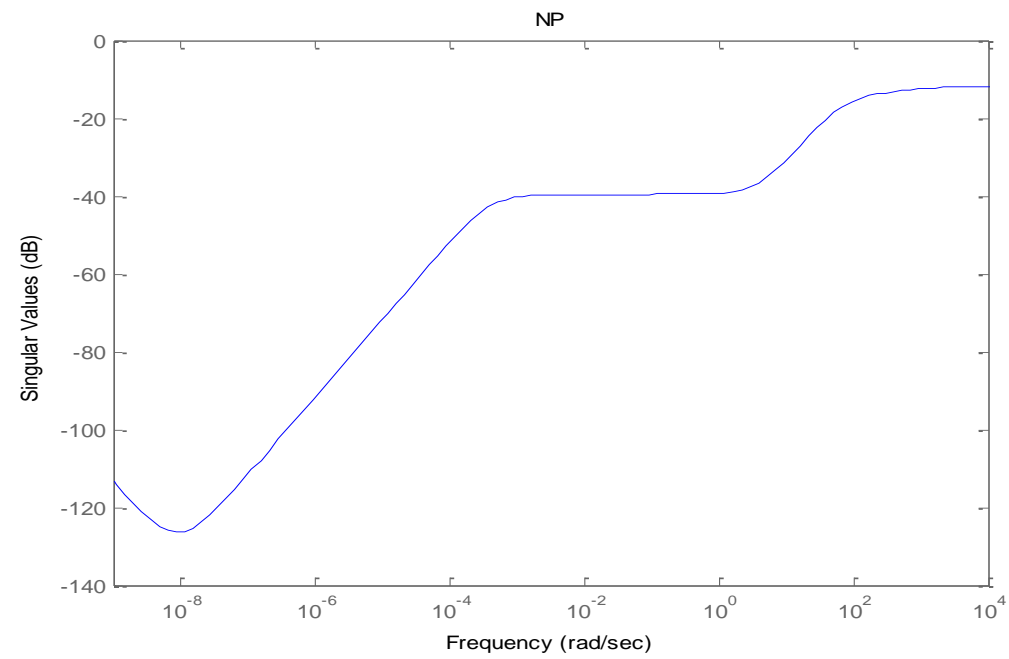

Figure 43. The H-infinity norm of $N_{22}$ is less than one for all frequencies. 
For the robust stability, $N_{11}$ is less or equal one for all frequencies and that the system is robust stable [31]. The equation for robust stability is

$$
R S=\left|W_{i(j w)} K G(1+K G)^{-1}\right|_{\infty}
$$

The inequality

$$
\left\|N_{11}\right\|_{\infty} \leq 1
$$

To achieve robust stability the maximum value from Eq (4.12) must be less than one.

The h-infinity norm of $N_{11}$ can bee seen in Figure (44).

Robust performance checks to see if the controller performs according to the performance criteria over a range of input. Robust performance can be checked using Eq (4.13).

$$
\begin{gathered}
R P=\left|W_{P(j w)}(1+K G)^{-1}\right|_{\infty}+\left|W_{i(j w)} K G(1+K G)^{-1}\right|_{\infty} \\
\mu(N, \hat{\Delta})<1
\end{gathered}
$$

The recursive algorithms and the iterative algorithms can estimate the parameters of linear regressive models from observation data [34-36]. 
The $\mu$-synthesis controller is found to have robust performance which can bee seen in Figure (45). We can see that the amplitudes of max singular value and $\mu$-synthesis are less than one and that means that the robust controller is found to be able to achieve a desirable performance given the level of uncetainty in the system that was modeled.

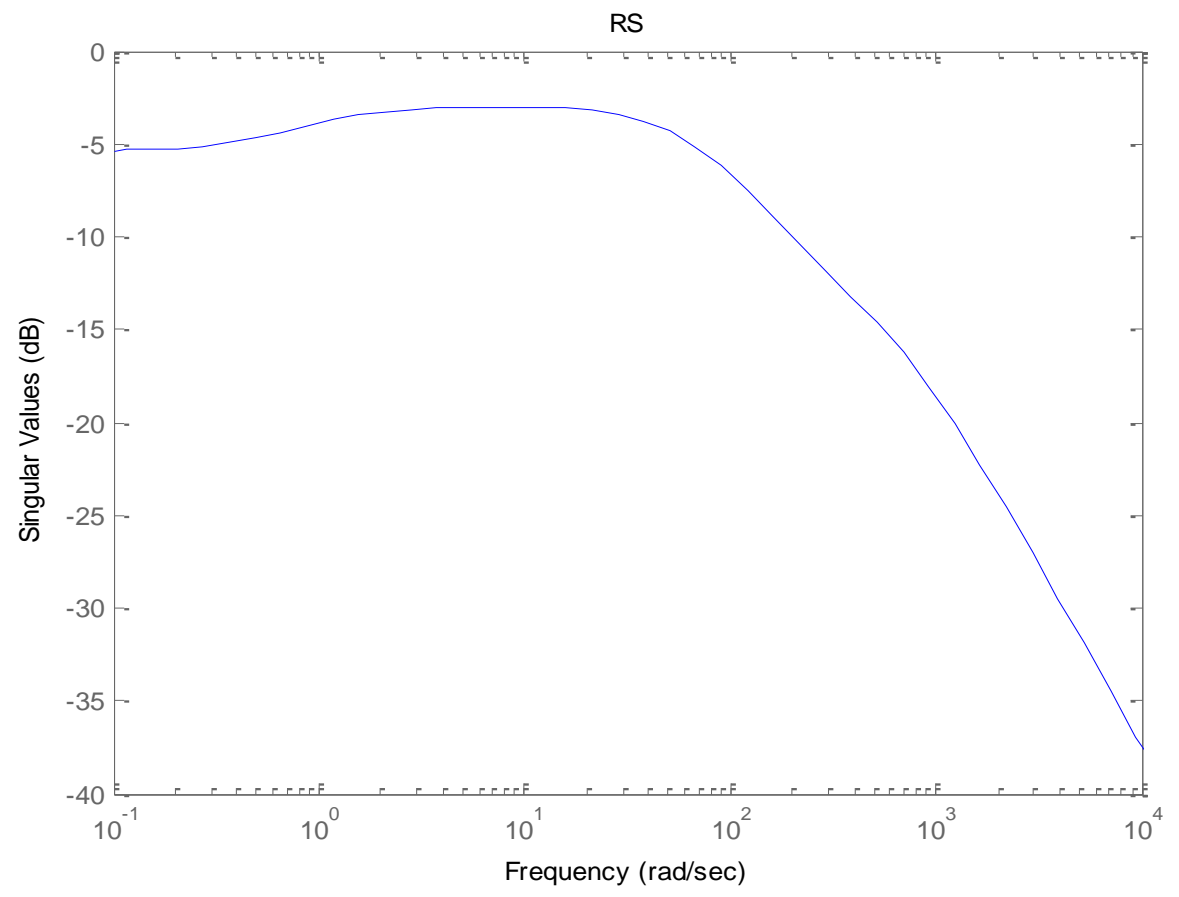

Figure 44. The H-infinity norm of $N_{11}$ is less than one for all frequencies for the $\mu$-synthesis controller. 


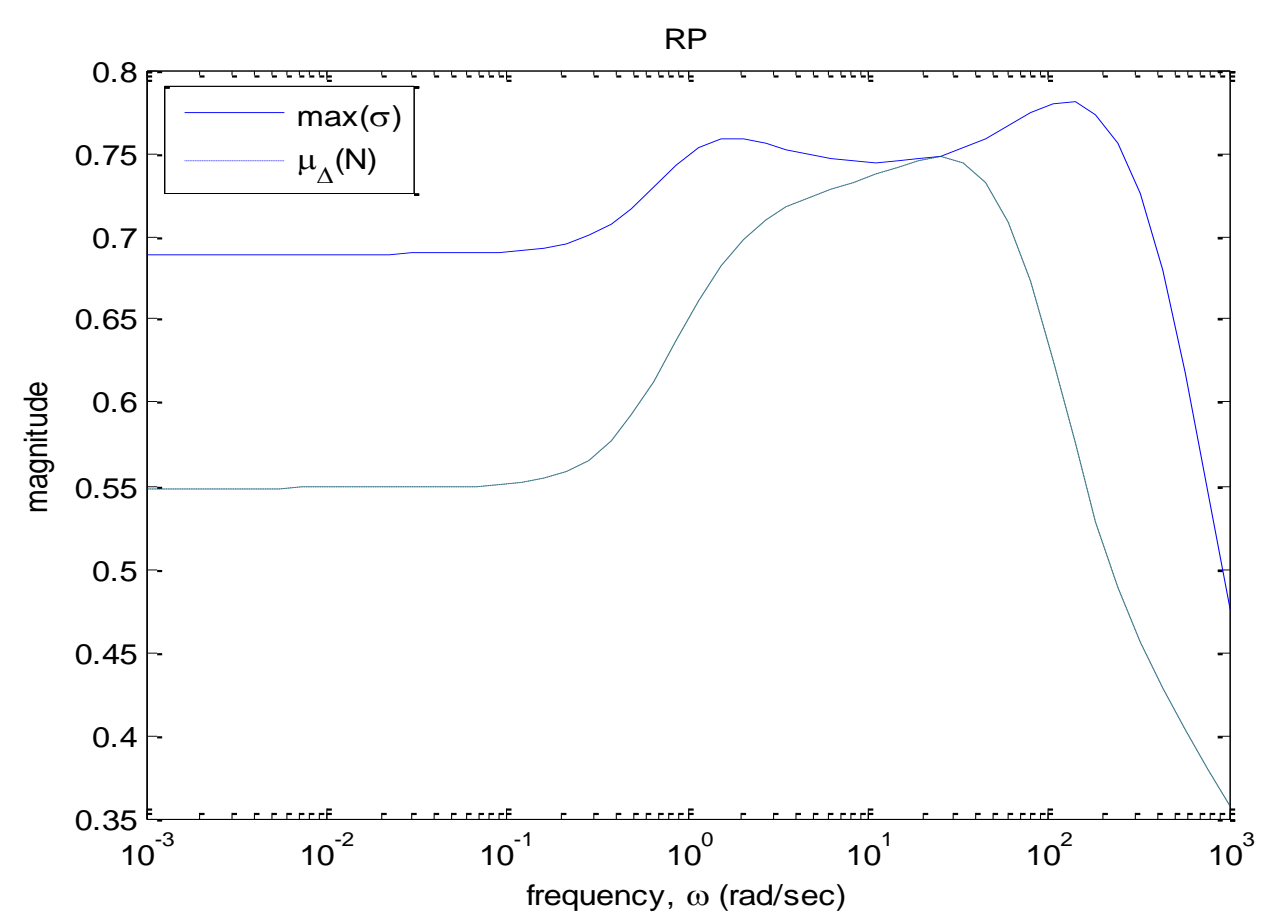

Figure 45. The maximum singular value and structured singular value of the $\mathbf{N}$ matrix is less than one for all frequencies.

\subsection{Robust Control with MPC Controller.}

\subsubsection{Recursive Least Square (RLS)}

For robust performance, the structured singular value of $\mathrm{N}$ must be below one for the entire frequency range and can be defined by the inequality in some matrix equations, and the parameter estimation algorithm can be implemented in a recursive form. Therefore, it is necessary to compute the criterion function in a recursive form since the criterion function values can measure the parameter 
estimation accuracy [37]. To estimate the model control system that in model predictive section, we must find the coefficients of the polynomial of estimate system. This is usually accomplished by assuming a discrete time form for the control system model and then using a recursive estimation algorithm to obtain estimates of the parameters of the model. To determine the coefficient of the model parameters using the recursive least square, a scheme of new input/output data becomes available at each sample interval. The model based on past information (summarized in $\theta^{\prime}(t-1)$ as a vector of unknown) is used to obtain an estimate $y(t)$ to generate an error $\varepsilon(t)$. This in turn generates an update to the model which corrects $\theta^{\prime}(t-1)$ to the new value $\theta^{\prime}(t)$. This recursive "predictor corrector" form allows significant saving in computation, requiring the storage of all previous data. It is both efficient and elegant to merely store the "old" estimate calculated at timet, denoted $\mathrm{b} \theta^{\prime}(t)$, and to obtain the "new" estimates $\theta^{\prime}(t+1)$ by an updating step involving the new observation only. Recursive Least Square (RLS) is used as on - line identification [38 - 39].

The algorithm below was used to calculate the recursive least square.

(i) From $x(t+1)$ using the new data.

(ii) From $\varepsilon(t+1)$ using $X^{T}(t+1)$.

$$
\varepsilon(t+1)=y(t+1)-X^{T}(t+1) \quad \theta^{\prime}(t)
$$


(iii) From using

$$
p(t+1)=p(t)\left[I_{m}+\frac{x(t+1) X^{T}(t+1) p(t+1)}{1+X^{T}(t+1) p(t) x(t+1)}\right]
$$

(iv) Update $\theta^{\prime}(t)$

$$
\theta^{\prime}(t+1)=\theta^{\prime}(t)+p(t+1) x(t+1) \varepsilon(t+1)
$$

(v) Wait for the next time step to elapse and loop back to step (i).

Now, we used RLS in the algorithm above to get an estimate of the control modeling system to get the discrete differential equation $y(n)$ and after that to convert the discrete equation to z-domain and after that convert to s- domain by using bilinear equation as shown in Figure 46.

The discrete differential equation for control in MPC after RLS algorithm is

$$
\begin{gathered}
u(n)=0.01529 u(n-1)+0.0075 u(n-2)+0.7226 u(n-3) \\
+0.5515 e(n-1)+0.5515 e(n-2)
\end{gathered}
$$

The Laplace transforms function for control in MPC after converting z- transform to $\mathrm{s}$-domain is

$$
\frac{u(s)}{e(s)}=\frac{0.1234 s^{2}+0.8769 s+1.879}{s^{3}+0.3257 s^{2}+4.3313 s+0.08534}
$$




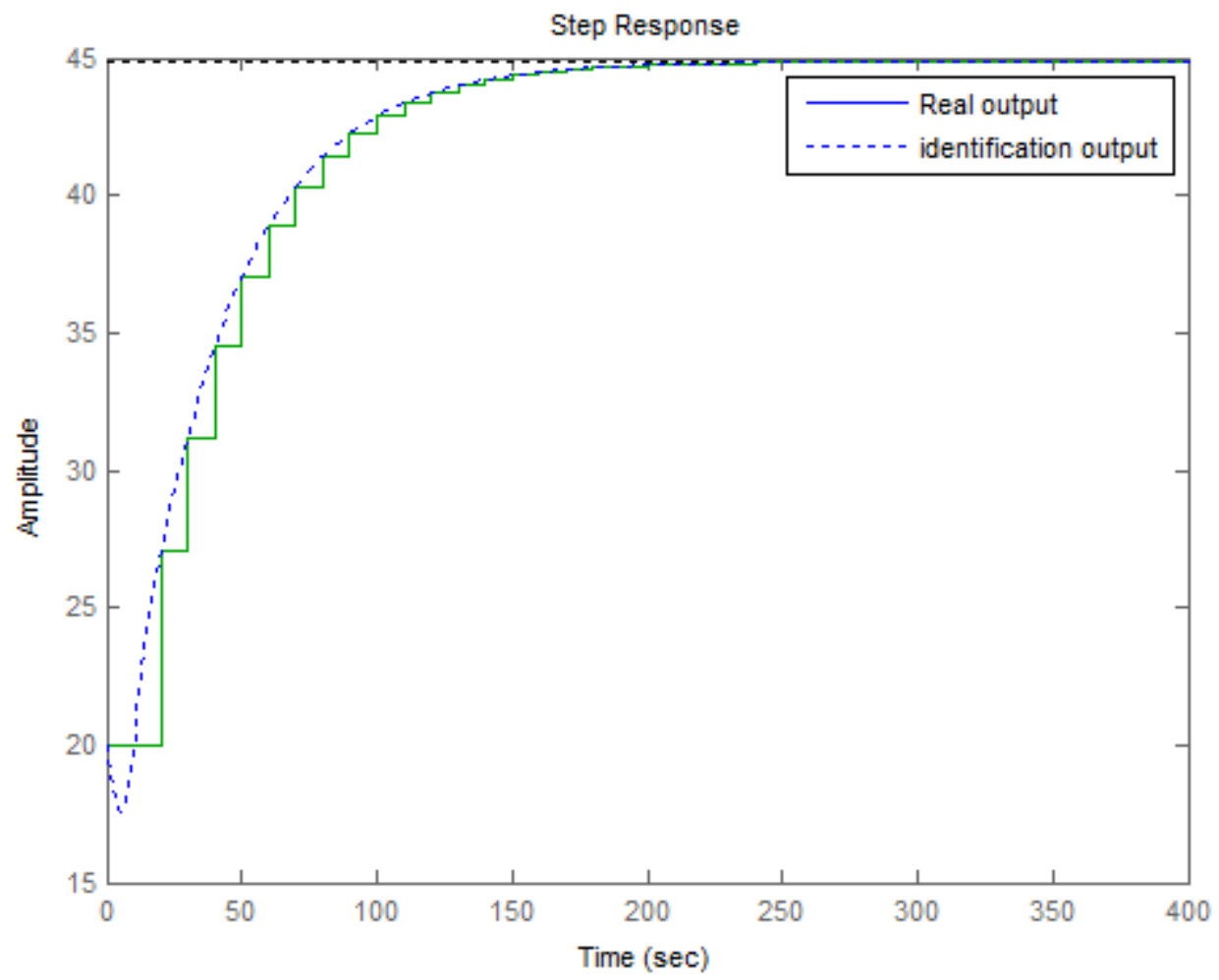

Figure 46. The real control output with the identification by RLS.

\subsubsection{Robustness with Model Predictive Control.}

The nominal model was chosen for control design. The safe level $\mathrm{SpO} 2$ is $92 \%$ for a neonatal infant. The controller was designed to reject disturbances that tend to drive the $\mathrm{SpO} 2$ from the nominal set point conditions. The desired bandwidth frequency, $\omega_{b}, 1$ radians/second obtained by finding the bandwidth frequency of the nominal model. A performance weight, $w_{P}(s)$, is multiplied by the error signal that corresponds to a maximum allowed $1 \%$ error at frequencies 
below the $w_{b}$ and $50 \%$ error at high frequencies. The performance weight, $w_{P}(s)$, is defined as

$$
w_{P}(s)=\frac{\frac{1}{M} s+\omega_{b}}{s+A \omega_{b}}
$$

where $M$ is high frequency, $A$ is the low frequency error, and $\omega_{b}$ is band width for $\left|\frac{1}{w_{p}(j w)}\right|$. The $|S(j w)|$ is the magnitude of error the system and $\left|\frac{1}{w_{p}(j w)}\right|$ be upper bound on $\mathrm{S}$ or largest acceptable error is

$$
|S(j w)|<\left|\frac{1}{w_{p}(j w)}\right| \quad \forall \omega
$$

and for condition above we can get parameters of $w_{P}(s)$ as

$$
W_{P}(s)=\frac{\frac{1}{6} s+0.05}{s+0.5 * 0.05}
$$

Figure 47 shows the Bode diagram of the performance weight and $w_{u}$, is added onto the control signal to limit its maximum value. 


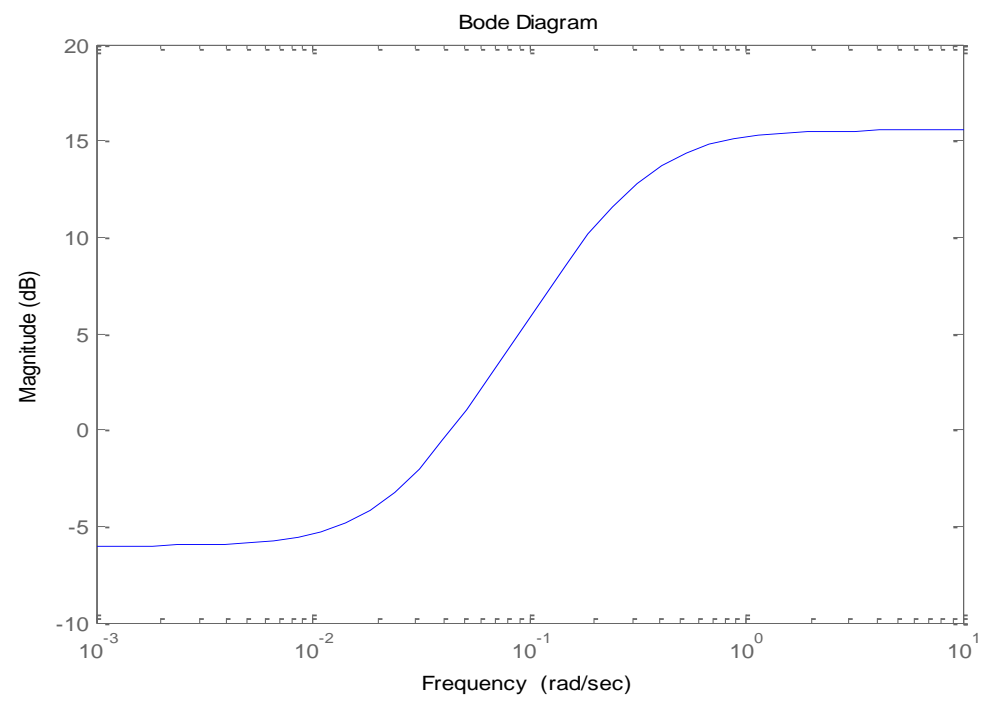

Figure 47. Bode diagram of the $w_{P}$ performance weight.

The conditions were checked for robust stability and performance and nominal stability and performance, so we took the matrices $\mathrm{N}$ and $\mathrm{P}$. For the nominal stability, the closed-loop system must be in the left - half plane, so the system is nominally stable. We performed nominal performance checks to see if the controller performs according to the performance criteria under nominal conditions. The test for nominal performance is given as

$$
N P=\left|W_{P(j w)}(1+K G)^{-1}\right|_{\infty}
$$

To check the nominal performance, the inequality must hold for frequencies [32]. 


$$
\left\|N_{22}\right\|_{\infty} \leq 1
$$

The system is nominally stable if only if the values of $N_{22}$ are less than one and can be seen the nominal performance by plotting the H-infinty norm in Figure 48.

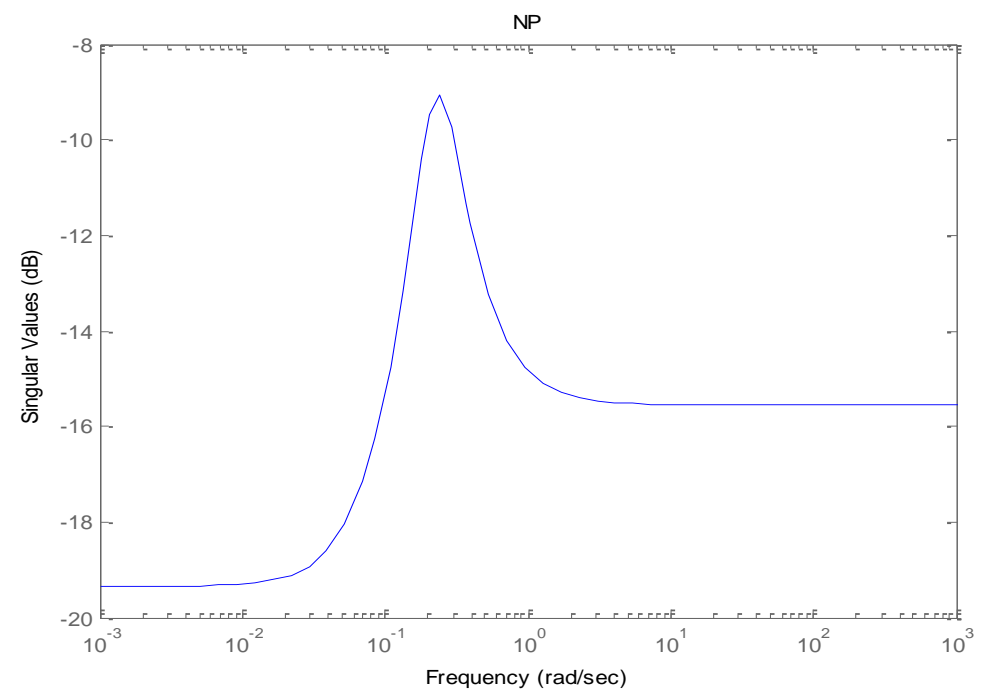

Figure 48. The H-infinity norm of $N_{22}$ is less than one for all frequencies.

For the robust stability, $N_{11}$ is less or equal one for all frequencies and the system is robust stable [31]. The equation for robust stability is

$$
R S=\left|W_{i(j w)} K G(1+K G)^{-1}\right|_{\infty}
$$

The inequality 


$$
\left\|N_{11}\right\|_{\infty} \leq 1
$$

To achieve robust stability the maximum value from Eq (4.12) must be less than one.

The h-infinity norm of $N_{11}$ can bee seen in Figure (49).

Robust performance checks to see if the controller performs according to the performance criteria over a range of input. Robust performance can be checked using Eq (4.13).

$$
R P=\left|W_{P(j w)}(1+K G)^{-1}\right|_{\infty}+\left|W_{i(j w)} K G(1+K G)^{-1}\right|_{\infty}
$$

For robust performance, the structured singular value of $\mathrm{N}$ must be below one for the entire frequency range and can be defined by the inequality

$$
\mu(N, \hat{\Delta})<1
$$

The $\mu$-synthesis controller is found to have robust performance which can bee seen in Figure 50. We can see that the amplitudes of max singular value and $\mu$-synthesis are less than one and that means that the robust controller is 
found to be able to achieve a desirable performance given the level of uncetainty in the system that was modeled.

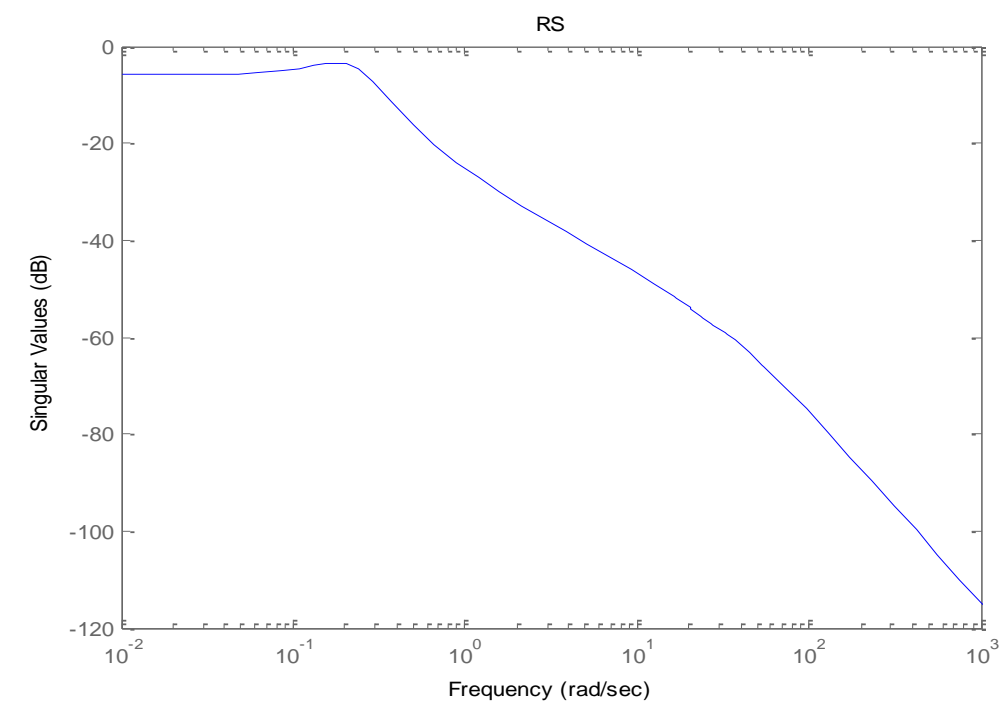

Figure 49. The H-infinity norm of $N_{11}$ is less than one for all frequencies for the $\mu$-synthesis controller.

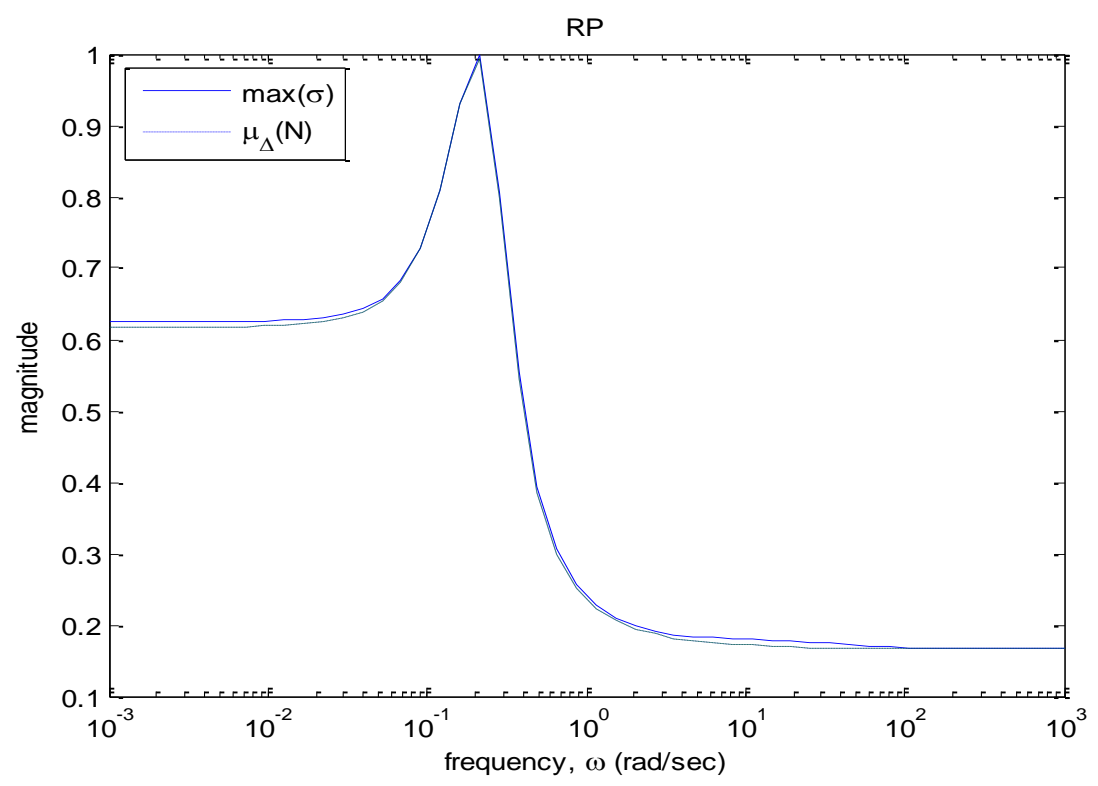

Figure 50. The maximum singular value and structured singular value of the $\mathrm{N}$ matrix is less than one for all frequencies. 


\section{Chapter 5. Illustration of Prototype}

\subsection{Overview of Device}

The most problems for nurses are how takes care in the neonatal intensive care unit to adjust $\mathrm{FiO}_{2}$ setting. The aim of this prototype is to maintain the level of care to neonate to help the nurses to reduce manual intervention and time that is needed to react to the alarms. The block diagram of device can be seen in Figure 51.

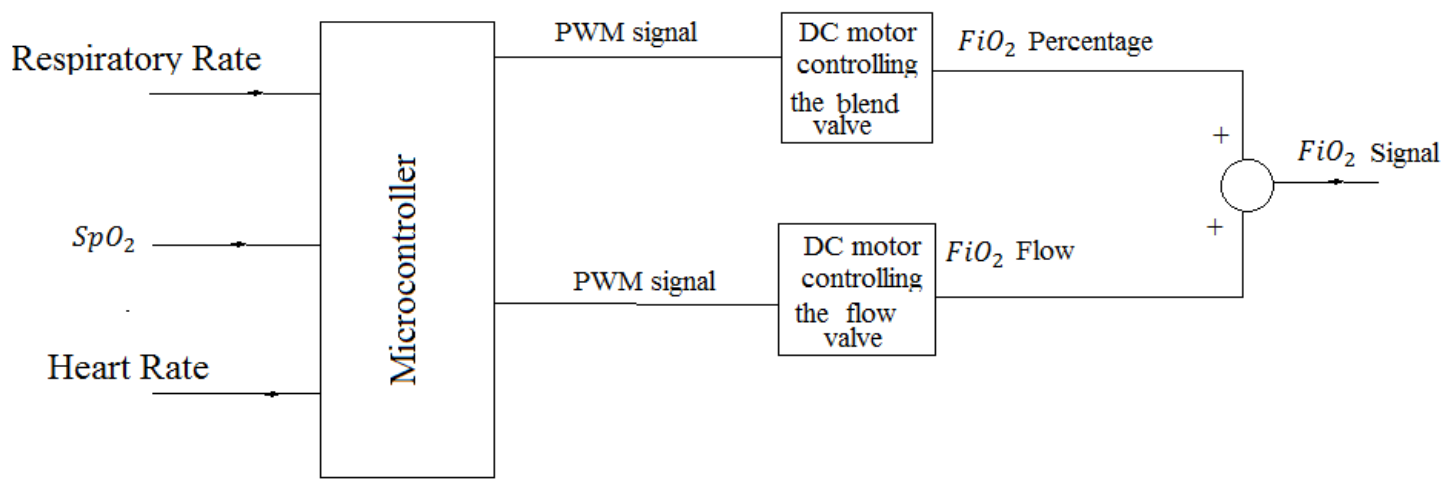

Figure 51. Block Diagram of the device and connections.

The main branch of the whole devices consists of how to design it to turn the blend valve automatically. The DC motor has a built in encoder to measure the angular position of the motor shaft as shown in Figure 52.a. The angular position of the motor shaft can be measured by a DC motor as shown in Figure 52. b. The 
motor is controlled by a National Instruments sbRIO microcontroller. There are two motors and four port serial adapter for microcontroller.

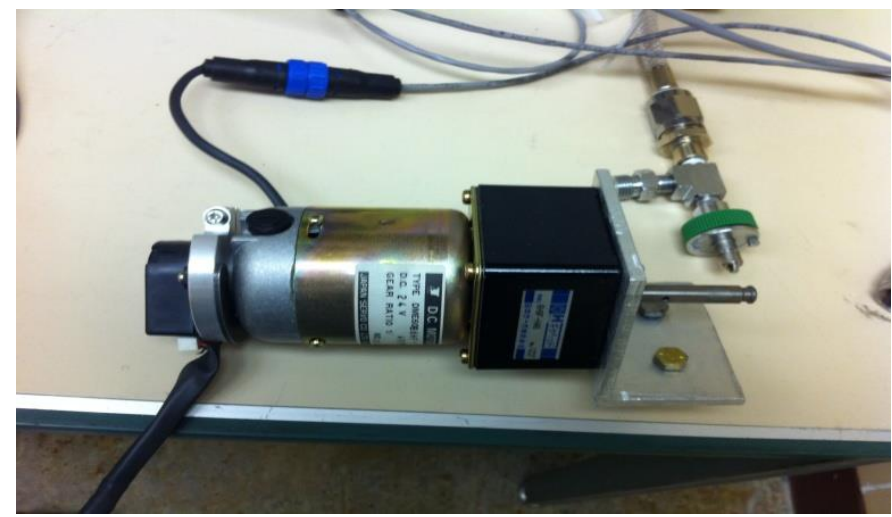

Figure 52. a) DC motor with encoder. 


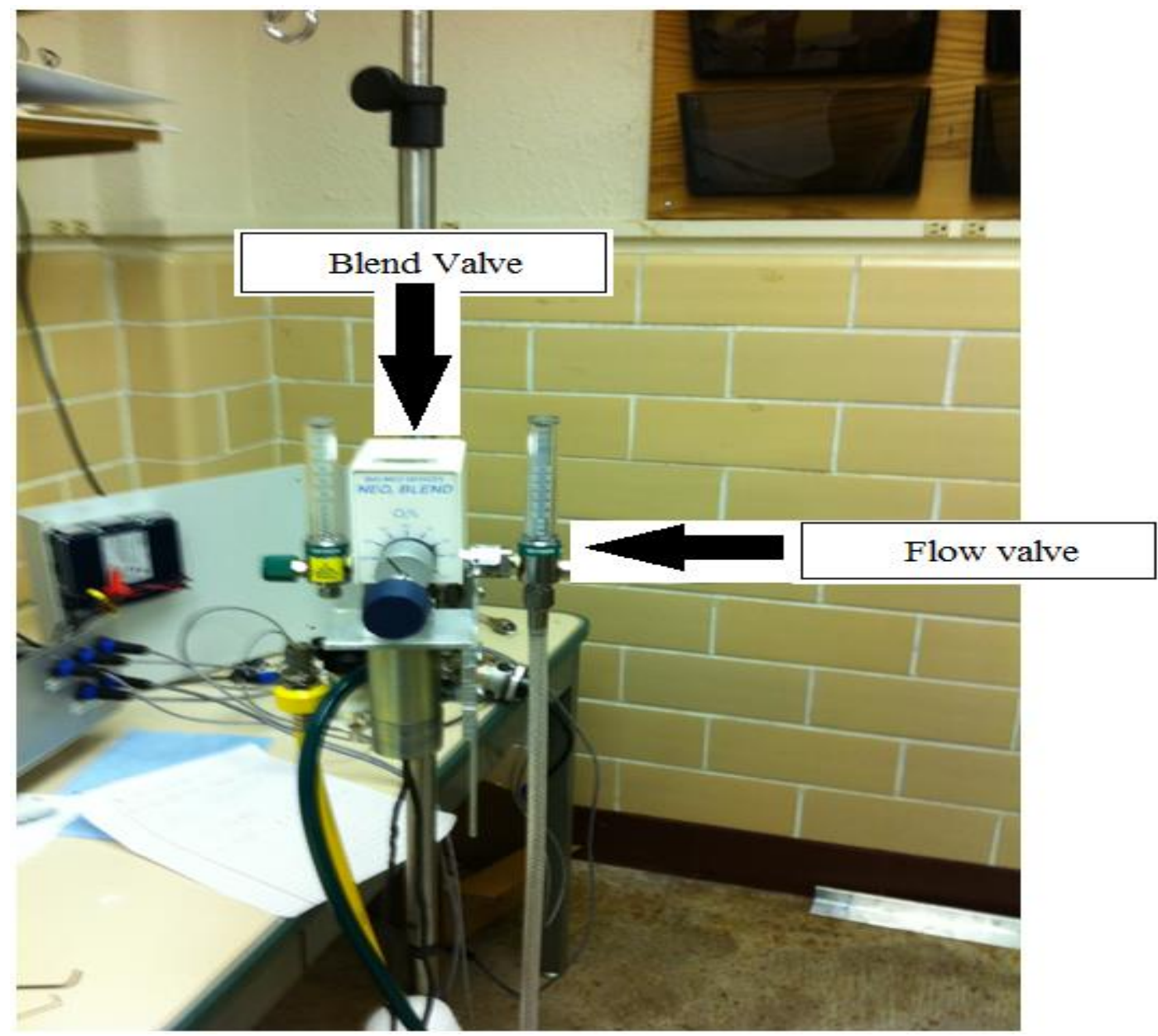

(b)

Figure 52. b. Knob tuning device connected to the blend valve knob.

The microcontroller will sample the patient's vital signs from the port of Spacelab patient monitor, and these signals will be processed by software programming that is loaded onto the microcontroller via a laptop that is connected by link cable. The microcontroller can adjust the value of $\mathrm{FiO}_{2}$ percentage based on patient vital signs and we can see the microcontroller in Figure 53. 


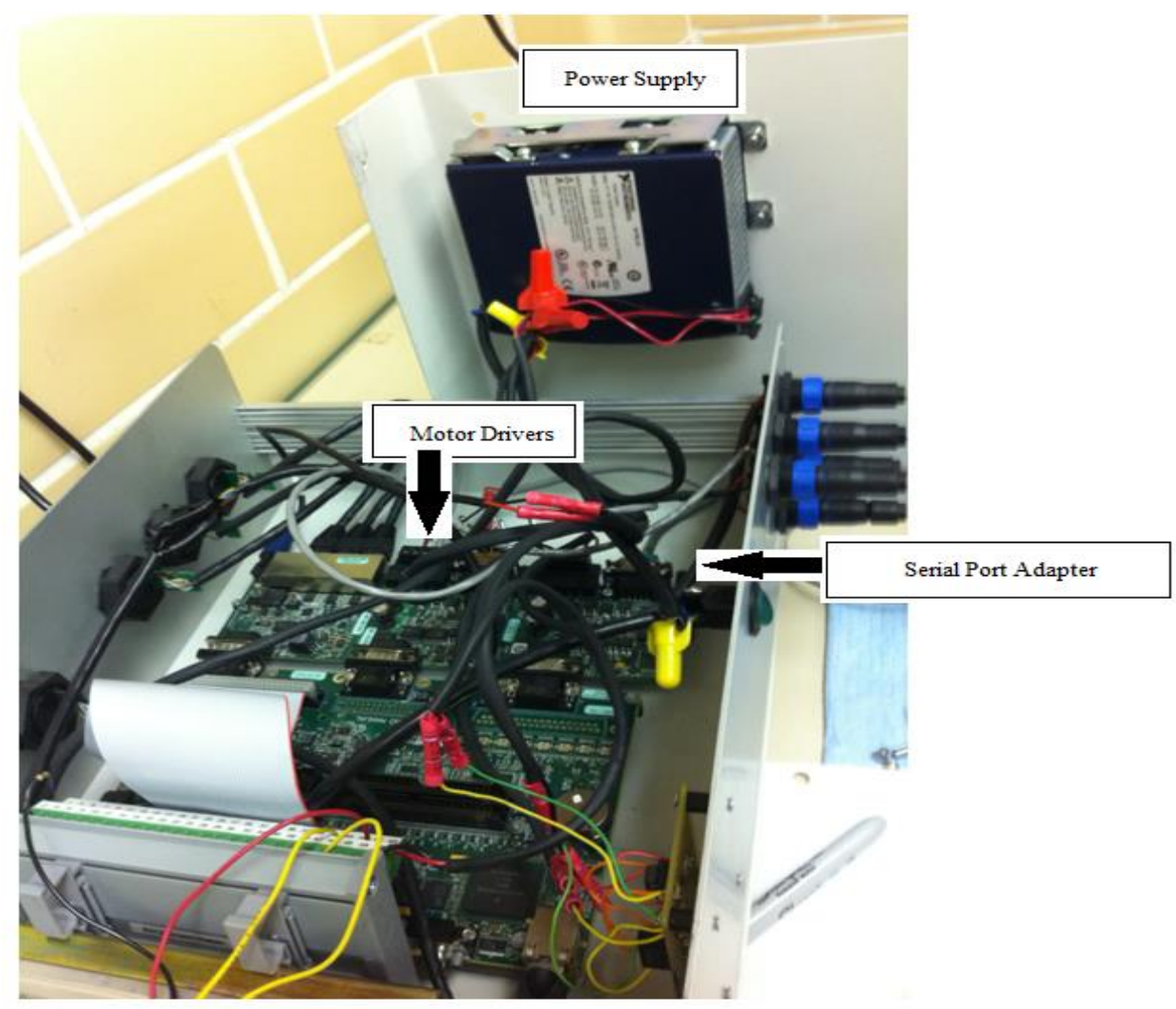

Figure 53. The National Instruments sbRIO microcontroller and power supply. 


\section{Chapter 6: Conclusion and Signification}

\subsection{Conclusion of Data}

A model for neonatal infants developed by $\mathrm{Yu}$ [12] was selected because there is one input that is $\mathrm{FiO}_{2}$ and other modes depend on lumped parameter and non-invasive measurements. The main problem is how we can introduce $\mathrm{FiO}_{2}$ to produce $\mathrm{SpO}_{2}$ between $85 \%$ to $93 \%$ to keep the infants alive without suffering from hypoxemia. In this dissertation, we designed PI, PID, MPC, Robust PID, and Robust MPC controllers to compare between them, which is the best model to use. The goal is to reduce the amount and spending time of harmful desaturation for the neonate and to reduce some of the demanding workload of nurses in the neonatal intensive care unit. The most points that are investigated in this dissertation are mathematical modeling, identification, controllers, and prototype instruction.

A nonlinear system model is developed on the system model by $\mathrm{Yu}$ [12]. This system model is chosen because it has one input and one output, and does not require invasive measurements to simulate, the input is $\mathrm{FiO}_{2}$ for a control input and output $\mathrm{SpO}_{2}$ for system model comparison. The linear system model simulates how the premature infant recovers after a desaturation event. By taking a first - order Taylor series to linearization and after that we got a linear equation with one input and one output. 
In the PI digital controller, we achieved a good response for output without zero steady state, and the minimum settling time was $180 \mathrm{sec}$. That needs more estimated values for $K_{p}$ and $K_{I}$ after converting the control law from the continuous domain to the discrete domain by bilinear method. The PI controller has large overshoots and large steady state error when using large values of $K_{I}$ but when decreasing the values of $K_{I}$ got good response with low overshoot and zero steady.

In the PID controller, we got a good response for output without zero steady state, and the minimum settling time at $170 \mathrm{sec}$. that needs more estimated values for $K_{p}, K_{I}$ and $K_{d}$ after converting the control law from the continuous domain to the discrete domain by bilinear method. The PID controller has large overshoots and large steady state error when using large values of $K_{I}$ and small values of $K_{p}$ but when decreasing the values of $K_{I}$ and increasing values of $K_{p}$ got good response with low overshoot and zero steady.

The Model Predictive controller was very good in response for $\mathrm{SpO}_{2}$ with the input value for control input $21 \%$. MPC needs to be produced first to find the prediction model, then it must be complete enough to fully capture the process. In this work, we used the step response produced by DMC to predict the future response of plant. DMC is a form of control algorithm in which the current control action is obtained by solving a finite horizon open-loop optimal control. This process is repeatedly done for each sampling point. The optimization yields an optimal control sequence, and the first control in this sequence is applied to the 
plant. The parameters of control horizon and prediction horizon should be kept short to reduce the computational effort. We took some parameters for prediction control and the control horizon to see which values are useful for designing. In general, we concluded that the value of the control horizon should be small to keep the computational effort manageable, and the value of prediction horizon should large as possible to completely capture the consequences of the control action. On the other hand, the weight matrix was very important for the output to be close to its reference trajectory that we wanted for $p O_{2}$. The larger the weight is for a particular output, the larger is the contribution of its sum of squared deviations to the objective.

Finally, the manipulated variable that makes the output follow a given trajectory moves, and the relative magnitude of weight matrix determined the trade - off between following the trajectory closely and reducing the action of the manipulated variable. In the result from the design of the MPC, we achieved $\mathrm{SpO}_{2}$ to be a minimum settling time of $105 \mathrm{sec}$ and zero steady state error.

A robust control system based on the PID controller was introduced to the plant using the range of parameters found from Bradley Krone's thesis [30]. For the robust control system, a nominal model is chosen and perturbations due to system parameters from this nominal model are used to create a multiplicative error model. A $\mu$ - synthesis controller optimization is used to find a controller such that the $H_{\infty}$ norm between the inputs and outputs must be less than one. . We showed that the result of controller can guarantee stability and performance for whole range of model parameters. Robust model predictive controller was 
analyzed, we did get the robust stability, nominal performance and robust performance. The robust controller is found to have a robust stability and performance, but with a low bandwidth frequency due to a conservative control design required to achieve robust stability with an extremely high level of model error.

In the robust controller with MPC, we estimated modeling for controller in MPC by RLS and got an accurate response for $\mathrm{FiO}_{2}$ by supplying error as input to get modeling. By converting function of the controller from discrete to continuous and put it as a function in $\mathrm{s}-$ domain to use it in robust control. The result we got was very accuracy with an error model created a $\mu$ - synthesis controller optimization routine. The main goal of the robust controller was analyzed for performance and stability. It was shown to be more nominally stable and have nominal performance and robust stability and performance. We showed that the result of controller can guarantee stability and performance for a whole range of model parameters.

We designed a robust control with Model Predictive Control to get a high accuracy response to be more nominally stable and to have nominal performance and robust stability and performance. We showed that the result of this controller can guarantee stability and performance for a whole range of model parameters. Therefore, the Robust MPC is superior to other controllers because it is more accurate to prove stability and performance for whole range of model parameters. 
The robust with PID and robust with MPC control systems are compared to a static PI, PID, and MPC controllers. The PI and PID digital controller are designed by using bilinear method and by trial and error. The controllers are tested using a simulation with varying parameters. The PI controller has large overshoots and large steady state error when using large values of $K_{I}$ but we got good response with low overshoot and zero steady stare with varying low values of $K_{I}$ but it is less accurate than the robust MPC controller. The PID controller has low overshoot and zero steady state error with decreasing the values of $K_{I}$ and increasing values of $K_{p}$ but we got that with many attempts for trial and error. In MPC is very accurate with no overshoot and zero steady state, and faster than using PI and PID. The robust with PID is found to have robust stability and performance but the robust with MPC is more accurate guarantee stability and performance for whole range of model parameters.

Future research on controller for arterial saturation in neonatal in infants should investigate the use of the Robust MPC in more neonates because it has range of uncertainty and more robust stability and performance to alleviate a load work for nurses. 


\section{Appendices}

\section{Appendix A: Nomenclature}

RDS Respiratory distress syndrome

ROP Retinopathy of prematurity

$\mathrm{S}_{p} \mathrm{O}_{2} \quad$ Arterial oxygen saturation

$\mathrm{F}_{i} \mathrm{O}_{2} \quad$ Fraction of inspired oxygen

$V_{A} \quad$ Ventilated part of the lung is perfused with blood

$\dot{V}_{I} \quad$ Total respiratory rate

$x_{d} \quad$ Dead space ratio

$\dot{V}_{A} \quad$ Respiratory rate of the $V_{A}$ section of the lung model

$Q_{p} \quad$ Pulmonary blood flow

$Q \quad$ Total blood flow to respiratory system

$P_{A} \quad$ The alveolar oxygen partial pressure in the lung

$C_{c} \quad$ The oxygen content of pulmonary end - capillary blood in vol\%

$C_{v} \quad$ The oxygen content of mixed venous blood in vol\% 
PT The patient's temperature

$P_{v} \quad$ The partial pressure of oxygen in the venous blood

$\Delta P_{A} \quad$ Linearized alveolar oxygen partial pressure in the lung

$\Delta P_{I} \quad$ Linearized partial pressure of oxygen in inspired air

$\beta_{a} \quad$ Apparent solubility of oxygen in arterial blood

$\beta_{c} \quad$ The apparent solubility of oxygen in whole blood in the alveolar

$y_{s} \quad$ Shunt ratio

$P_{a} \quad$ Partial pressure of oxygen in the artery

$P_{H 2 o} \quad$ Water vapor pressure

$P_{B} \quad$ Barometric pressure

Gp Steady state system gain

$G_{p c} \quad$ Total steady state system gain for linear system

$\tau \quad$ Time constant for the lung model

$s \quad$ Laplace variable

$P_{a} \quad$ Partial pressure of oxygen in the artery

$G_{c} \quad$ Oxygen dissociation curve

$K_{a} \quad$ Alveolar - arterial oxygen difference 
$\Delta P_{I} \quad$ Linearized partial pressure of oxygen in inspired air

$K_{p} \quad$ Proportional gain

$K_{d} \quad$ Derivative gain

$K_{i} \quad$ Integral gain

$u(s) \quad$ The output of controller

Z Z - Transform variable

T Discrete sampling time

$\hat{y}(t+k)$ Predicted output at future instants

$g_{i} \quad$ Sampled output values for the step input

$\Delta u(t) \quad$ Variation of step input

$h_{i} \quad$ Sampled output

G System's dynamic matrix

$p \quad$ Dimensional vector of the system prediction along the horizon

$m \quad$ Dimension vector of control increment

$\Gamma_{l}^{y} \quad$ Weighting matrices to penalize particular components of $y$

$\Gamma_{l}^{u} \quad$ Weighting matrices to penalize particular components of $u$

$y(t+1) \quad$ Output predicted over a horizon $p$ 
$r(k+1) \quad$ Vector of future reference values (set point)

$E_{p}(k+1 \mid k)$ Vector of predicted future errors over the horizon $p$

$K_{M P C} \quad$ Stability of the closed - loop system gain

$W_{I}(s) \quad$ Multiplicative uncertainty transfer function weight

$G_{p}(j \omega) \quad$ Perturbation plant used in error modeling analysis

$G_{n o m}(j \omega) \quad$ Nominal plant used in error modeling analysis

$E_{I}(j \omega) \quad$ Multiplicative uncertainty error

$w_{P}(s) \quad$ Error performance weight

$w_{u}(s) \quad$ Control signal performance weight

$\omega_{b} \quad$ Bandwidth frequency of the error performance weight

$\Delta \quad$ Model uncertainty

$\Delta_{P} \quad$ Performance uncertainty

$K(s) \quad$ Robust control

$P \quad$ Generalized plant matrix

$X(t) \quad$ Output and input matrix

$\varepsilon(t) \quad$ Error between the true outputs with estimation output

$\theta^{\prime}(t) \quad$ Estimation output 
$p(t) \quad$ Auto - correlation matrix 


\section{References}

1. "Neonatal respiratory distress syndrome" at Dorland's Medical Dictionary. 29 th $^{\text {th }}$ Edition 2012.

2. R.J. Rodriguez, R.J. Martin, and A.A. Fanaroff, "Respiratory distress syndrome and its management, "Fanaroff and Martin (eds.) Neonatal-perinatal medicine: Diseases of the fetus and infant; 7th ed.1001-1011. St. Louis: Mosby 2002.

3. L. Sandra, C. Brian, A. Jacinto, Hemandez, I. Mary, "Merenstein \& Gardner's handbook of Neonatal Intensive Care" Seventh edition 2010.

4. T. Keim, R. Amjad, and Roger, F., "Modeling and Control of the Oxygen Saturation in Neonatal Infants," Dynamic System and Control Conference Arlington, VA, pp. 1-2, 2009.

5. A. Lapttok, W. Salhab, J. Allen, S. Saha, and M. Walsh, “ Pulse Oximetry in Very Low Birth Weight Infants: Can Oxygen Saturation by Maintained in the Desired Ranges?," Perinatol, pp. 337-341, 2006

6. Shyang - Yun Pamela K. Shiao and Ching-Nan Ou "Validation of Oxygen Saturation Monitoring in Neonates," American Association of Critical - Care Nurses, 2007

7. L. Roa and I. Ortega-Martinez" Dynamic analysis of the respiratory system," presented at the $19^{\text {th }}$ International IEEE conference, U.S.A.IL, 1997. 
8. M. Revow, S. England, H. O'beirne, and A. Bryan. "A model of the maturation of respiratory control in the newborn infant," in IEEE transaction on Biomedical Engineering, 1989.

9. F. Tehrani "Mathematical analysis and computer simulation of the respiratory system in the newborn infant," in IEEE transaction on Biomedical Engineering, 1993.

10. S. Kanae, K. Muramatsu, Z. Yang and K. Wada, "Modeling of respiration and estimation of pulmonary elastance," presented in $5^{\text {th }}$ Asia control conference, 2004.

11. S. Sands, B. Edwards, V. Kelly, M. Davidson, M. Wilkinson and P. Berger "A model analysis of arterial oxygen desaturation during apnea in preterm infants," in PLOS Computational Biology, 2009

12. C.L. Yu, "An Arterial Oxygen Saturation Controller," Rensselaer Polytechnic Institute, 1986.

13. L. Zhang, R. Cameron "A Real -Time Expert Control Strategy for Blood Gas Management in Neonates Under Ventilation Treatment," Report in Department of Control Engineering, 1988.

14. F. Tehrani and A. Bazar "An Automatic Control for Oxygen Therapy of Newborn Infants," Annual International conference of the IEEE Engineering in Medicine and Biology Society, 1991.

15. M. Taube and M. Blutani "Automatic Control of Neonatal Fractional Inspired Oxygen," Annual International conference of the IEEE Engineering in Medicine and Biology Society, 1991. 
16. C. Yu, W. He, J. So, R. Roy and H. Kaufman, "Improvement In Arterial Oxygen Control Using Multiple Model Adaptive Control Procedures," in Department of Biomedical Engineering Rensselear Polytechnic Institute, NY.

17. P. Morozoff, Evans, R., and Smyth, J., "Automatic Control of Blood Oxygen Saturation in Premature Infants," presented at the Second IEEE Conference on Control Applications, Vancouver, B.C., 1993.

18. T. Keim, and F. Roger, "Control of Arterial Oxygen Saturation In Premature Infants," Dissertation presented to the Faculty of the Graduated school at the University of Missouri, 2011.

19. D. Chumlert, W. Anan, and K. Chitsakul, "Automatic Control for Oxygen Intake via Nasal Cannula," Proceeding of the International MultiConference of Engineers and Computer Scientists, 2010.

20N. T. Claure, R. Gerhardt, G. Evert, C. Musante, and E. Bancalari, "Closed - Loop Controlled Inspired Oxygen Concentration for Mechanically Ventilated Very Low Weight Infants with Frequent Episodes of Hypoxemia," official Journal of the American Academy of Pediatrics, 2001.

21. S. Anand, V. Aswin and S. Kumar, "Simple Tuned Adaptive PI Controller for Conical Tank Process," International Conference on Recent Advancements in Electrical, Electronics and Control Engineering, 2011.

22. R. Youof, S. Omatu and M. Khalid, "Application of Self-Tuning PI Controller to a Temperature Control System," published in IEEE, 1994. 
23. S. Tunyasrirut, J. Ngamwiwit and T. Furuya, "Adaptive Fuzzy PI Controller for Speed of Separately Excited DC Motor," Kitakyusbu National College of Technology. 1999.

24. F. Tehrani, "A Control System for Oxygen Therapy Of Premature Infants," Proceedings of the 34rd Annual EMBS International Conference, 2001.

25. A. H. Noor, M. Kamal, and F. Yahya, "Application of PID Controller in Controlling Refrigerator Temperature," presented at the 5th IEEE International Colloquium on Signal Processing \& Its Application, 2009.

26. M.H. Moradi, "New Techniques for PID Controller Design," Department of Electrical Engineering, Iran, 2003.

27. R. Arulmozhiyal and R. Kandiban "Design of Fuzzy PID Controller for Brushless DC Motor," International Conference on Computer Communication and Informatics, Indiana, 2012.

28. J. Taube, R. Pillutla and J. Mills "Criteria For An Adaptive Fractional Inspired Oxygen Controller," Drexel University Phospho Engineering, Inc IEEE, 1988.

29. A. Arce, A. Real, and C. Bordons " Real - Time Implementation Of a Constrained MPC For Efficient Airflow Control In a PEM Fuel Cell," in IEEE Transaction on Industrial Electronics, 2010.

30. K. Bradely, and F. Roger, "Modeling and Control of Arterial Oxygen Saturation in Premature Infants," A thesis presented to the faculty of the Graduate school University of Missouri, 2011.

31. E.F. Camacho and C. Bordons, "Model Predictive Control” 1999, Springer. 
32. S. Skogestad, I. Postlethwaite, "Multivariable Feedback Control," John Wiley and Sons Ltd.: West Sussex, England. P.301-320, 2005

33. J.W. Severinghaus, "Simple Accurate Equations for Human Blood Oxygen Dissociation Computation,” J.Appl. Phys.:Resp. Environ. Exercise Physiol., 1979.

34. Y.Zhang, G.M. Cui, "Bias compensation models for stochastic systems with colored noise," Applied Mathematical Modeling, 2011.

35. Y. Zhang, "Unbiased identification of a class of multi-input single-output systems with correlated disturbances using bias compensation methods," Mathematical and computer Modeling, 2011.

36. F. Ding, Y.J. Liu, B, Bao, "Gradient based and least squares based iterative estimation algorithms for multi - input systems. Proceedings of the Institution of Mechanical Engineers,” Part I: Journal of Systems and Control Engineering, 2012.

37. M. Junxia, X. Weili, D. Rui, "Recursive relations of the criterion functions for the recursive least square algorithms," In IEEE, 2012.

38. P.E. Wellestead, and M.B. Zarrop, "Self - Tuning System: Control and signal processing,", Johau Wiley and Sous LTD, England 1991.

39. L. JI-Zhen, W. Yong, L. Xiang, "Modeling and Controller Design of Superheated Steam Temperatures System Based on SVM Combining Adaptive DMC,” IEEE 2007. 


\section{VITA}

Lafta Alkurawy was born in December, 1967 in the city of Baghdad, Iraq. He developed a genuine interest in engineering in high school, and chose Control and Computer Engineering as his major at the University of Technology in Baghdad, one of the best engineering schools in Iraq. He earned his bachelor's degree in Control and Computer Engineering from the same of the university. He earned his Master's degree in Control Engineering in Electrical and Computer Engineering from the same of the university in 2003. In August 2009, Mr. Lafta Alkurawy moved to the United States and pursued advanced education at the University of Missouri - Columbia. The following fall, Mr. Lafta Alkurawy started his doctoral program in the Department of Electrical and Computer Engineering at the University of Missouri in Columbia. 